\title{
Acoustic noise reduction methods for the launch pad
}

\author{
April 2020
}

\author{
Author: $\quad$ Iván Herrero Durá \\ Supervisors: Rubén Picó Vila \\ Vicente Romero García \\ Luis Miguel García Raffi \\ Víctor José Sánchez Morcillo
}





\section{Acknowledgements}

This work wouldn't have been possible without the help of many people that have been involved, either collaborating in the work or supporting me during this period.

First, I would like to thank my supervisors Rubén Picó, Vicente Romero, Lluís García and Víctor Sánchez for their guidance and constant support. Their vision and enthusiasm showed me the path during all the process of my $\mathrm{PhD}$. I want to thank also Alejandro Cebrecos, whose contributions have also been a fundamental part in this work.

None of the work presented here could have been possible without the funding provided by the European Space Agency. In this institution, I want to specially thank Julián Santiago (who sadly passed away in December 2018) and Ivan Ngan, both from the Structures, Mechanisms and Materials Division, for their help to make this project possible.

I want to thank also my colleagues and friends from the Brain Zone, who brought a lot of help but also fun along these years: Ahmed, Luis, María, Andrés, Dídac, Josep, Sergi, Susana, Jorge, Juan Carlos, Rober, Chiara, Vicent y Patri. Also to those persons that I found during the path: Maite, Jesús, Sara, Gema, Julien. Thanks to all of you.

The acknowledgment is also extended to my friends in Gandia: Rodri, Toni, Borja, Lorena, Aitor, Pamela and Ximo. 
Por supuesto, quiero agradecer a mi madre y mi hermana su apoyo incondicional y constante durante todos estos años. Vosotras habéis estado siempre ahí para compartir los momentos felices y para ayudarme y darme ánimos siempre que se ha presentado alguna dificultad, así que parte de este trabajo también es vuestro. 


\section{Abstract}

The sound pressure levels experienced by space vehicles during the initial stages of launch (engine ignition and lift-off) are extremely high and can significantly affect light transported structures, such as solar panels and antennas. The intense sound generated by the primary sources, the engine and the jet, is reinforced by the reflection at the bottom of the gas evacuation channel, which acts as a mirror from the acoustic point of view, and returns the energy released directly to the rocket and the structures it carries. This large amount of energy can put some launch missions at risk, with the economic and security consequences that this entails. Despite the relevance of this problem, knowledge about the characteristics of the sources, the behavior of ground facilities in reference to the dispersion, diffusion and absorption of sound, and the possible measures to mitigate the impact is still scarce. In this context, the main objective of this thesis will be developed. The purpose of this work is the design and optimization of a prototype system based on an array of Helmholtz resonators to maximize the absorption and dispersion of sound at ground level and, in this way, mitigate the sound pressure levels generated in these events in the aerospace context. The work of this thesis has been carried out within the framework of the Networking/Partnership Initiative contract 441-2015 of the European Space Agency.

First, a bibliographic study of the state of the art is carried out, focusing on the understanding of the characteristics of the acoustic environment on the launch pad, as well as its surroundings. Aspects such as noise sources and their characteristics, existing methods for noise mitigation (mainly based on the use of water as a cooling and absorbent agent) and the fundamentals of perfect 
sound absorption are included. Likewise, two studies previously developed in the context of space rocket noise mitigation, of special relevance for this work, are presented in detail. The first one is dedicated to the study of the behavior of the launching base from the acoustic point of view by building a scale model. In the second work we study the properties of sonic crystals as diffusion elements of acoustic waves, as well as their possible application for the mitigation of the acoustic field at the launch pad.

The theoretical foundations of the methodology used, based on the Transfer Matrix Method (TMM), are detailed below for problems in reflection and transmission. This method is applied in this work for the analysis of the critical coupling condition, through which it is possible to achieve perfect sound absorption by balancing radiation energy leaks in the system and its intrinsic losses. Based on this knowledge, the design of the Helmholtz resonator system for sound absorption and dispersion will be carried out.

In an introductory way, the evidence of the critical coupling is shown in an analytical, numerical and experimental form for the perfect absorption of sound in a system formed by a single Helmholtz resonator inserted in a waveguide. For this, an optimization algorithm based on the TMM is used to obtain the geometric parameters of a Helmholtz resonator so that it presents perfect sound absorption at the resonant frequency. The result of this optimization process is validated numerically using a simulation developed with the Finite Element Method (FEM) and experimentally.

This same design and analysis procedure is then used to design a system with quasi-perfect absorption in a frequency band. The system is composed of five Helmholtz resonators in a waveguide separated from each other by cavities. By means of the parameterization of the geometry of the resonators and the cavities, the system is optimized to achieve maximum energy absorption in a frequency range between $400 \mathrm{~Hz}$ and $560 \mathrm{~Hz}$. The resulting system presents the resonances of Helmholtz resonators, so as of the coupled modes between the resonant elements and the air cavities. The control of the losses of these modes allows maximizing the acoustic absorption coefficient in the desired frequency range. It is important to highlight that, in this first design, the characterization of the sound absorption properties is carried out only, without taking into account those of acoustic wave dispersion.

For the measurement of the sound absorption of the system, the Transfer Function Method (TFM) is used in an impedance tube, in a range of frequencies and amplitudes for which the plane incident wave propagation in linear regime can be considered. After the study, a good agreement between the analytical and 
experimental results is observed, having obtained a similar sound absorption coefficient in both magnitude and frequency range with the two methods.

Finally, an analysis of the system in free field conditions is proposed. This variation consists in replicating the Helmholtz resonator system in height, resulting in a periodic structure in one dimension. This replication has a double objective: on the one hand, it allows the creation of a periodic structure and, on the other, it reduces the system's edge effects. In this configuration, the system is not inserted in a waveguide and, therefore, perfectly plane incident waves cannot be assumed. Also, edge effects have an important role in the resulting acoustic field in front of the system, so they must be taken into account. Since the geometry and distribution of the resonant structures is the same, their properties in terms of sound absorption are also maintained. At the same time, this periodic distribution in height allows to study the system also from the point of view of the dispersion of acoustic waves. The behavior of this system in terms of these two physical phenomena has been characterized in free field in an anechoic chamber. A good agreement between the numerical model and the experimental results has been found, where a sound absorption coefficient, similar to the previous case, has been obtained both in magnitude and frequency range. 



\section{Resumen}

Los niveles de presión acústica experimentados por los vehículos espaciales durante las fases iniciales del lanzamiento (ignición de motores y despegue) son extremadamente elevados y pueden afectar significativamente a estructuras ligeras transportadas, como paneles solares y antenas. El intenso sonido generado por las fuentes primarias, el motor y el chorro, se ve reforzado por la reflexión en el fondo del canal de evacuación de gases, que actúa como un espejo desde el punto de vista acústico, y devuelve la energía liberada directamente al cohete y a las estructuras que transporta. Esta gran cantidad de energía puede poner en riesgo algunas misiones de lanzamiento, con las consecuencias económicas y de seguridad que ello conlleva. A pesar de la relevancia de este problema, el conocimiento sobre las características de las fuentes, el comportamiento de las instalaciones de suelo en referencia a la dispersión, difusión y absorción del sonido, y las posibles medidas para mitigar el impacto es todavía escaso. En este contexto se desarrollará el objetivo principal de esta Tesis, cuyo propósito es el diseño y optimización de un prototipo de sistema basado en un array de resonadores de Helmholtz para maximizar la absorción y dispersión del sonido a nivel del suelo y, de esta manera, mitigar los niveles de presión sonora generados en estos eventos en el contexto aeroespacial. Los trabajos de esta Tesis se han llevado a cabo en el marco del contrato Networking/Partnership Initiative 441-2015 de la Agencia Espacial Europea.

En primer lugar, se realiza un estudio bibliográfico del estado del arte centrado en la comprensión de las características del ambiente acústico en la plataforma de lanzamiento, así como en sus cercanías. Se incluyen aspectos tales como las fuentes de ruido y sus características, métodos existentes para la mitigación 
del ruido (principalmente basados en el uso de agua como agente refrigerador y absorbente) y los fundamentos de la absorción perfecta del sonido. Asimismo, se presentan en detalle dos estudios desarrollados previamente en el contexto de la mitigación del ruido de cohetes espaciales, de especial relevancia para este trabajo. El primero de ellos se dedica al estudio del comportamiento de la base de lanzamiento desde el punto de vista acústico mediante la construcción de una maqueta a escala. En el segundo trabajo se estudian las propiedades de los cristales de sonido como elementos difusores de ondas acústicas, así como su posible aplicación para la mitigación del campo acústico en la base de lanzamiento.

Los fundamentos teóricos de la metodología empleada, basada en el Método de Matrices de Transferencia (TMM), se detallan a continuación para problemas en reflexión y en transmisión. Este método se aplica en este trabajo para el análisis de la condición de acoplamiento crítico, a través del cual es posible conseguir absorción perfecta de sonido mediante el balance de las fugas de energía por radiación en el sistema y las pérdidas intrínsecas del mismo. En base a estos conocimientos se realizará el diseño del sistema de resonadores de Helmholtz para la absorción y dispersión del sonido.

A modo introductorio se muestra la evidencia del acoplamiento crítico de forma analítica, numérica y experimental para la absorción perfecta de sonido en un sistema formado por un único resonador de Helmholtz insertado en una guía de ondas. Para ello se emplea un algoritmo de optimización basado en el TMM para obtener los parámetros geométricos de un resonador de Helmholtz de tal forma que presente absorción perfecta de sonido a la frecuencia de resonancia. El resultado de este proceso de optimización se valida numéricamente utilizando una simulación desarrollada con el Método de Elementos Finitos (FEM) y de forma experimental.

Este mismo procedimiento de diseño y análisis se emplea a continuación para diseñar un sistema con absorción cuasi-perfecta en una banda de frecuencias. El sistema está compuesto por cinco resonadores de Helmholtz en una guía de ondas separados entre sí por cavidades. Mediante la parametrización de la geometría de los resonadores y las cavidades se consigue optimizar el sistema para conseguir la máxima absorción de energía en un rango de frecuencias comprendido entre $400 \mathrm{~Hz}$ y $560 \mathrm{~Hz}$. El sistema resultante presenta las resonancias propias de resonadores de Helmholtz, así como de los modos acoplados entre los elementos resonantes y las cavidades de aire. El control de las pérdidas de estos modos permite maximizar el coeficiente de absorción acústico en el rango de frecuencias deseado. Es importante destacar que, en este primer diseño se 
realiza la caracterización únicamente de sus propiedades de absorción sonora, sin tener en cuenta las de dispersión de ondas acústicas.

Para la medida de la absorción del sonido del sistema se emplea el Método de la Función de Transferencia (TFM) en un tubo de impedancia, en un rango de frecuencias y amplitudes para el cual se considera que la propagación de ondas es plana y en régimen lineal. Tras el estudio se observa un buen acuerdo entre los resultados analíticos y experimentales, habiéndose obtenido un coeficiente de absorción sonora similar tanto en magnitud como en rango de frecuencias con ambos métodos.

Finalmente, se propone estudiar el sistema en campo libre. Esta variación consiste en replicar el sistema de resonadores de Helmholtz en altura, dando como resultado una estructura periódica en una dimensión. Esta replicación tiene un doble objetivo: por un lado, permite crear una estructura periódica y, por otro, reduce los efectos de borde del sistema. En esta configuración, el sistema no se encuentra insertado en una guía de ondas y, por tanto, no se pueden asumir ondas incidentes perfectamente planas. Asimismo, los efectos de borde tienen un papel importante en el campo acústico resultante delante del sistema, por lo que deben ser tenidos en cuenta. Dado que la geometría y distribución de las estructuras resonantes es la misma, sus propiedades en cuanto a la absorción del sonido también se mantienen. Al mismo tiempo, esta distribución periódica en altura permite estudiar el sistema también desde el punto de vista de la dispersión de ondas acústicas. El comportamiento de este sistema en cuanto a estos dos fenómenos físicos se ha caracterizado en campo libre en una cámara anecoica. En el estudio de este sistema se ha encontrado también un buen ajuste entre el modelo numérico y los resultados experimentales, habiéndose obtenido, como en el caso anterior, un coeficiente de absorción sonora similar tanto en magnitud como en rango de frecuencias. 



\section{Resum}

Els nivells de pressió acústica experimentats pels vehicles espacials durant les fases inicials del llançament (ignició de motors i enlairament) són extremadament elevats i poden afectar significativament a estructures lleugeres transportades, com panells solars i antenes. L'intens soroll generat per les fonts primàries, el motor i el raig, es veu reforçat per la reflexió en el fons del canal d'evacuació de gasos, que actua com un mirall des del punt de vista acústic, i retorna l'energia alliberada directament al coet i a les estructures que transporta. Aquesta gran quantitat d'energia pot posar en risc algunes missions de llançament, amb les conseqüències econòmiques i de seguretat que això comporta. Tot i la rellevància d'aquest problema, el coneixement sobre les característiques de les fonts, el comportament de les instal-lacions de sòl en referència a la dispersió, difusió i absorció del so, i les possibles mesures per mitigar l'impacte és encara escàs. En aquest context es desenvoluparà l'objectiu principal d'aquesta Tesi, el propòsit de la qual és el disseny i optimització d'un prototip de sistema basat en una matriu de ressonadors de Helmholtz per maximitzar l'absorció i dispersió del so a nivell de terra i, d'aquesta manera, mitigar els nivells de pressió sonora generats en aquests esdeveniments en el context aeroespacial. Els treballs d'aquesta Tesi s'han dut a terme en el marc del contracte Networking/Partnership Initiative 441-2015 de l'Agència Espacial Europea.

En primer lloc, es realitza un estudi bibliogràfic de l'estat de l'art centrat en la comprensió de les característiques de l'ambient acústic a la plataforma de llançament, així com en les seves rodalies. S'inclouen aspectes com ara les fonts de soroll i les seves característiques, mètodes existents per a la mitigació 
de soroll (principalment basats en l'ús d'aigua com a agent refrigerador i absorbent) i els fonaments de l'absorció perfecta del so. Així mateix, es presenten en detall dos estudis desenvolupats prèviament en el context de la mitigació del soroll de coets espacials, d'especial rellevància per a aquest treball. El primer d'ells es dedica a l'estudi del comportament de la base de llançament des del punt de vista acústic mitjançant la construcció d'una maqueta a escala. En el segon treball s'estudien les propietats dels cristalls de so com a elements difusors d'ones acústiques, així com la seva possible aplicació per a la mitigació de el camp acústic a la base de llançament.

Els fonaments teòrics de la metodologia emprada, basada en el Mètode de Matrius de Transferència (TMM), es detallen a continuació per a problemes en reflexió i en transmissió. Aquest mètode s'aplica en aquest treball per a l'anàlisi de la condició d'acoblament crític, a través del qual és possible aconseguir absorció perfecta de so mitjançant el balanç de les fuites d'energia per radiació en el sistema i les pèrdues intrínseques del mateix. En base a aquests coneixements es realitzarà el disseny de sistema de ressonadors de Helmholtz per a l'absorció i dispersió del so.

A mode introductori es mostra l'evidència de l'acoblament crític de forma analítica, numèrica i experimental per a l'absorció perfecta de so en un sistema format per un únic ressonador de Helmholtz inserit en una guia d'ones. Per a això s'empra un algoritme d'optimització basat en el TMM per obtenir els paràmetres geomètrics d'un ressonador de Helmholtz de tal manera que presenti absorció perfecta de so a la freqüència de ressonància. El resultat d'aquest procés d'optimització es valida numèricament mitjançant una simulació desenvolupada amb el Mètode d'Elements Finits (FEM) i de forma experimental.

Aquest mateix procediment de disseny i anàlisi s'empra a continuació per dissenyar un sistema amb absorció quasi-perfecta en una banda de freqüències. El sistema està compost per cinc ressonadors de Helmholtz en una guia d'ones separats entre si per cavitats. Mitjançant la parametrització de la geometria dels ressonadors i les cavitats s'aconsegueix optimitzar el sistema per aconseguir la màxima absorció d'energia en un rang de freqüències comprès entre $400 \mathrm{~Hz}$ i $560 \mathrm{~Hz}$. El sistema resultant presenta les ressonàncies pròpies de ressonadors de Helmholtz, així com dels modes acoblats entre els elements ressonants i les cavitats d'aire. El control de les pèrdues d'aquestes maneres permet maximitzar el coeficient d'absorció acústic en el rang de freqüències desitjat. És important destacar que, en aquest primer disseny es realitza la caracterització únicament de les seves propietats d'absorció sonora, sense tenir en compte les de dispersió d'ones acústiques. 
Per a la mesura de l'absorció del so de sistema s'empra el Mètode de la Funció de Transferència (TFM) en un tub d'impedància, en un rang de freqüències i amplituds per al qual es considera que la propagació d'ones és plana i en règim lineal. En aquest estudi s'observa un bon acord entre els resultats analítics i experimentals, havent-se obtingut un coeficient d'absorció sonora similar tant en magnitud com en rang de freqüències amb els dos mètodes.

Finalment, es proposa estudiar el sistema en camp lliure. Aquesta variació consisteix a replicar el sistema de ressonadors de Helmholtz en alçada, donant com a resultat una estructura periòdica en una dimensió. Aquesta replicació té un doble objectiu: d'una banda, permet crear una estructura periòdica i, de l'altra, redueix els efectes de vora de el sistema. En aquesta configuració, el sistema no es troba inserit en una guia d'ones i, per tant, no es poden assumir ones incidents perfectament planes. Així mateix, els efectes de vora tenen un paper important en el camp acústic resultant davant de sistema, de manera que s'han de tenir en compte. Atès que la geometria i distribució de les estructures ressonants és la mateixa, les seves propietats pel que fa a l'absorció del so també es mantenen. Al mateix temps, aquesta distribució periòdica en alçada permet estudiar el sistema també des del punt de vista de la dispersió d'ones acústiques. El comportament d'aquest sistema pel que fa a aquests dos fenòmens físics s'ha caracteritzat en camp lliure en una cambra anecoica. En l'estudi d'aquest sistema s'ha trobat també un bon ajust entre el model numèric i els resultats experimentals, havent-se obtingut, com en el cas anterior, un coeficient d'absorció sonora similar tant en magnitud com en rang de freqüències. 



\section{Contents}

Abstract

Contents

xvii

1 State of the art 1

1.1 Acoustic environment of the launch pad: noise sources . . . . . . . . . 3

1.2 Methods for noise mitigation at the launch pad . . . . . . . . . 10

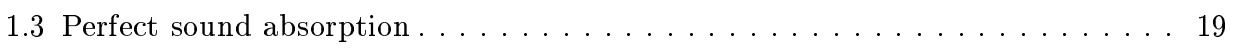

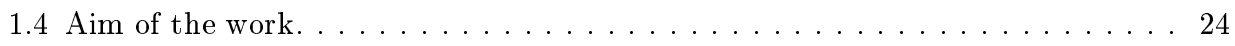

2 Preliminary results $\quad 27$

2.1 Study of the acoustic environment of the VEGA launch pad . . . . . . . . 27

2.2 Sonic crystals for noise reduction at the launch $p a d \ldots \ldots \ldots \ldots \ldots$ 
3 Transfer Matrix Method and critical coupling condition for acoustic waves

3.1 The Transfer Matrix Method . . . . . . . . . . . . . . . . . 46

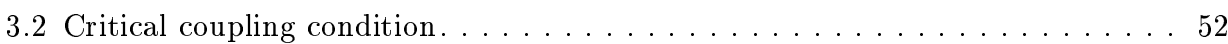

3.3 Problem in transmission: Critical coupling condition in thin aluminum plates . 53

3.4 Problem in reflection: Critical coupling condition in Helmholtz resonators . . . 66

3.5 Conclusions. . . . . . . . . . . . . . . . . . . . . . . . . . . 72

4 Experimental evidence of critical coupling condition for perfect absorption for a single frequency with Helmholtz resonators $\quad 75$

4.1 Design and optimization of perfect absorbers $\ldots \ldots \ldots \ldots \ldots$

4.2 Numerical model . . . . . . . . . . . . . . . . . . . 77

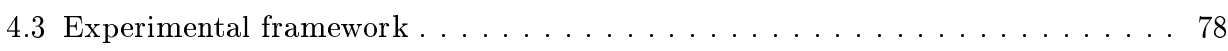

4.4 Results. . . . . . . . . . . . . . . . . . . . 81

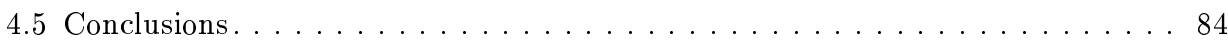

5 Design of a finite distribution of Helmholtz resonators for broadband absorption $\quad 85$

5.1 The Transfer Matrix Method for a multilayer system . . . . . . . . . 87

5.2 Insertion Loss in Reflection . . . . . . . . . . . . . . . . . . 87

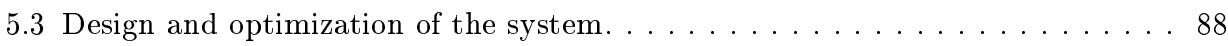

5.4 Numerical analysis methods. . . . . . . . . . . . . . . . . . 92

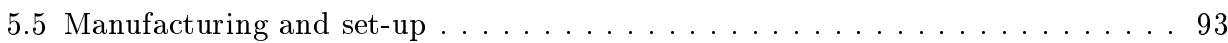

5.6 Results and discussion $\ldots \ldots \ldots \ldots \ldots \ldots \ldots \ldots$

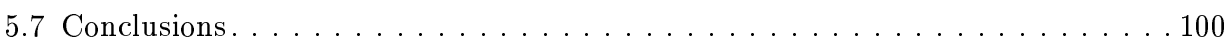

6 Design of a bi-dimensional distribution of Helmholtz resonators for broadband absorption 101

6.1 Parametric study of finite size effects. . . . . . . . . . . . . . . 103

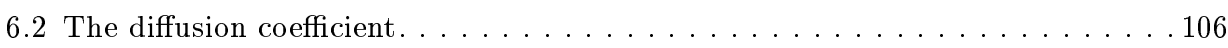

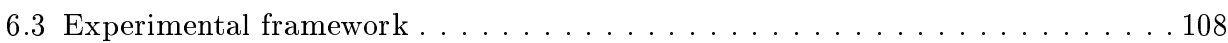

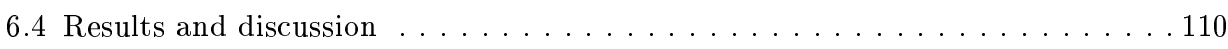




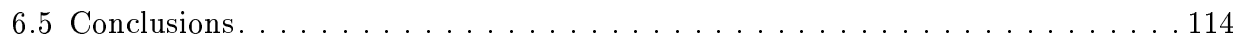

7 Conclusions and future work 117

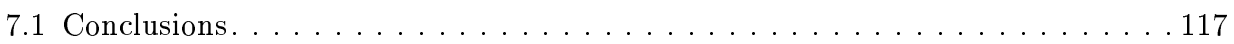

7.2 Future work . . . . . . . . . . . . . . . . . . . . 119

$\begin{array}{ll}\text { Bibliography } & 123\end{array}$ 



\section{Chapter 1}

\section{State of the art}

The launching of spacecrafts is an extreme event, where the physical magnitudes involved (temperature, gas flow, pressure) exceed those on conventional situations by several orders of magnitude [1]. From the point of view of acoustics, they generate the highest noise level produced by humans, reaching a sound pressure level up to $180 \mathrm{~dB}$. As a reference, it is important to note that the pain threshold in humans is $130 \mathrm{~dB}$.

Launch pads are the above-ground facilities from which spacecrafts are vertically launched. Traditionally, these facilities have been designed paying special attention to certain aspects related to aerodynamics criteria and gas flow evacuation, among others.

These facilities are usually made of concrete, a rigid and resistant material able to withstand high temperatures. The shape of the launch pads (a general scheme of a launch pad is shown in Figure 1.1) is designed to facilitate the evacuation of the gas flow produced by the engine and the exhaust of the rocket during the ignition and first stages of the lift-off. For this reason, inverted Vshape elements, named flame deflectors, are included.

This design ensures the correct functioning of the facility regarding to the gas flow. Nevertheless, a huge amount of energy is released also in form of acoustic waves, giving as a result an enormous acoustic load on the rocket and the payload (generally satellites and sensitive instrumentation, either for a scientific, military or commercial use). Every part of a launch vehicle, a launch pad, 


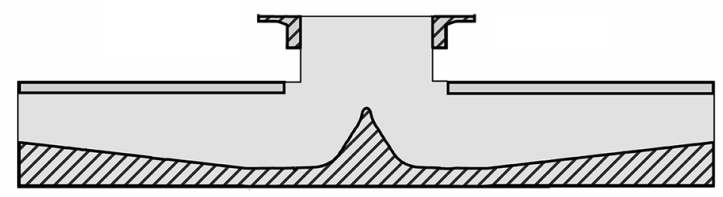

Figure 1.1: 2D scheme of a longitudinal section of the launch pad.

and the ground operation equipment is then subject to the high acoustic load generated during lift-off [2]. Shock waves, whose acoustic intensity depends primarily on both the size of the rocket, and its exhaust velocity, are formed by the collision of the supersonic exhaust gases with the ambient air [3]. In this situation, the bottom of the launch pad acts as a reflecting surface that sends the acoustic waves back, giving as a result vibrations that may have severe effects on these elements. Damage usually occurs in the secondary structure of the aircraft as a result of a large number of relatively small loads applied at the rate of several hundred loading cycles per second [4].

Potential problems which may result from acoustic loading include [5]:

- Malfunction of electronic and mechanical components in the vehicle (from structural vibration and internal acoustic loading).

- Fatigue failure of internal components and supporting hardware, such as cable-bundle supports, instrument-mounting brackets, and distributed piping systems (from structural vibration).

- Fatigue of lightweight exterior structures, such as aerodynamic fins and antenna panels (from direct external acoustic loading).

- Fatigue of lightweight spacecraft structures (from internal acoustic noise and structural vibration).

- Adverse environmental conditions for vehicle occupant.

The effect is so dramatic that it can put the missions into risk and has considerable economical consequences. Despite this fact, there is still little knowledge on the characteristics of the sources and the behavior of the ground facilities concerning sound scattering, diffusion and absorption. For this reason, the acoustic load affecting the structures needs to be characterized and reduced as much as possible in order to ease its correct functioning. 
As the payload becomes more and more sensitive, new needs concerning noise reduction appear and, as a consequence, research is being developed in this field with the purpose of finding a solution that can be applied to the mitigation of the acoustic load to which the rocket is subject. Due to the lack of theoretical approaches to predict the supersonic jet noise levels with the required accuracy [6] and for economic profitability reasons, these studies are usually performed at smaller scales and the obtained results are extrapolated to the real case in those cases in which the magnitudes studied are scalable.

\subsection{Acoustic environment of the launch pad: noise sources}

The first step towards the evaluation of any noise mitigation process is to identify the acoustic sources [2]. The characterization of the acoustic field of the launch pad is an important aim in order to both understand the phenomena taking place and optimize solutions. These measurements are done, in most cases, by using a microphone phased-array in order to locate the directions of the incident acoustic waves. In this field, some studies have been performed concerning the acoustic sources of the Ariane 5 rocket [7] and the VEGA launch vehicle [8] at lift-off, both rockets belong to the European Space Agency (ESA). VEGA is devoted to launch and set into orbit of small payloads (such as satellites for scientific missions), and Ariane 5 has a much higher lift capacity. The typical launch configuration used in current rocket launch can be seen in Figure 1.2.

The problem of high acoustic load in the vicinity of the launch pad is becoming important for space agencies. As a consequence, these institutions are developing new programs for the study of the acoustic environment of the launch pad and the noise sources that contribute to it.

It is important to note that jet noise during the lift-off of a launch vehicle is complicated to characterize due to the simultaneous flow of multiple jets, and their deflection by jet deflectors. For supersonic transports and military aircrafts the exhaust jet velocities are supersonic [10]. In these type of jets, the exhaust velocity is higher than the speed of sound in the corresponding surrounding medium (in the case of air at a temperature of $20^{\circ} \mathrm{C}, c \simeq 340$ $\mathrm{m} / \mathrm{s})$. 


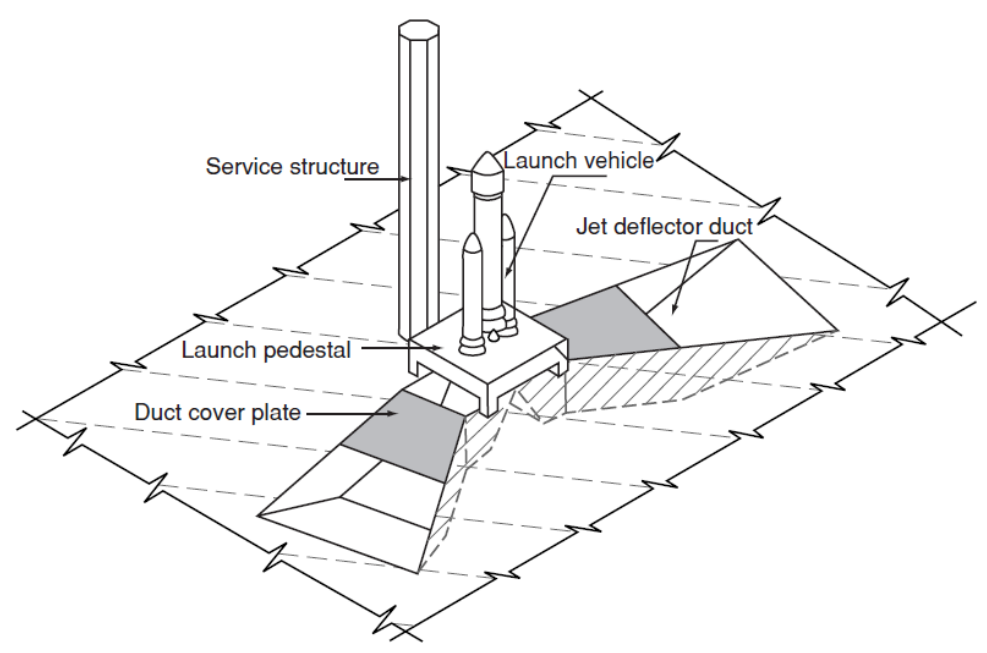

Figure 1.2: Typical launch configuration (from [9]).

\subsubsection{Characteristics of the noise sources}

The acoustic sources were classified in two types in the study developed by Tsutsumi et al. on the advanced solid rocket in the Japanese Aerospace Exploration Agency (JAXA) [11]: i) "impinging noise" due to the impingement of the exhaust plume to the flame deflector, and $i i$ ) the Mach wave due to the unsteadiness of the supersonic plume flowing along the flame deflectors. The Mach wave propagates away from the vehicle, so that this effect is relatively weak. However, the impinging noise propagates directly to the vehicle, so that it is a dominant noise source.

The primary source of noise is the noise of the two jets emerging from the base of the launch vehicle [9]. Supersonic jet noise consists of three main components: turbulent mixing noise, comprising the contribution of large-scale and fine-scale structures; broadband shock noise; and screech tones [12, 13]. These components are present in all the type of jets except for jets operating at perfectly expanded conditions (in which the exit pressure is exactly equal to the ambient pressure) [14]. The typical noise spectrum of imperfectly expanded jets was measured by Seiner in 1984 [15] (see Figure 1.3). The first category is present in both subsonic and supersonic jets but can have different mechanisms, while the other two occur only in supersonic jets [16].

An exhaustive study about the three basic components of the noise of a supersonic jet has been done by Tam et al. [14]. The authors concluded that the 


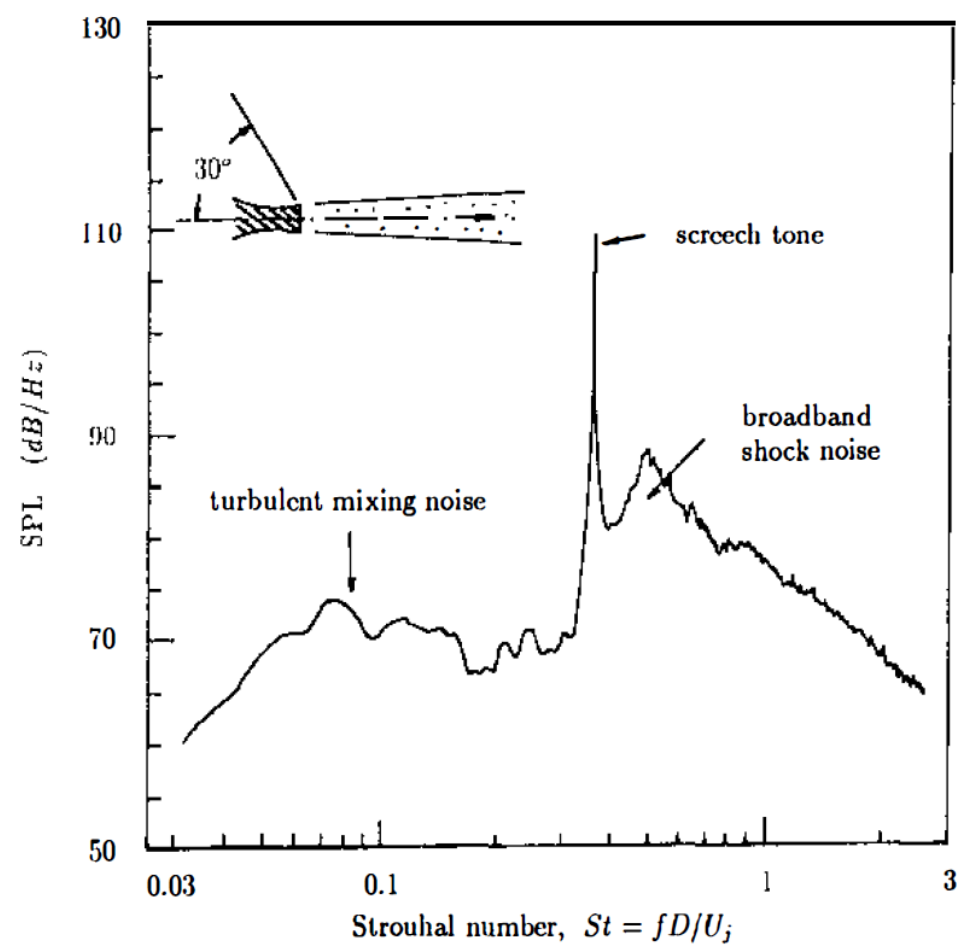

Figure 1.3: Typical narrow-band noise spectrum of an imperfectly expanded jet (from [15]). In this graph $f, D$ and $U_{i}$ correspond to the frequency, the nozzle exit diameter and the fully expanded jet velocity, respectively. $S P L$ is the sound pressure level. 
turbulent mixing noise is radiated into an angular sector of about 45 to 60 degrees, measured from the jet flow direction. At the same time, they identified the following characteristics in the broadband shock-associated noise:

- The frequency corresponding to the peak of the spectrum changes with the direction of radiation (it increases with the inlet angle).

- The spectrum presents several peaks.

- The half-width of the peak in Figure 1.3 corresponding to this type of noise increases with the inlet angle.

Norum [17] found that the fundamental screech tone of each mode attains a maximum amplitude at about $20^{\circ}$ from the inlet axis, with higher harmonics exhibiting multiple lobes.

Based on the Ariane 5 rocket at lift-off, Elias [7] performed a study by placing an acoustic antenna formed by three circles of the radius of the fairing and equipped each with 4 microphones all the $90^{\circ}$ at $35 \mathrm{~m}$ above the central engine exhaust location. This study concluded that the sound field around the launcher is very complex because of the interaction of the jets with the launch pad. It is very important to note that the contribution of each source and the overall noise map is time dependent, as other parameters must be taken into account such as the height of the rocket in different phases of the lift-off. The highest levels were registered during tests executed at low altitudes (from 0 to $25 \mathrm{~m}$ ), while the lowest levels were measured with the test at the highest altitude. In the study developed in 2013 by the National Aeronautics and Space Administration (NASA) for the real scale NASA Wallops launch pad [18], the duct exit was found to be the primary source during static-test firing.

For the future launch pad of Ariane 6, foreseen to be finished in 2020, an experimental characterization was done by Malbéqui et al. [19] by means of a test campaign carried out at the Martel facility with a mock-up of the launch pad at $1 / 40$ scale. In the free field, the upstream radiation of the supersonic jet is described by the three components mentioned above. The turbulent mixing component mainly radiates downstream the jet. Nevertheless, when the launch pad is present, the configuration is more complex. At low altitudes of the launcher, typically from 0 to $30 D$ (where $D$ is the diameter of the nozzle's exit), the screech and tone noise still radiates upstream, while the three following sources can also contribute: i) the turbulent mixing noise emitted dowstream the jet reflecting on the launch table, ii) the acoustical waves radiated at the exit of the trench and iii) the additional sources generated by the impacts 


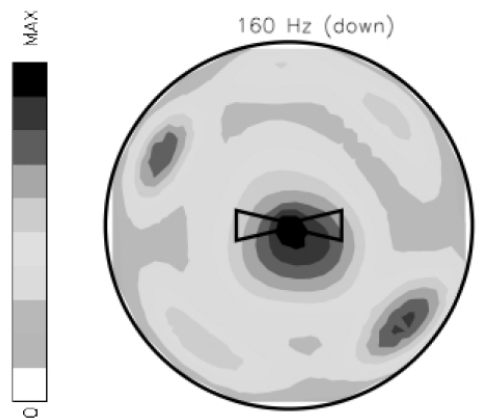

Figure 1.4: Acoustic sources localization obtained by classical beamforming (from [8]). The straight lines in the center of the figure represent the top view of the launch pad.

of the jet on the launch table. The maximum of the sound pressure level is reached when the height is approximately $20 \mathrm{D}$.

Based on the VEGA launch vehicle at lift-off, Gély [8] performed a study for acoustic sources localization by placing 26 microphones in the fairing area with the main objective of locating the directions of the incident acoustic plane waves reaching the fairing. In order to locate the radiating regions, the author describes the incident field as a set of uncorrelated plane waves. The results of the study are shown in Figure 1.4. The study concluded that the main source is the exhaust of the rocket, although some of the ground facilities, like the towers placed in the vicinity of the launch pad, have an important contribution to the sound pressure field.

The Ignition Over Pressure $(I O P)$ related to the launch vehicle lift-off has also been studied [20]. This term is defined as the pressure disturbance which originates from the rocket ignition process. These pressure disturbances are originated due to the interaction of the sound waves with the launch vehicle and its surroundings. Thus, the $I O P$ is resulting from fluid dynamic compression on the accelerating plume gas, subsequent rarefaction and propagation during the pressure rise rate period in the rocket combustion chamber. Results of this study indicate that sudden shock front is generated due to the shock wave. This shock front initially propagates at supersonic speed and later decays with time.

As a result of the studies cited in this Section, it is possible to conclude that the main source of noise during the first stages of the lift-off are the jets emerging from the launch vehicle and, in a more specific way, impinging noise due to the 
impingement of the exhaust plume to the flame deflector. The acoustic load in these events has been found to be higher at low altitudes, mainly from 0 to 25 $\mathrm{m}$. Additionally, the ground facilities placed in the vicinity of the spacecraft may have an important effect during the ignition and first stages of the lift-off.

\subsubsection{Modeling of the noise sources}

Different forms of modeling the acoustic sources have been discussed in the literature during the last years. In 1971, Eldred [5] developed an empirical model based on the observation that each slice of the jet contributes to acoustic radiation, mostly in the frequency band $f_{i}$ that depends on the distance of the slice from the exhaust [21]. Indeed, because the size of the turbulent structures increases as their distance from the exhaust increases, the acoustic peak frequency for each slice is a decreasing function of the slice distance from the exhaust. Some modifications of this model have been proposed since its first publication [22-24], being the most recent the one proposed by Barbarino et al. in 2017 [25]. In this last modification, the authors proposed a Boundary Elements Method (BEM) frequency-domain Computation Aero-Acoustics (CAA) method to propagate the noise generated by elementary sources distributed along the plume, using an uncorrelated superposition of these elementary fields, taking also into account how to control the correlation and giving a new formulation that contains an explicit expression for the acoustic pressure of each noise source in terms of amplitude and phase. For the study, the authors used data obtained from a 1/20 scaled mock-up of VEGA, conceived to reproduce the same acoustic field generated by the full-scale rocket motor P80 at different altitudes, from 0 to $75 \mathrm{~m}$, corresponding to the first 4 seconds of VEGA's ascent trajectory during lift-off. Flush-mounted microphones were installed along a circumference around each axial location (see Figure 1.5) and the noise spectra were measured. The results of this study showed that the uncorrelated model provides a better prediction than the correlated one at 0 and $10 \mathrm{~m}$ while the correlated one is better at $75 \mathrm{~m}$.

\subsubsection{Other elements affecting the acoustic environment}

The launch pad acts as a mirror for the acoustic sources creating source images and contributing to the distribution of the acoustic field and, thus, its geometry must be taken into account. As it has been already mentioned, two flame deflectors are included in the launch pad to ensure the correct gas flow evacuation. However, the presence of these deflectors can be counter-productive from the acoustic point of view in the initial stages of the launch due to the 


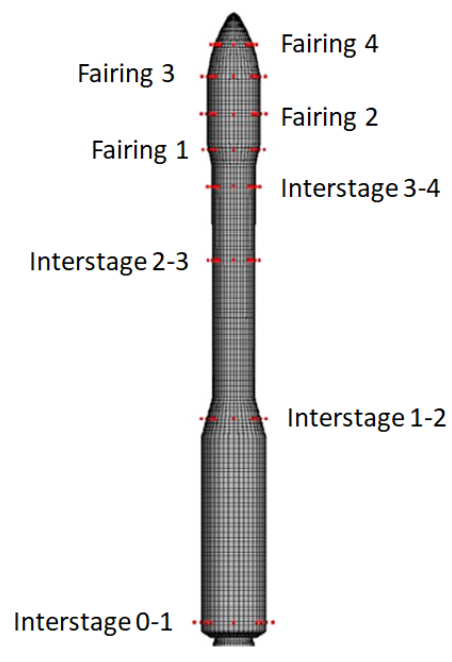

Figure 1.5: Microphone locations in the study developed by Barbarino et al. (from [25])

jet impinging on them. As a result, impinging noise propagates directly to the vehicle and it is a dominant noise source. A curved flame deflector with steep initial slope was found to be desirable for the lower acoustic level for the vehicle with cost-effectiveness by Tsutsumi et al. [11].

In a more general context, Eldred [5] published some statements related to aspects to be considered to minimize the acoustic loading:

- The angle through which the exhaust flow is deflected is kept as small as possible. This will normally require the vehicle to be set at some distance above the ground; otherwise, a deflection angle greater than 90 degrees will result with increased acoustic levels.

- The exhaust flow is deflected through a covered bucket or tunnel to shield the vehicle from direct radiation from the deflected flow.

- The deflector and test stand are designed to eliminate large reflective surfaces turned toward the space vehicle.

- Water is injected into the deflector near the nozzle. 
- Acoustical-attenuation padding is placed over sensitive areas of the vehicle.

The different elements contributing to the acoustic environment at the launch pad have been studied and presented here by means of a review of the state of the art. The main source affecting the acoustic environment is the supersonic jet generated by the spacecraft during the ignition and first stages of the liftoff, although the geometry of the launch pad is also important. The knowledge of these sources is crucial to find appropriate solutions to mitigate the acoustic load that the rocket has to bear. In the next section, some solutions that have been previously studied will be detailed.

\subsection{Methods for noise mitigation at the launch pad}

As it has been mentioned in the previous section, the extreme acoustic environment resulting from the ignition and first stages of the lift-off of spacecrafts can affect its correct operation. This fact became clear since the beginning of the launching of space vehicles, and the space agencies realized that substantial savings in unexpected repairs, operating costs and system failures could be realized by even relatively small reductions in the rocket launch noise level [3]. Passive solutions to mitigate the acoustic load are mostly used during the lift-off of spacecrafts, such as water deluge systems and, when the launch pad is being designed, its geometrical parameters.

\subsubsection{Water deluge systems}

The air, water and aqueous microjet injection are extensively used in the jet noise research area. Water, in particular, is widely used to reduce large pressure fluctuations occurring in supersonic jets, as well as the blast wave generated at the ignition of the solid propellant in the rocket motor of a space launcher [27]. The jet noise reduction can be achieved by modifying the parameters at the origin of the flow (diameter, coaxing flows) or by acting on the exhaust flow downstream of the nozzle exit (water injection) [28].

The most used strategy to reduce the noise pressure level is the injection of a hot supersonic pressurized water flow injected all around the pad [29]. There are two main effects of water injection on the jet leading to a decrease of acoustic levels: the reduction of both the jet velocity and the jet temperature [30]. The decrease of jet velocity is caused by momentum transfer between the liquid and the gaseous phases, and the reduction of the jet temperature is 


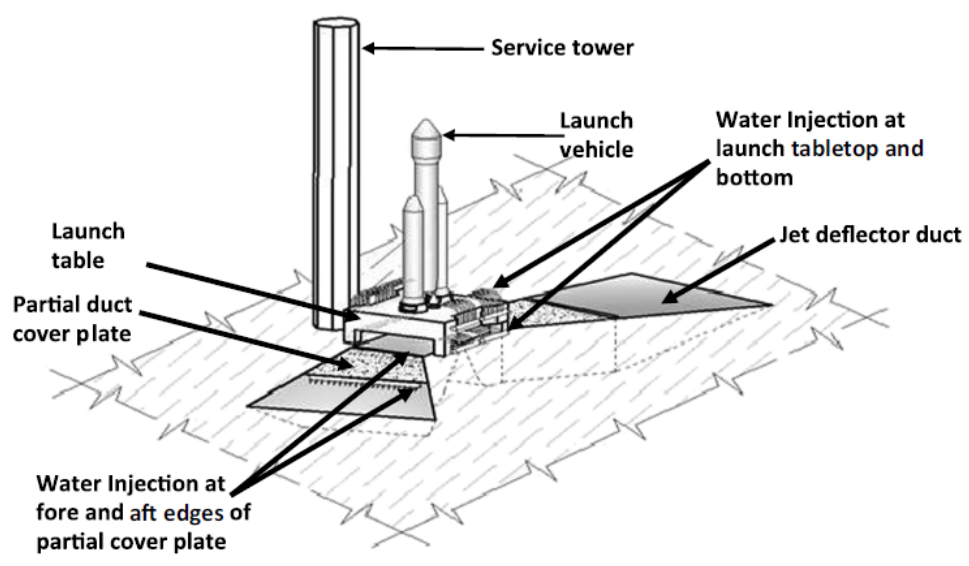

Figure 1.6: Scheme of the launch pad with water injection acoustic suppression system (from [6]).

achieved due to partial vaporization of the injected water [31]. Nevertheless, this strategy has an important drawback: water is often a degrading agent for materials and structures, creating corrosion or other damages if it is applied directly on the structures.

Analogously to Figure 1.2, in which the typical launch configuration used in current rocket launch can be observed, a proposal of the position for the water injection system is shown in Figure 1.6, where the nozzles are placed before and after the edges of the partial cover plate and at the launch tabletop and bottom.

In general, the sources of dissipation may be divided into two general categories: (i) those intrinsic to the medium and (ii) those associated with the boundaries of the medium [32]. Sound attenuation in fluids representing dissipation of acoustic energy from a sound wave, occurs through a number of physical processes involving molecular viscosity, thermal conductivity, and other dissipative or relaxation processes [33].

Viscous losses occur whenever there is a relative motion between adjacent portions of the medium. Heat conduction losses result from the conduction of thermal energy from higher temperature condensations to lower temperature rarefactions. Molecular processes leading to absorption include the conversion of the kinetic energy of the molecules into stored potential energy, rotational 
and vibrational energies, and energies of association between different ionic species and complexes in ionized solutions [32].

The effectiveness of the solution proposed for mitigation noise based on water deluge depends on several parameters $[6,34]$ :

- Position of the water system: injection closer to the nozzle exit leads to better reduction, mainly due to suppression in the high-frequency range when observed from downstream [35]. Water injection at different locations on the launch pad suppresses the noise emitted more effectively for vehicle locations earlier in the lift-off sequence than later [6].

- Temperature of the jet: comparing cold and hot high supersonic jets of the same exit velocity, elevated jet temperature causes decreases of the radiated noise due to the reduction in the Lighthill's stress tensor term [36]. The reduction is nearly independent of the jet temperature in the 600-900 K range [9].

- Number of injectors: it is found that a multipoint injection scheme is very effective in the launch scenario [36].

- Droplet mass concentration: there is a spectral broadening effect in the attenuation coefficient with a decrease in droplet mass concentration [37].

- Injection angle of the jet: injection angle of $60^{\circ}$ has greater effectiveness to reduce pressure pulsation levels [38, 39].

- Pressure and water flow: in general, the amount of noise reduction obtained increases both with mass flow and pressure of the injected water $[40,41]$.

The application of water injection into an altitude diffuser exhaust plume for noise reduction has been also recently studied [16]. Water injection has the potential to adversely affect the performance of the supersonic diffuser when water is injected in the diffuser exhaust plume at different axial locations.

The narrowband spectral measurements of the far-field noise made on an axisymmetric heated jet indicate that water injection reduces the sound pressure level at low frequencies without increasing the high-frequency noise [42]. The presence of water droplets in the jet leads to a significant modification of the turbulence in the structure (see Figure 1.7), reducing the velocity compared to that of a normal jet and achieving a far-field noise level reduction up to $6 \mathrm{~dB}$ due to the vaporization of liquid droplets. 

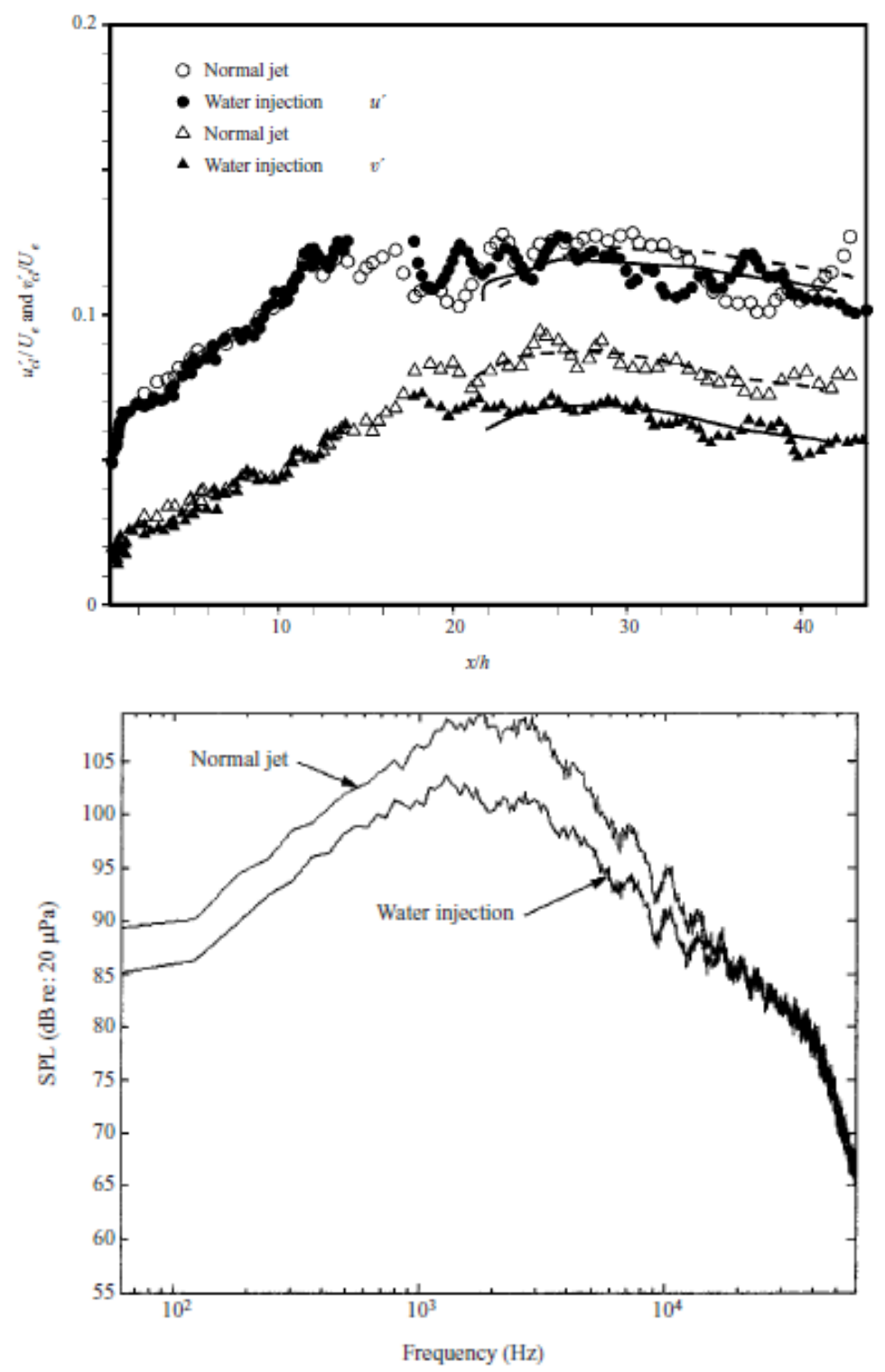

Figure 1.7: Variation of the normalized centreline turbulence intensities with downstream distance of the rectangular jet (top) and narrowband far-field spectrum of the axisymmetric jet at $\theta=140^{\circ}$ (bottom) (both figures from [42]). In this graph $u^{\prime}$ and $v^{\prime}$ are, respectively, the axial and transverse turbulence intensities, $U_{e}$ is the jet exit velocity, $x$ indicates the position at which the flow is considered and $h$ is the nozzle exit height. 
For the case of mid-frequencies the wavelength is approximately of the same order of magnitude of the size of the flow structure. In this regime, part of the energy is reflected back in a direction depending on the angle of incidence of the wave with respect to the water system; other part is diffracted and, as a consequence, the interaction wave/water leads to an energy spreading. The rest is absorbed by the water.

At high frequencies, where the wavelength is very short, it has been found that the nonlinear particle relaxation processes are primarily responsible for reduction in the linear absorption coefficient [33].

The study carried out by Zoppellari and Juvé [31] showed that injecting a large amount of water (several times the mass of the jet) into a supersonic jet results in a significant decrease in the far-field noise, up to $10 \mathrm{~dB}$. An ongoing investigation limits the amount of water injected to a small fraction of the total mass of the jet of approximately 0.1 [42].

Some works present aqueous microjet injection as a very effective method at reducing the far-field noise produced by high-speed jets, achieving reductions up to $6 \mathrm{~dB}$ of mixing noise and Mach wave radiation [38]. However, other works catalog aqueous injection for in-flight jet noise reduction using techniques investigated thus far as unrealistic claiming that, while water injection in laboratory experiments has produced reasonably high overall sound pressure level reductions, the injection pressures and mass flow requirements are impractical for aircraft applications [43].

In the frame of the VEGA program, a collaboration between AVIO and ONERA, Gély et al. [8] studied the effect of several parameters in the sound pressure level at the launch pad. The authors studied the effect of the number of flues, placing covers in them, launch vehicle altitude and the use of a water injection system, as well as the combination between them. A picture of the experimental set-up including the water injection system can be observed in Figure 1.8. The main results of the study were the following:

- Effect of the number of flues: there are no significant differences, in terms of $S P L$ measured between the configuration with single and double flues $(S P L<1 \mathrm{~dB})$.

- Effects of covering of the flues: maximum reduction of the $S P L$ levels of 1-2 $\mathrm{dB}$ in the low frequency range (up to $150 \mathrm{~Hz}$ ).

- Effects of water injection: maximum attenuation of the $S P L$ from 1 to $4 \mathrm{~dB}$, which is mostly effective in the low frequency region. 


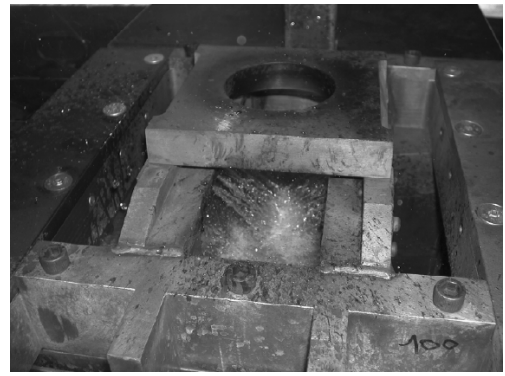

Figure 1.8: Water injection devices used in the experiment of Gély et al. (from [8]).
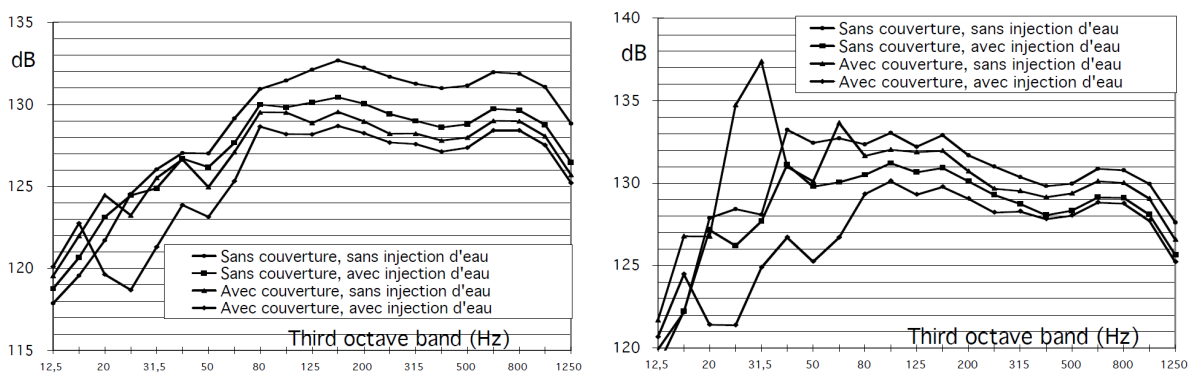

Figure 1.9: Flue cover and water injection effect in a single (left) and double (right) flues configuration (from [44]).

- Effects of launch vehicle altitude: the investigated altitudes correspond in full scale to $0 \mathrm{~m}, 10 \mathrm{~m}, 25 \mathrm{~m}$ and $50 \mathrm{~m}$. The results show an increase of the $S P L$ with quote up to $25 \mathrm{~m}$, followed by a strong reduction of the acoustic levels for higher altitudes.

- Effects of interaction cover + water injection: this combination produces a strong reduction (from $3 \mathrm{~dB}$ up to $10 \mathrm{~dB}$ ) of $S P L$ in all the octave bands.

- Effects of interaction cover + altitude: this combination gives the maximum efficiency al low altitudes (reductions up to $4 \mathrm{~dB}$ up to $25 \mathrm{~m}$ in full scale), becoming ineffective at higher altitudes.

- Effects of water injection + altitude: mostly efficient at low altitudes, with a maximum noise reduction at $0 \mathrm{~m}$ (of the order of $4 \mathrm{~dB}$ ). For altitudes above $25 \mathrm{~m}$ the effect of water injection is negligible. 

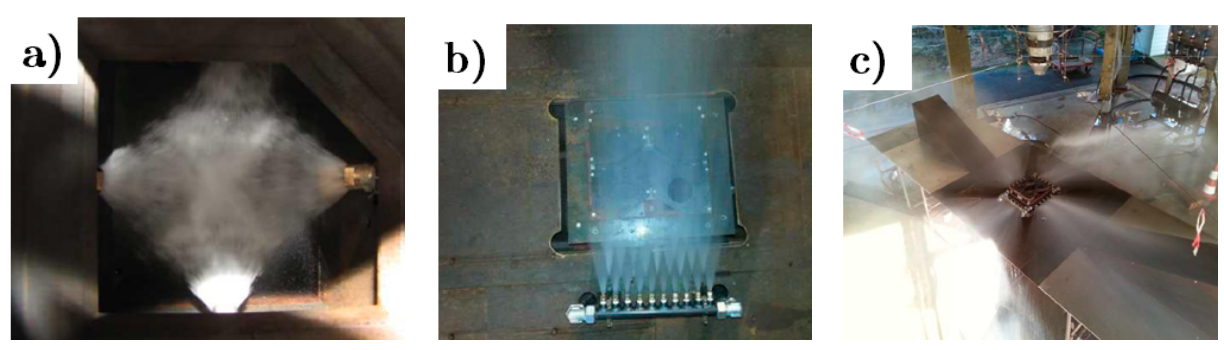

Figure 1.10: a) Water injection at the trench entrance, b) Water deluge above the launch table, c) Water deluge outwards the launch table (the three figures are from [19])

In order to reduce the radiated field, Malbéqui et al. [19] tested three different types of water injection system on the launch pad: the first one (Figure 1.10a)) was composed by 4 nozzles located at the entrance of the trench and inside it, the second one (Figure 1.10b)) was composed by a ramp of nozzles so that the water is injected above the launch table, and the third one (Figure 1.10c)) was made of 4 ramps of nozzles surrounding the launch table and debiting outwards. In this work, the authors found that the water deluge curtain outwards the table does not provide significant additional attenuation, while the first and the second configurations gave a significant level attenuation of about $6 \mathrm{~dB}$ and from 1 to $3 \mathrm{~dB}$, respectively.

\subsubsection{Solutions acting on the launch pad: flame deflectors and channels}

Passive noise control solutions refer to that kind of systems that allow reducing the sound pressure level in sensitive structures without the need to act on the rocket, but in its vicinity. These solutions imply acting on the launch pad, as it is a facility that highly affects the acoustic field arriving to the rocket and the payload. The geometry, components as flame deflectors and gas flow evacuation ducts, or height at which the rocket is placed during the first stages of the lift-off are of crucial importance to create the right acoustic environment conditions for the launch. However, these solutions have some limitations. The most important one is that changing the geometry of the existing launch pad is a very expensive solution and is economically non-viable, so these measures should be considered in the design of future launch pads.

Flame deflectors are an important part of launch pads, used to channel the exhaust gases away from the pad. The inclusion of these elements is nonoptional from the point of view of the evacuation of the gas flow generated by 

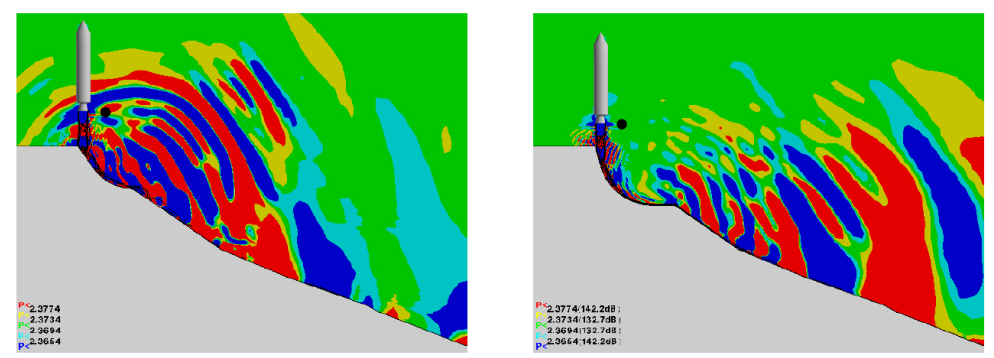

Figure 1.11: Comparison of non-dimensional static pressure distribution by considering two different tilt angles (from [11]).

Table 1.1: Overall sound pressure level (OASPL) for each studied type of flame deflectors (data extracted from [45]).

\begin{tabular}{|l|c|}
\hline Flame deflector & OASPL $[\mathbf{d B}]$ \\
\hline $45^{\circ}$ flat plate & 107 \\
\hline $45^{\circ}$ curved plate & 110 \\
\hline $90^{\circ}$ bucket & 117 \\
\hline $150^{\circ}$ bucket & 125 \\
\hline Normal flat plate & 104 \\
\hline Normal conical flat plate & 101 \\
\hline
\end{tabular}

the rocket and, therefore, the most sensible solution in this case is choosing a shape that can satisfy this need together with the mitigation of the acoustic field. In this area, a curved flame deflector with steep initial slope was found by Tsutsumi et al. [11], after studying different geometries, to be desirable for obtaining of low acoustic levels for the vehicle with cost-effectiveness. The effect of modifying the shape of the flame deflectors can be observed in Figure 1.11 .

In 2013, Madhan Kumar et al. [45] carried out a study in which deflectors of various shapes and sizes were considered, and performed an empirical analysis in various noise fields. The authors concluded that the deflector in which no covers are placed and, for instance, are all opened will have an increase of between 6 to $20 \mathrm{~dB}$ in the sound pressure level. In order to avoid sound reflection points, they enhanced the bucket-type deflectors to reduce overall sound pressure level $(O A S P L)$ and, for instance, the acoustic loading. The $O A S P L$ of each studied type of flame deflectors is detailed in Table 1.1. 


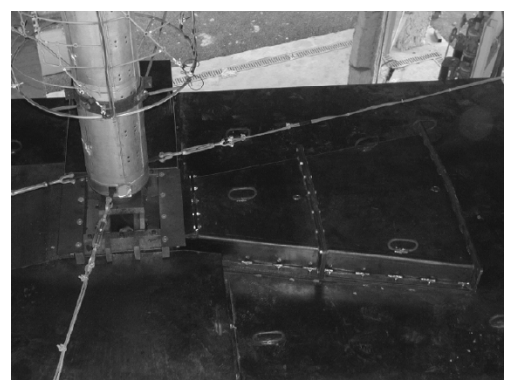

Figure 1.12: Flue covers (from [8]).

Also adding covers on the gas flow evacuation channels would reduce direct reflections towards the rocket and the payload and, at the same time, permits the gas flow circulation. The effect of covering the channel is sensitive for different angular positions, having important effects in the sound pressure level reduction at frequencies above $80 \mathrm{~Hz}$ [46].

As it was mentioned in the previous Section, the study of Gély et al. [8] considered the placement of covers of the channels (see Figure 1.12). The experimental measurements carried out in this work, show a maximum reduction of the SPL of 1-2 dB in the low frequency range (up to $150 \mathrm{~Hz}$ ), and a maximum efficiency at low altitudes (reductions up to $4 \mathrm{~dB}$ up to $25 \mathrm{~m}$ in full scale), becoming ineffective at high altitudes. Furthermore, the combination of the covers with a water injection system produces a strong reduction of the SPL from $3 \mathrm{~dB}$ up to $10 \mathrm{~dB}$.

In the study of Malbéqui et al., in which the characterization of the jet noise on the Ariane 6 launcher was done, the launch pad consists of two covered long trenches guiding both the jets generated by the solid rocket boosters and the main cryogenic engine. It was found, by using a microphone on the payload, that the covered trenches strongly attenuate the level at low altitudes.

\subsubsection{Solutions based on sonic crystals}

A sonic crystal (SC) consists of a periodic array of rigid elements or scatterers immersed in a host fluid medium [47] in which only longitudinal waves are allowed to propagate. This periodicity confers these structures some special properties with a particular interest in the control of acoustic waves.

The feasibility of the use of SCs in reducing the impact of the backward reflected field emitted by a sound source was studied in [48] and [49] in a sim- 
plified scaled model of a launch pad. A summary of the study and its main results and conclusions will be exposed in the next Chapter.

\subsection{Perfect sound absorption}

The methods that have been detailed in the previous Section for the mitigation of the acoustic load during the first stages of spacecrafts' lift-off are based on different mechanisms, such as modifying the direction to which the sound waves are reflected by the bottom of the launch pad by changing the shape or making use of the diffusive properties of sonic crystals. These methods have proven a certain effectiveness for achieving this goal. Nevertheless, other methods can be studied to be applied in these contexts, both individually or combined with the ones that are being used nowadays. A good example of this is the use of systems presenting perfect sound absorption.

Sound absorption has always been a trending topic in the field of Acoustics due to its interest for the control of noise and vibrations, insulation of buildings and many other applications. This topic has been extensively studied and widely exploited along decades. Nevertheless, traditional methods such as porous materials, micro-perforated plates and sound absorption wedges show severe limitations in this respect $[50,51]$, which make necessary the study of new methods to increase the efficiency and effectiveness. A highly efficient sound absorption device requires not only a proper mechanism of energy conversion but also an impedance matched surface to air, so that sound can efficiently enter the absorber [52].

One of the most recent topics in this field is the study of the perfect absorption of sound by using ultra-thin absorbers. The challenge is to absorb waves in deep-subwavelength scale (where the wavelength is much larger than the length of the system). Perfect absorption is of particular interest for many applications such as energy conversion, time-reversal technology, coherent perfect absorbers or soundproofing, among others [53, 54]. Porous materials, microperforated plates and classical absorbing materials present good absorption from frequencies greater than $c / 4 L$, where $L$ is the length of the material. For lower frequencies, resonators are needed to obtain good values of absorption. Open lossy resonators present leakage of energy due to the aperture and inherent losses given by dissipative mechanisms.

The achievement of very efficient low-frequency broadband absorption is the result of a fine tuning of the structure that leads to the critical coupling of resonant modes [55]. Perfect absorption is achieved when the critical coupling 
condition is fulfilled, which can be done by carefully modifying the inherent leakage quality factor $Q_{\text {leak }}^{-1}$ to be equal to the dissipation quality factor $Q_{\text {loss }}^{-1}$ of the system $[56,57]$. When this happens, the system and the medium present impedance matching and maximal absorption depending on the configuration. The solution of this challenge requires to solve a complex problem: reducing the geometric dimensions of the structure while increasing the density of states at low frequencies and finding the good conditions to match the impedance to the background medium [58]. Previous works [55, 59] introduced the complex frequency plane of the reflection coefficient, by considering a complex frequency $\omega=\omega_{r}+i \omega_{i}$ in the study, as an efficient tool to design broadband acoustic absorbers based on resonators in the low frequency range. In that case, the tuning of the losses and of the geometric characteristics of the sub-wavelength resonators lead to the crossing of the complex zeros of the reflection coefficient with the real frequency axis which signifies the perfect absorption condition.

Romero-García et al. [53] reported, both analytically and experimentally, perfect and broadband absorption for audible sound by critical coupling, by means of a sub-wavelength multi-resonant scatterer (SMRS) made of a plateresonator/closed waveguide structure. Figure 1.13 shows the complex frequency plane in which the trajectory of the zero of the reflection coefficient can be observed, as well as the absorption coefficient for three different resonating structures and the evolution of the sound absorption coefficient, $\alpha$. As it can be seen in Figure 1.13a), the first zero of the reflection coefficient crosses the real axis, meaning that the critical coupling condition has been fulfilled and, as a consequence, perfect absorption is achieved as demonstrated in Figure 1.13b) (for the black line).

Using the concepts of slow sound and critical coupling, an ultra-thin acoustic metamaterial panel for perfect and quasi-omnidirectional absorption was theoretically and experimentally conceived by Jiménez et al. [58]. The system was based on a periodic distribution of thin closed slits whose upper wall was loaded with Helmholtz resonators. This configuration produced slow sound propagation shifting the resonance of the slit to $\lambda / 88$. As it can be observed in Figure 1.14, almost perfect sound absorption was obtained for a broad range of angles, being $\alpha>0.9$ for incident waves with $\theta<60^{\circ}$.

The solution to the problem of quasiperfect absorption in transmission by using monopolar resonators has recently been achieved by Jiménez et al. [59]. To do so, the authors used monopolar resonators to critically couple the symmetric and antysimmetric problems. The proposed solution consisted of a panel perforated with a periodic arrangement of open slits. As in the previous case, the upper wall of the slits was loaded with $N$ identical Helmholtz resonators. The 

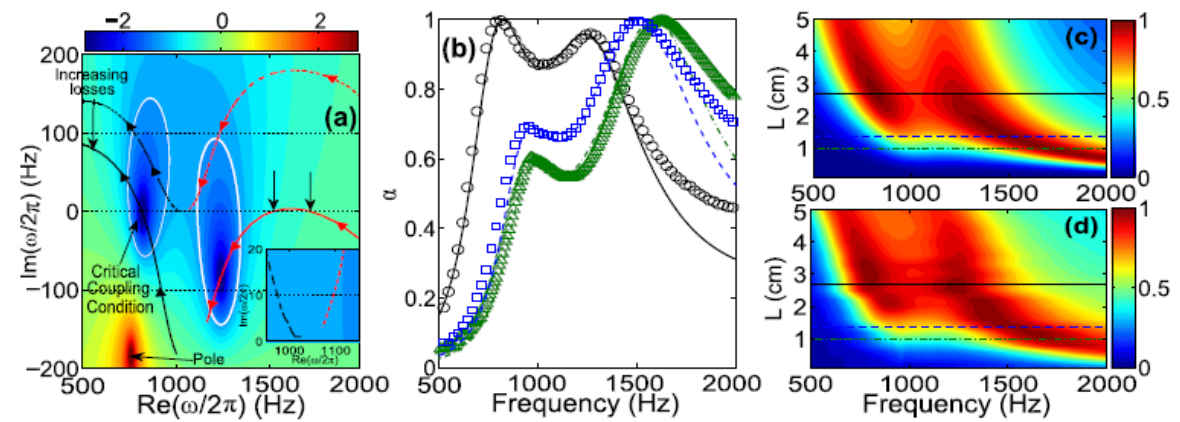

Figure 1.13: (a) Represents the complex frequency map of $\log |R|$ for the SMRS with $L=2.71 \mathrm{~cm}$. Black dashed (continuous) and red dashed (continuous) lines represent the trajectory of the zero of $|R|$ for the first and second resonances for the lossless (lossy) case as $L$ is increased (sense of the increasing shown by arrows over the lines). White continuous lines show the isoline $\alpha=0.9$. Inset shows a zoom of the complex frequency map. In (b) green dot-dashed (green open triangles), blue dashed (open blue squares) and black continuous (black open circles) lines show the analytical (experimental) absorption coefficients for the cases $L=1 \mathrm{~cm}, L=1.37 \mathrm{~cm}$ and $L=2.71 \mathrm{~cm}$ [horizontal lines in (c,d)] respectively. (c,d) show the theoretical and experimental maps for the dependence of $\alpha$ on both the length of the cavity and the frequency. Figure extracted from [53].

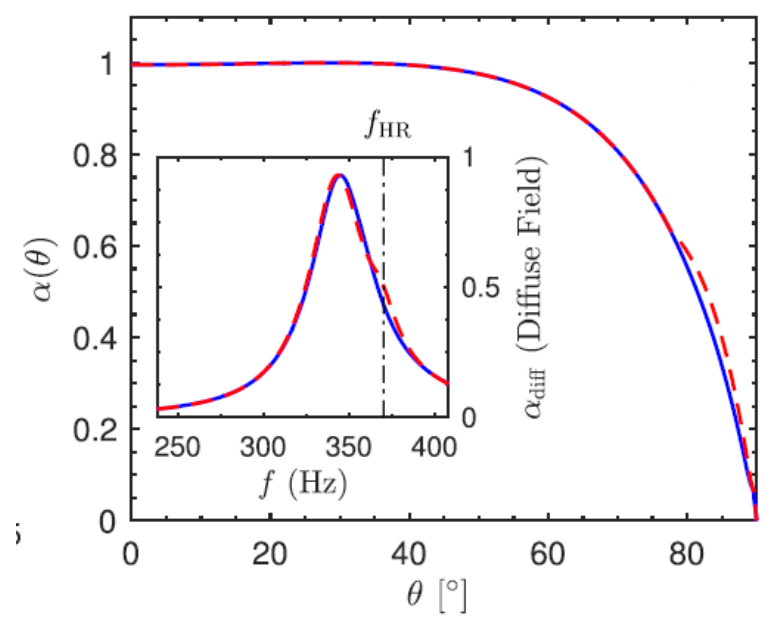

Figure 1.14: Absorption peak as a function of the angle of incidence calculated by the effective parameters (dashed red line), transfer matrix method (solid blue line). The inset shows the absorption coefficient in diffuse field as a function of the frequency. Figure extracted from [58]. 
a)

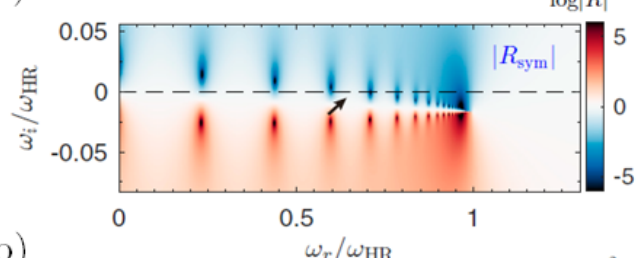

b)

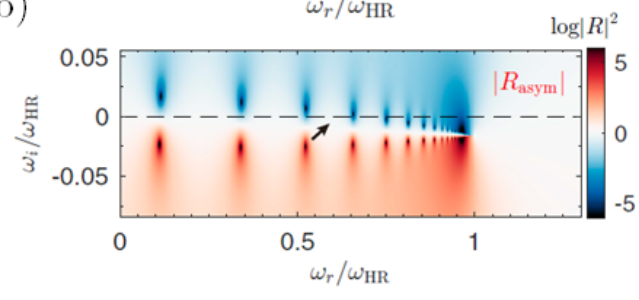

c)

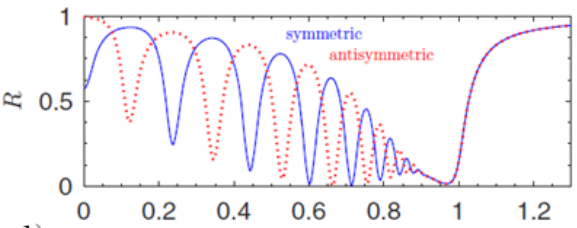

d)

$\omega / \omega_{\mathrm{HR}}$

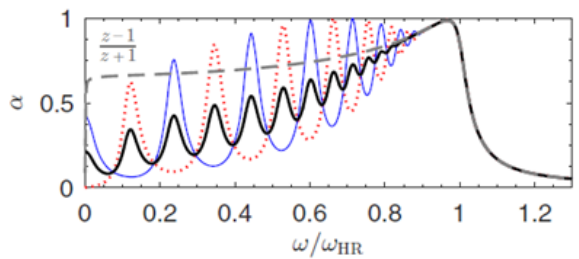

Figure 1.15: a,b) Complex frequency representation of the relation coefficient for the symmetric and antisymmetric problem, respectively. c) Reflection coefficient at the real axis for symmetric (solid blue line) and antisymmetric (dotted red line). d) Absorption for symmetric (solid blue line), antisymmetric (dashed red line), total (thick solid black line) and impedance matching condition (dashed grey line). Figure extracted from [59].

system was sufficiently large to accurately be described as a slab of material with the effective parameters. This study underlined the need of breaking the symmetry of the system in order to achieve perfect absorption in transmission or the use of degenerated resonators with symmetric (monopolar) and antisymmetric (dipolar) resonances. As it can be seen in Figure 1.15a) and 1.15b) the cavity modes (corresponding to the poles in both figures) accumulate below the resonance frequency of the resonators due to dispersion. However, it can be observed that some of the zeros of the reflection coefficient are located on the real axis. This means that the structure is critically coupled and, as Figure $1.15 \mathrm{~d})$ shows, perfect sound absorption is achieved in that problem.

One step further was given in this problem when Jiménez et al. [54] theoretically, numerically and experimentally reported perfect, broadband and asymmetric sound absorption by using subwavelenght thickness panels. In this work, the authors achieved flat and perfect absorption over a frequency range covering from 300 to $1000 \mathrm{~Hz}$ using a rainbow trapping absorber (RTA) composed of nine resonators. Three conditions must be simultaneously fulfilled to obtain broadband and perfect absorption in a problem in transmission: $(i)$ the zeros of the eigenvalues of the scattering matrix must be located on the real frequency axis, $(i i)$ the zeros of both eigenvalues, $\lambda_{1,2}$, must be at same 

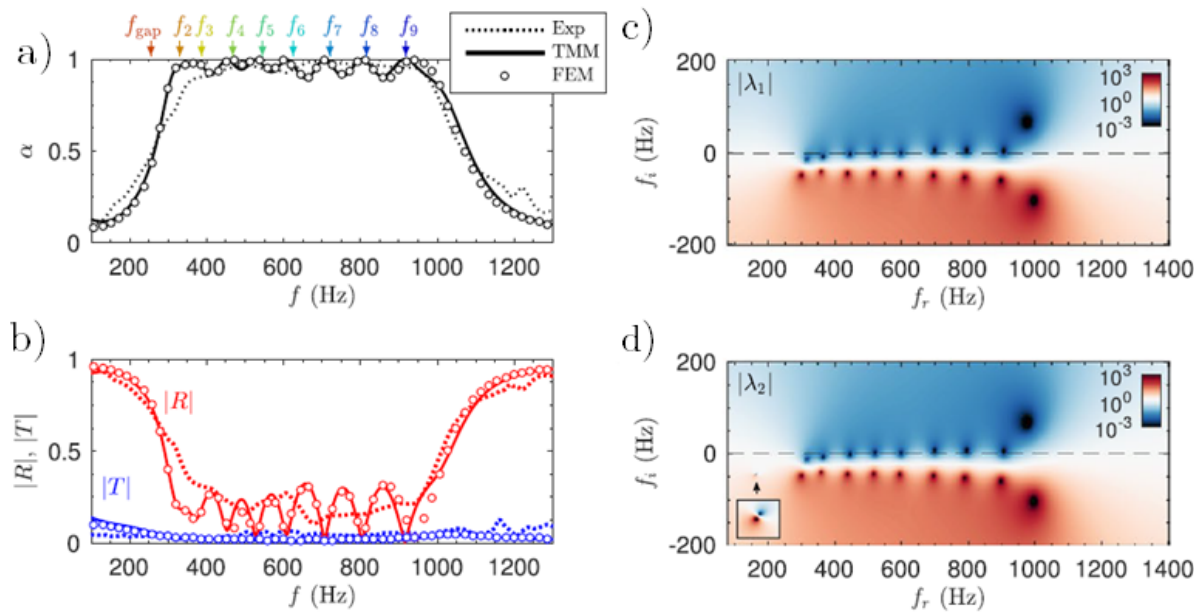

Figure 1.16: a) Absorption obtained by using the Transfer Matrix Method (solid line), Finite Element Method simulations (circles) and measured experimentally (dotted line). b) Corresponding reflection (red curves) and transmission (blue curves) coefficients in amplitude. c,d) Complex frequency representation for the eigenvalues of the scattering matrix, $\lambda_{1,2}$. Colormap in $10 \log _{10}\left|\lambda_{i}\right|^{2}$ scale. Figure extracted from [54].

frequencies, and (iii) the quality factor of the resonances must be low to overlap in frequency. The results of the study are shown in Figure 1.16. This figure shows the peaks in the absorption coefficient and its association to the resonance of each of the nine elements of the system, as well as the zero-poles diagram in which it is possible to observe that the requirements for perfect sound absorption are fulfilled.

The studies that have been mentioned make use of the critical coupling condition to achieve perfect sound absorption in different systems and configurations. It is worth nothing that considering the losses in the system is crucial for a correct modeling of the behavior of a system, and tuning them is mandatory to achieve the conditions for perfect absorption. This can be done by optimizing the geometrical parameters of the elements composing the system, as will be detailed in the following chapters. 


\subsection{Aim of the work}

Along the state of the art, the importance of the understanding of the characteristics of the acoustic environment in the vicinity of the launch pad, as well as the elements contributing to it, has been detailed. This phenomenon includes economical and security consequences. Despite this fact, there is still little knowledge on the characteristic of the sources and the behavior of the ground facilities concerning sound scattering, diffusion and absorption.

The strong consequences of the high acoustic pressure make necessary the mitigation of the load affecting the rocket. Thus, the activity is intended to study in depth the acoustic behavior of the launch pad and develop new methods for the mitigation of the sound pressure levels generated during the ignition and first stages of the lift-off of the spacecraft. Different mechanisms can be used in order to attenuate the acoustic field, such as sound diffusion, absorption or diffraction, among others. A solution based on the diffusion properties of sound waves by sonic crystals (detailed in the next Chapter) was previously studied by our research group. Keeping in mind the conclusions of this study, we decided to study the mitigation properties of the acoustic load by using sound absorption.

This Thesis is devoted to the physical understanding of a proposed system for noise reduction based on the use of Helmholtz resonators and the development of devices based on them for sound absorption. The long-term purpose would be to design structures, that should be placed on the ground facilities, more precisely on the launch pad, and whose objective is the mitigation of the noise generated by launchers in the initial phases of the flight. The general objective of this Thesis is the proposal of a system that can be used for mitigating the acoustic field that the spacecraft experiences during the first stages of the liftoff and that, at the same time, does not require substantial modifications of the existing launch pad.

This Thesis is organized as follows:

The state of the art and literature research has been presented in this Chapter. After this, and also based on the problem of the high acoustic pressure released in the first stages of the launch of spacecrafts, the results of two previous works of our group are shown in Chapter 2. The first work is based on the study of a mock-up of the VEGA launch pad. The second one studies the diffusive properties of sonic crystals (SCs), which could be used in order to mitigate the acoustic field affecting the spacecraft. Preliminary results already exist in the context of precursor studies on sound absorption by Helmholtz resonators. 
Showing the high potential of sound level mitigation of these systems due to their properties and possibility to be tuned for the desired range of frequencies.

The fundamentals of the Transfer Matrix Method (TMM) and the critical coupling condition for perfect sound absorption are presented in Chapter 3. These concepts are applied to simple resonant systems in two different configuration: a single plate in transmission and a single Helmholtz resonator showing the problem in reflection. Chapter 4 shows the experimental evidence of critical coupling condition for perfect sound absorption for a single frequency by Helmholtz resonators (HRs).

Once we have set the context of the problem to be faced and the fundamentals of the methods, we design a system composed by five HRs. The geometry of the HRs is tuned in order to achieve perfect sound absorption in a given frequency range by using the critical coupling condition. This system is studied by using an impedance tube in order to measure the sound absorption coefficient of the system.

Finally, a 2D array of HRs is studied in Chapter 6 . This system combines the effects of absorption due to the resonances observed in the previous Chapter, with the effects of diffusion. The combination of these properties increase the performance of the system for the noise mitigation at the launch pad. 



\section{Chapter 2}

\section{Preliminary results}

The state of the art and literature on high acoustic pressure level mitigation methods has been presented in Chapter 1. This information is of a crucial importance for the knowledge and understanding of the problem that we are facing in this Thesis.

Preliminary results have been also obtained in previous studies carried out by our research group. These studies are oriented, on the one hand, to the understanding of the acoustic environment of the VEGA launch pad during the first stages of a rocket launch and, on the other hand, to the study of the feasibility of the use of sonic crystals for reducing the acoustic load generated in this type of events by using the diffusion of sound. In this Chapter, two studies that have been carried out by the group, giving an important knowledge for the understanding and solution of the problem of noise mitigation at the launch pad, are presented.

\subsection{Study of the acoustic environment of the VEGA launch pad}

The distribution of the acoustic load in the close area of the launcher depends on the geometric, mechanical and acoustic characteristics of the ground facilities. The acoustic environment during the lift-off determines the internal vibration responses of the vehicle and its components. For this reason, knowing 

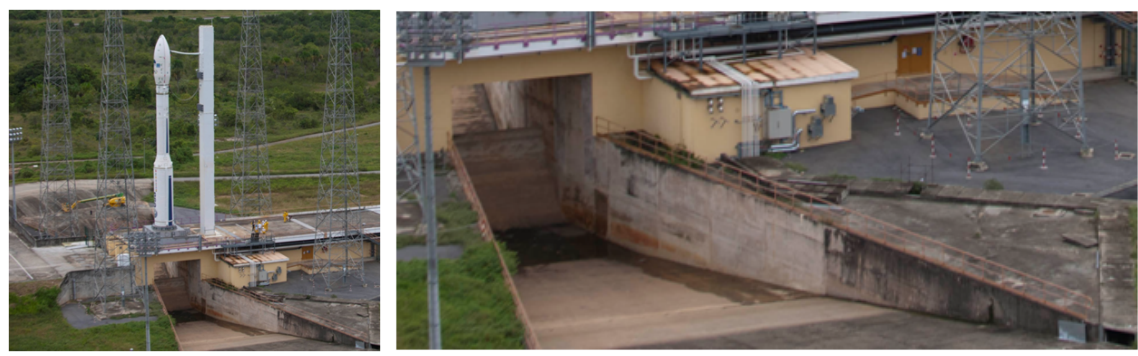

Figure 2.1: VEGA launch pad (left), and detail of the channel and the flame deflector (right).

the influence of different design parameters, as well as the spatial distribution of the acoustic load attending to geometrical aspects, is crucial. This knowledge is important to understand and to a certain extent, predict the behavior of the system composed by the launch pad and the spacecraft and propose solutions for the mitigation of the acoustic load.

In Ref. [46], the acoustic behavior of the launch pad and the evaluation of the acoustic impact of its structural elements, such as covers and flame deflectors, are studied by considering the launch pad of VEGA, a small European launch vehicle, as a reference case. In Figure 2.1 two pictures of the VEGA launch pad are shown, where two big channels are present. For this purpose, we used a subscale model with a speaker as a noise source pretending the rocket. The overall acoustic power from the plume of the advanced solid rocket is estimated to be higher than $180 \mathrm{~dB}$. A source of such characteristics could not be used in an acoustic laboratory environment. Thus, we used a speaker with the purpose of characterize the acoustic response of the launch pad.

\subsubsection{Experimental set-up}

Experimental measurements were carried out in the anechoic chamber at the Campus of Gandia of the Universitat Politècnica de València (UPV). A 1:25 (4\%) sub-scale model of the VEGA launch pad was designed and built-up with Medium Density Fiberboard (MDF). The choice of the appropriate scale was a compromise between two criteria: $(i)$ the size of the scaled launch pad to fit in the anechoic effective space of the room and $(i i)$ the working frequency band of the loudspeaker. The main acoustic elements of the launch pad are the carneaux, the gas deflectors and cover. The material can be considered acoustically rigid so that the absorption can be negligible and the acoustic impedance is orders of magnitude greater than the one of the air. The woofer 


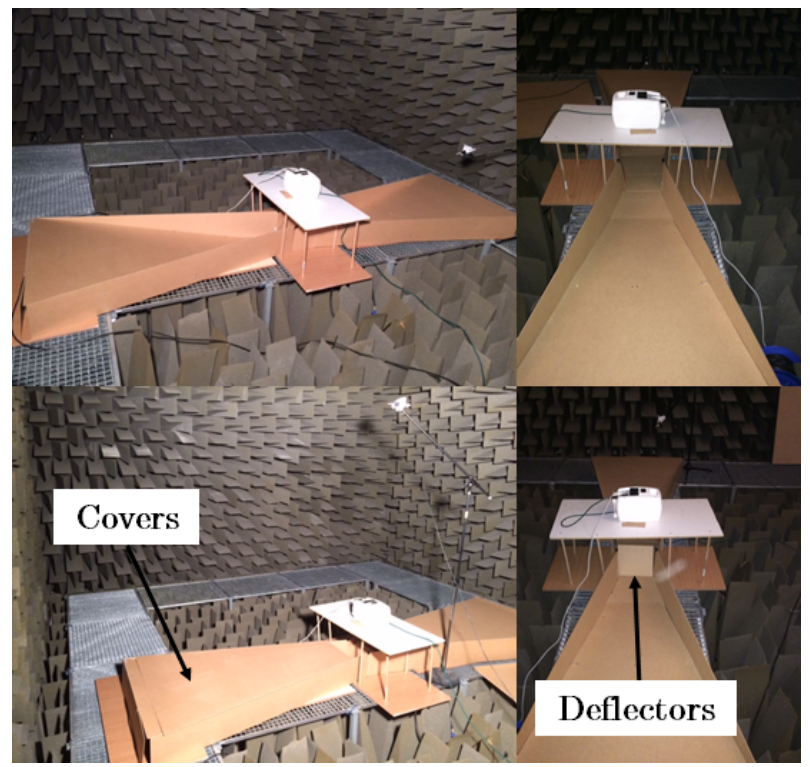

Figure 2.2: Pictures of the experimental set-up with and without covers and deflectors.

of a high quality studio monitor Genelec 8030A was used as a source. The diameter of the loudspeaker is 5 inches and it is suitable to work in frequencies between 500 and $3000 \mathrm{~Hz}$. The tweeter of the monitor was disabled during the experiments in order to ensure that the woofer is the only acoustic source during the experiment. The speaker was oriented towards the gas deflectors as it corresponds to the rocket in the ignition phase.

Several pictures of the experimental set-up are shown in Figure 2.2 in different configurations (with and without covers and deflectors). The receiver was a free-field microphone of $1 / 2$ inch with flat response and an omnidirectional directivity in the working frequency range. The acoustic measurements were made in a quarter circumference of radius $1.8 \mathrm{~m}$ centered in the source with a free-field microphone, spaced every $30^{\circ}$ in azimuth. The location of the microphone in $90^{\circ}$ corresponds to the fairing level of the launch vehicle at the end of a carneaux and $0^{\circ}$ is the vertical position above the source as shown in Figure 2.3.

The purpose of this study was to analyze the acoustic behavior of the launch pad. For this purpose, white noise was emitted by the speaker to reproduce the incoherence of the original source (the nature of the real signal is a combination of the blast wave and a continuous regime of random, ergodic and 


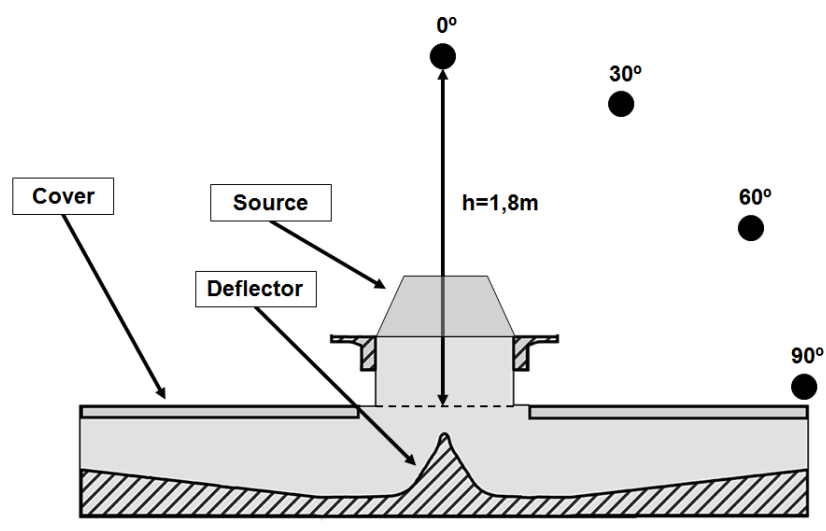

Figure 2.3: Schematic front view of the ducts with deflector and cover. Dots represent the microphone positions.

quasi-stationary noise). Acoustic measurements were time-averaged to reach stable values. The experimental set-up was designed to analyze the effect of two parameters on the acoustic behaviour of the launch pad: the presence/lack of cover and deflectors.

\subsubsection{Results}

The aim was to evaluate the acoustic effect of inserting or removing elements in the launch pad like the cover or the deflectors. In Figure 2.4 the Sound Pressure Level $(S P L)$ is presented in $\mathrm{dB}$ at $0^{\circ}$ with and without covers. Remark that the frequencies represented in Figures 2.5 and 2.6 have been transformed to the real scale $1: 1$, and correspond to the frequency range $f=[12.5,125]$ Hz. It can be seen that the effect of covers depends on frequency. In general terms, the inclusion of covers reduces the acoustic pressure above $80 \mathrm{~Hz}$. For lower frequencies a significant increase of the $S P L$ is observed. A plausible explanation of these peaks is that the resonant behaviour of the channels in the launch pad is enhanced as a consequence of closing them with covers. Variations up to $5 \mathrm{~dB}$ are observed due to the acoustic effect of covers.

In Figure 2.5 the results are presented for different microphone positions in $1 / 3$ octave bands. In this figure, the above mentioned effect of resonances on the acoustic response of covered channels at $0^{\circ}$ is reduced because of the integration in frequency bands. For other microphone positions, important variations are 


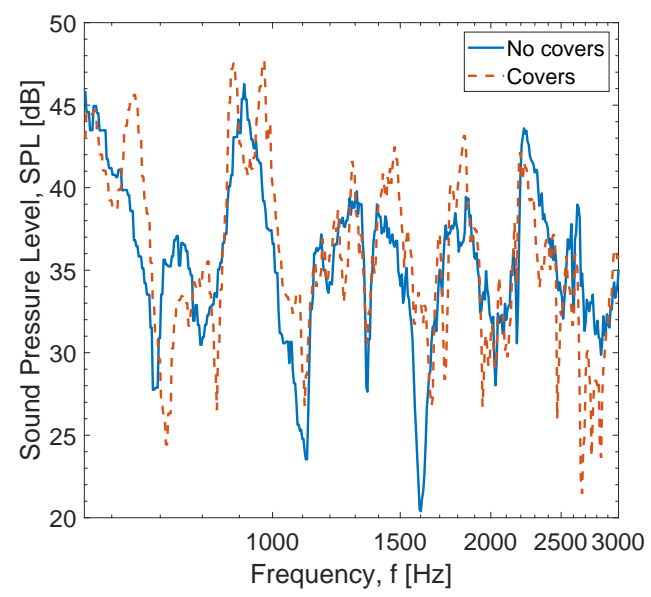

Figure 2.4: Sound Pressure Levels $(\mathrm{dB})$ with (dashed red) and without (solid blue) cover at the $0^{\circ}$ position.

also observed, but there is not a general rule. The covers mitigate the sound pressure at some frequencies, while increase the $S P L$ in some others.

The effect of removing deflectors in the acoustic behavior of the launchpad is observed in Figure 2.6, with and without covers. Although, the flame deflectors are not designed for acoustic purposes, an increase up to $4 \mathrm{~dB}$ in the $S P L$ was measured when the flame deflectors are included. It can then be concluded that they have an important acoustic effect. This result is comparable to the one presented by Madhan Kumar et al. [45], in which the authors compared deflectors with different shapes. In this study, an average increase of $3 \mathrm{~dB}$ was observed when the flame deflectors are included.

\subsubsection{Conclusions}

A $1 / 25$ scaled model of the VEGA launcher was designed, built and tested in an anechoic environment. The acoustic behaviour of the launch pad was tested. The effect of covering the channels was found to be sensitive for different angular positions. The sound pressure level reduction is important for frequencies above $80 \mathrm{~Hz}$. Cavity resonances of the semi-covered pad enhance the acoustic modal response for very low frequencies. Flame deflectors also show an important influence in the pad response. A good comprehension and 

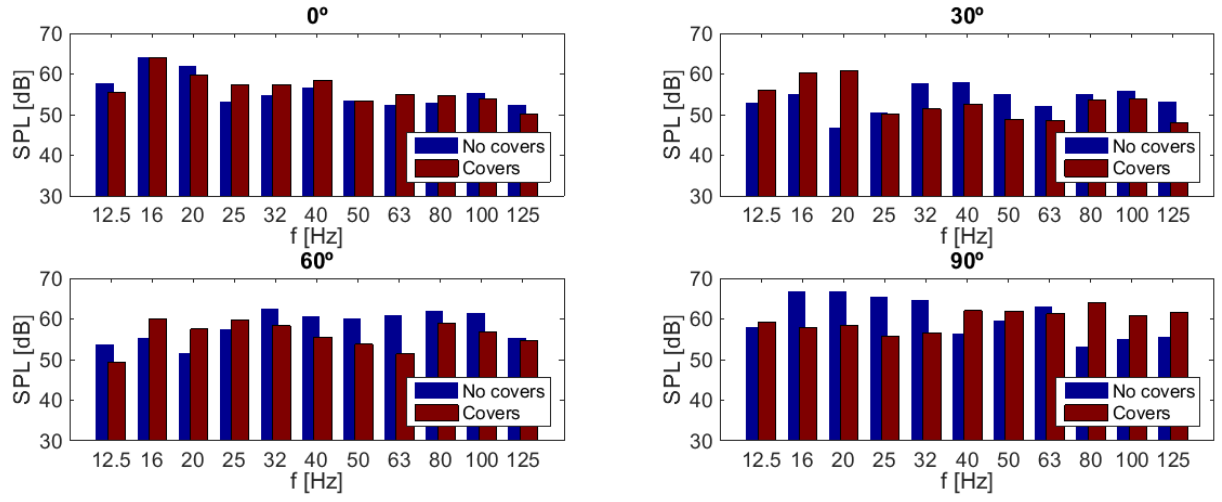

Figure 2.5: $S P L(\mathrm{~dB})$ in 1/3-octave bands with (brown) and without (blue) covers at different microphone positions.
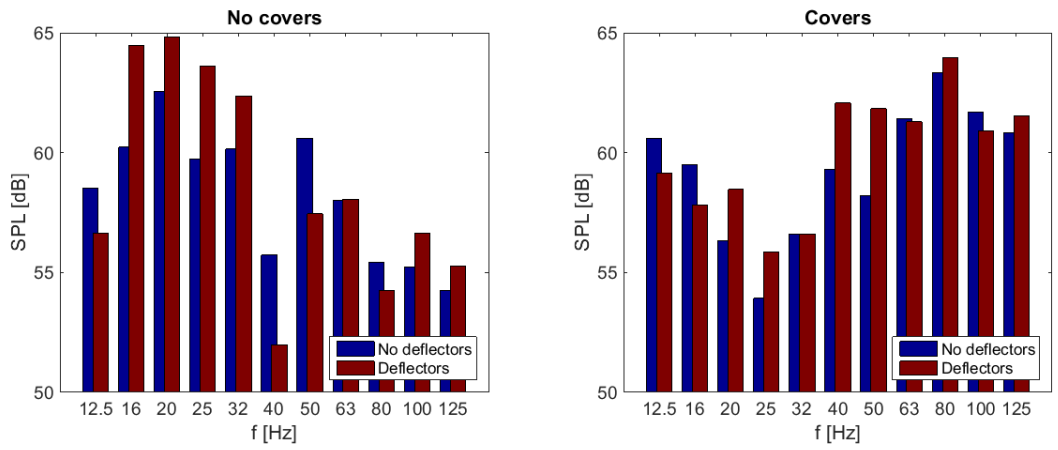

Figure 2.6: $S P L(\mathrm{~dB})$ in $1 / 3$-octave bands with (brown) and without (blue) deflectors in both configurations without (left) and with (right) covers. Measurements correspond to the $90^{\circ}$ microphone position. 

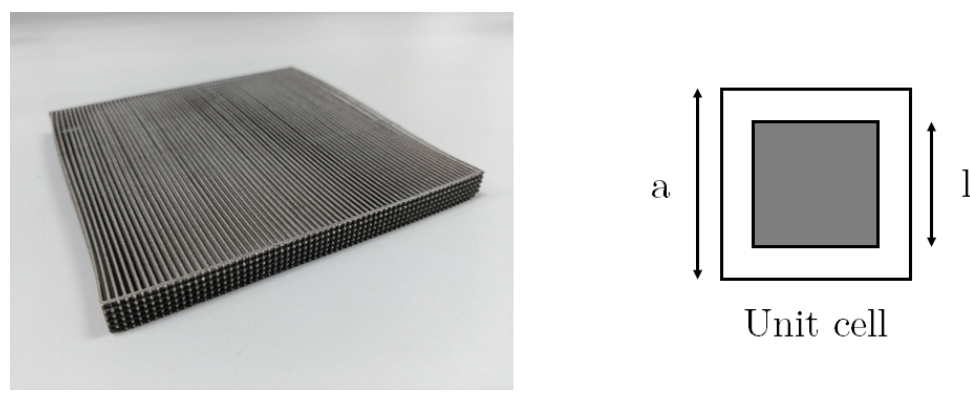

Figure 2.7: 2-D sonic crystal composed by rods of aluminum (left) and scheme of a unit cell (right).

a precise evaluation of different parameters is necessary in order to determine its influence in terms of $S P L$ in the launch pad area.

\subsection{Sonic crystals for noise reduction at the launch pad}

The feasibility of the use of Sonic Crystals (SCs) in reducing the impact of the backward reflected field emitted by a sound source in a simplified scaled model of a launch pad is shown in [48] and [49]. The main conclusions of the Innovation Triangle Initiative (ITI) project 'Sonic Crystal for Noise Reduction at the Launch Pad', developed for the European Space Agency and finished in February 2016, are summarized in this section.

\subsubsection{Introduction}

In the late 80's, the works developed by Yablonovitch [60] and John [61] laid the foundations for the study of electromagnetic band gaps in photonics through the analysis of periodic dielectric structures. A few years later, in 1993, Kushwaha et al. [62] extended these results to the field of phononics by means of the study of a repetitive structure made up of two materials with different elastic properties.

A sonic crystal (SC) consists of a periodic array of rigid elements or scatterers immersed in a host fluid medium [47] and thus only longitudinal waves are allowed to propagate. This periodicity is given by an element, known as unit cell, which is a fundamental unit that is repeated in space (see Figure 2.7) following a lattice constant, $a$. The filling fraction, $f f$, is the parameter that evaluates the ratio of the space occupied by the scatterers in the unit cell. 
These systems allow the propagation of waves at some frequencies while other ranges of frequencies, known as band gaps, are forbidden. At band gaps, the destructive interferences due to Bragg's scattering is activated and, as a consequence, low transmission through the structure is expected in agreement with the presence of band gaps due to periodicity [48]. This phenomenon has been widely studied along the last decades both theoretically and experimentally $[47,62-65]$ and has increased the interest of SC due to the several number of applications as, for example, wave filtering [66], waveguiding [67, 68] or focusing [69] among others.

They present a rich behavior as a function of the frequency. In the long wavelength regime (low frequencies), where the wavelength is much larger than the lattice constant, $\lambda>>a$, these structures behave as homogeneous materials with effective parameters (for example, effective sound speed and effective density). It corresponds to the homogenization regime. As $\lambda$ approaches the SC's period, $a$, these systems become sensitive to the periodicity, and it enters in the diffractive regime, where scattering effects dominate. In this regime, sound waves can be guided [70], focused [71], or deviated [72, 73] in particular directions. Also, the band gap appears in this regime, where transmission is extremely reduced (evanescent waves) and waves impinging the structures are reflected.

Different mechanisms have been explored that contribute to sound reduction when using SCs. At some frequencies, inside the band gap of the structure, the sound wave will be reflected back, however dispersed according some singular pattern depending on the type geometrical parameters of the SC. As a result, direct reflections coming from the launch pad are reduced in magnitude. For frequencies outside the band gap, sound penetrates in the structure and can be manipulated by different means (absorption, deviation, channeling).

The reference situation (the one that is wanted to be improved by using the diffusivity of the SC) in Ref. [48] is a sound source (the rocket) placed over a flat rigid reflector (the ground), and the sound levels around the location of the source will intended to be reduced by placing different periodic arrangements of rigid scatterers over the ground. 


\subsubsection{Design and manufacturing of the Sonic Crystals}

Four SCs were designed as test samples attending two parameters, the unit cell, that defines the lattice symmetry of the structures, and the filling fraction, that indicates the ratio of space occupied in the unit cell by the solid elements. Four different SCs have been studied considering square and triangular lattice symmetries, each with high and low filling fraction:

- Square lattice / Low filling fraction: SL

- Square lattice / High filling fraction: SH

- Triangular lattice / Low filling fraction: TL

- Triangular lattice / High filling fraction: TH

All the SCs are composed by beams (scatterers) with square cross-section, and the size of the structures is $7.7 \times 98.68 \times 97.6 \mathrm{~mm}$. The lattice constant, $a=1.45 \mathrm{~mm}$, is the same in all the SCs (see Figure 2.8). Each SC is made of an arrangement of $n \times m$ square rod scatterers of side length $l$, placed in a square or triangular array (depending on the case) which lattice constant is $a$. Therefore, the filling fraction is defined as $f f=l^{2} / a^{2}$ for those SCs having a square lattice and $f f=4 l^{2} /\left(\sqrt{3} a^{2}\right)$ for those having a triangular one. The filling fractions for each SC are: $9.63 \%$ for the SL, $38.53 \%$ for the SH, 22.24 $\%$ for the TL and $70.30 \%$ for the TH.

The band gap associated to the $\Gamma \mathrm{X}$ direction, i.e., normal incidence to the SC, at Bragg's frequency, is around $f_{B}=c_{0} / 2 a$, being $c_{0}$ the speed of sound. Thus, the band gap will appear at $f_{B}=517 \mathrm{kHz}$.

The structures have been manufactured by Selective Laser Melting (SLM). This is an Additive Manufacturing (AM) process in which a three-dimensional part is built layer by layer by using a laser scanning over a previously deposited powder bed. The SCs have been built in the titanium alloy Ti-6Al-4V (also called Ti64), known in this way because it contains an average of $6 \%$ of aluminum and $4 \%$ of vanadium. A picture of the manufactured SCs can be observed in Figure 2.9. 


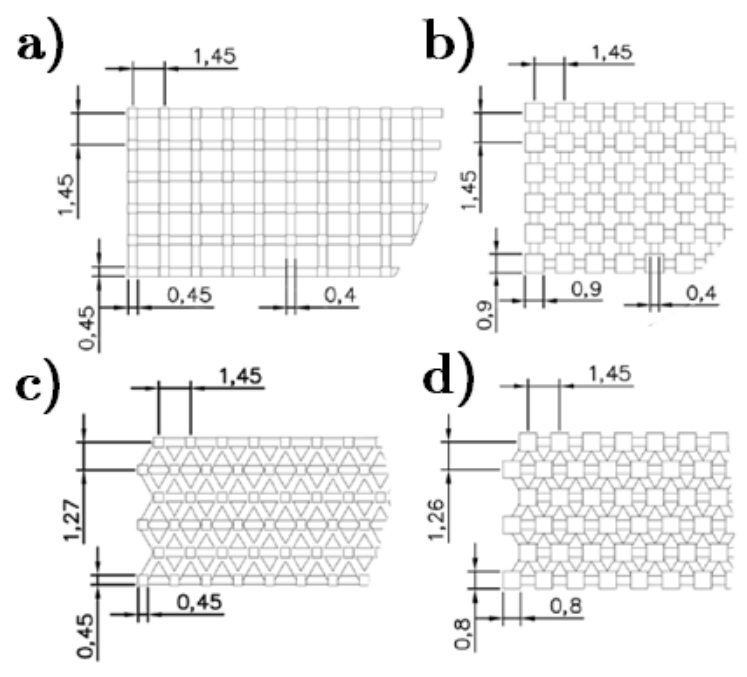

Figure 2.8: Details of the lattice geometry of the four 2D SCs. a) SL, b) SH, c) TL, d) TH.

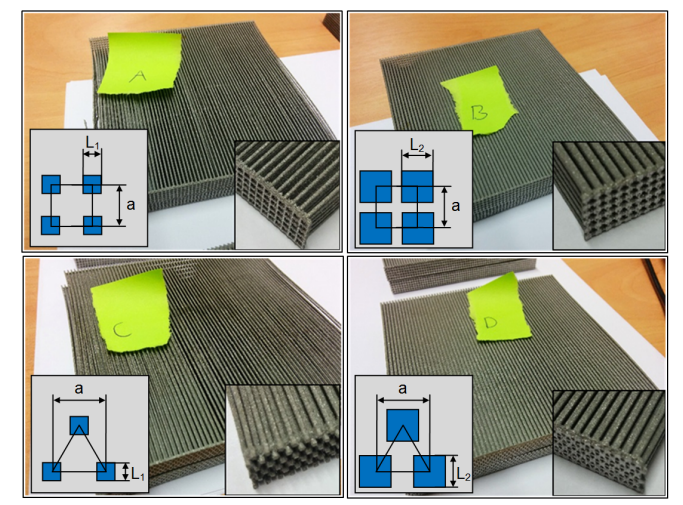

Figure 2.9: 2D SCs manufactured in Ti-6Al-4V. 


\subsubsection{Definition of the parameters to study}

- Diffusion coefficient

In the problem that we are considering in this section, the sound impinging a surface can be either absorbed or reflected (transmission is not present in this problem because the $\mathrm{SC}$ is placed in front of a rigid backing). These phenomena can be characterized by means of the sound absorption coefficient, $\alpha$, and the sound reflection coefficient, $R$, respectively. At the same time, the sound energy that is reflected back can be reflected in a specular direction, by following Snell's law, or in a diffuse way, spread in all directions according to a given pattern. It is worth nothing here that viscothermal losses are low in the range of frequencies of interest, so absorption can be neglected in this study $(\alpha \simeq 0)$.

The diffusion coefficient, $d$, is derived from measurements over a semicircumference surrounding the test specimen along $n$ angular positions and by using the following equation [74]:

$$
d=\frac{\left(\sum_{i=1}^{n} 10^{S I L_{i} / 10}\right)^{2}-\sum_{i=1}^{n}\left(10^{S I L_{i} / 10}\right)^{2}}{(n-1) \sum_{i=1}^{n}\left(10^{S I L_{i} / 10}\right)^{2}},
$$

where $S I L_{i}$ is the Sound Intensity Level of the reflected sound in the $i$-th angular position, defined as

$$
S I L=10 \log _{10} \frac{I_{i}}{I_{0}}
$$

For the sake of simplicity, we also normalize the diffusion coefficient with respect to the rigid backing of the structure,

$$
d_{n}=\frac{d-d_{r e f}}{1-d_{r e f}}
$$

where $d$ and $d_{r e f}$ are the diffusion coefficients for the whole structure and for the rigid backing, respectively. $d_{n}$ gives the diffusion coefficient of the structure compared to the one of the flat rigid backing, meaning that, for $d_{n}=0$ the structure has the same behavior as the reference case. 


\section{- Insertion Loss in Reflection}

The Insertion Loss $(I L)$ is defined as the difference in sound pressure levels measured before and after a barrier or silencer installed in the line between the source and the receiver [75]. Here we propose a set of new definitions based on the general idea of characterizing the insertion of a test sample adapted to the real problem of the dispersion of the acoustic wave reflected by the launch pad. For this reason, since both the source and the receiver are placed at the same side, we propose an extension of the concept of $I L$ in order to obtain this magnitude for systems in reflection (Insertion Loss in Reflection, ILR).

The sound pressure, $p$, as a function of the position, $x$, and the frequency, $f$, i.e. $p(x, f)$, is obtained in a certain number of measuring points placed in front of the system to be studied. Since the $I L$ is a relative parameter, based on the comparison of the system under study and a reference case, it is necessary to identify which one is going to be this reference. In our study, we use a rigid flat wall.

By considering this definition, one can obtain the $I L R$, in $\mathrm{dB}$, as follows:

$$
I L R=10 \log _{10} \frac{\left|p_{r e f}\right|^{2}}{\left|p_{S C}\right|^{2}}=L_{r e f}-L_{S C},
$$

where, $p_{\text {ref }}$ and $p_{S C}$ are the pressures measured in the case of reference and placing the system of SCs, respectively, and $L_{r e f}$ and $L_{S C}$ the sound pressure level in both cases.

\subsubsection{Experimental set-up}

A simplified mock-up of the VEGA launch pad, placed at the ESA's spaceport in French Guiana, made of a metallic plate (iron) was manufactured. The experimental measurements have been carried out in a water tank at frequencies corresponding to the ultrasonic regime. The acoustic source used is an ultrasonic transducer Olympus Panametrics A301S, with a diameter of 25.4 $\mathrm{mm}$ and a resonance frequency of $500 \mathrm{kHz}$. The receiver is a miniature probe hydrophone Reson TC4038, able to work in a range from $10 \mathrm{kHz}$ to $800 \mathrm{kHz}$. The hydrophone is mounted on a robotized axis system in order to allow the mapping of the surface in front of the system.

The range of frequencies for this study is from $200 \mathrm{kHz}$ to $800 \mathrm{kHz}$. In this range, both the sound source and the receiver have linear responses. The 

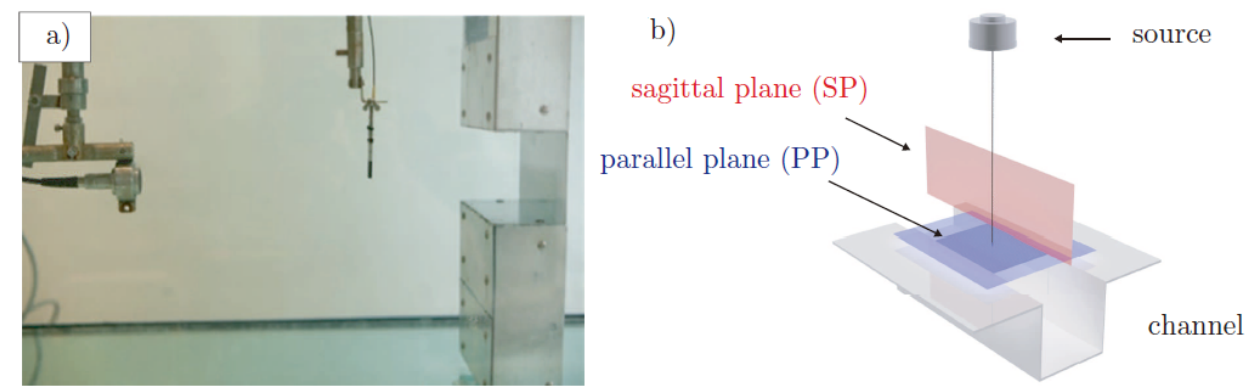

Figure 2.10: a) Experimental set-up composed by the sound source (ultrasonic transducer), the receiver (hydrophone) and the reference backing mimicking the launch pad. b) Measured planes in the experimental work.

experimental set-up can be observed in Figure 2.10a). Two planes have been measured: sagittal $(160 \times 80 \mathrm{~mm})$ and parallel $(140 \times 140 \mathrm{~mm})$ to the system (see Figure 2.10b)). Sagittal planes are measured to represent accurately the radiation pattern of the incident and reflected fields from the backings and the SCs, while the parallel planes are used to compute the far-field by means of spatial Fourier transforms. Both planes have been chosen to fulfill two conditions: i) near-field data can be measured, and ii) the incident and reflected waves can be separated in all the measurement points.

\subsubsection{Results}

In order to compare the results obtained with the four SCs, we compare their reflection properties with those of a flat rigid reflector with the same dimensions placed over a flat rigid backing.

The scattering waves in the far-field provide information about the direction of the acoustic waves reflected by the structure [49].

\section{- Diffusion coefficient}

If the incident field is reflected only in one specific direction, the diffusion coefficient is zero $(d=0)$. As opposite, if the incident wave is reflected with the same intensity in all directions (it is equally spread in space), the diffusion coefficient is equal to one $(d=1)$.

The experimental diffusion coefficient in the range of frequencies under study $(200$ to $800 \mathrm{kHz}$ ) of the reference case (a flat rigid reflector placed over a flat 


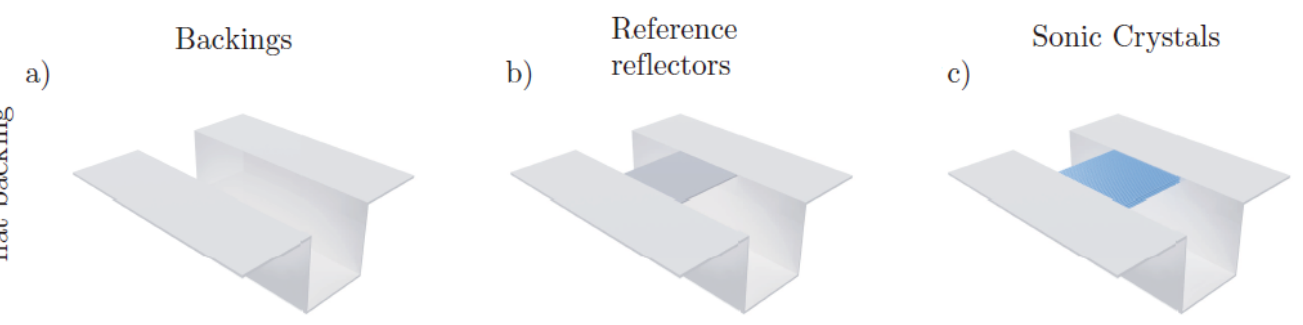

Figure 2.11: Scheme of the measured configurations: a) flat rigid backing, b) flat rigid reflector and c) SC on the flat rigid backing.

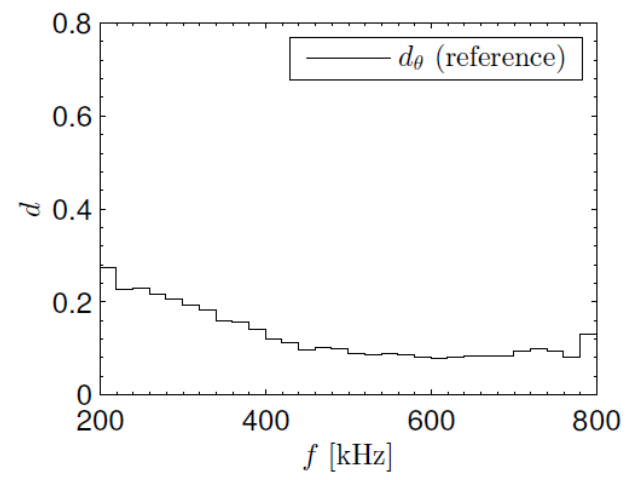

Figure 2.12: Diffusion coefficient of the reference flat panel.

rigid backing) is shown in Figure 2.12. The panel does not behave as a perfect reflector due to its finite size, which causes diffraction due to border effects.

In the same way, the experimental diffusion coefficient of the four SCs is depicted in Figure 2.13a). In order to have a better observation of the results, the normalized diffusion coefficient is plotted in Figure 2.13b), considering as a reference the diffusion coefficient of a flat panel. At low frequencies, the SCs behave in a similar way to the rigid panel. However, for frequencies above the band gap the SCs present a more diffusive behavior, achieving values up to 3 or 4 times greater than the ones corresponding to the flat panel. In this figures, it can be observed that the SC presenting triangular lattice and low filling fraction (TL) has the highest diffusion coefficient, while those having a high filling fraction ( $\mathrm{SH}$ and $\mathrm{TH}$ ) have a more similar behavior to the reference case. 

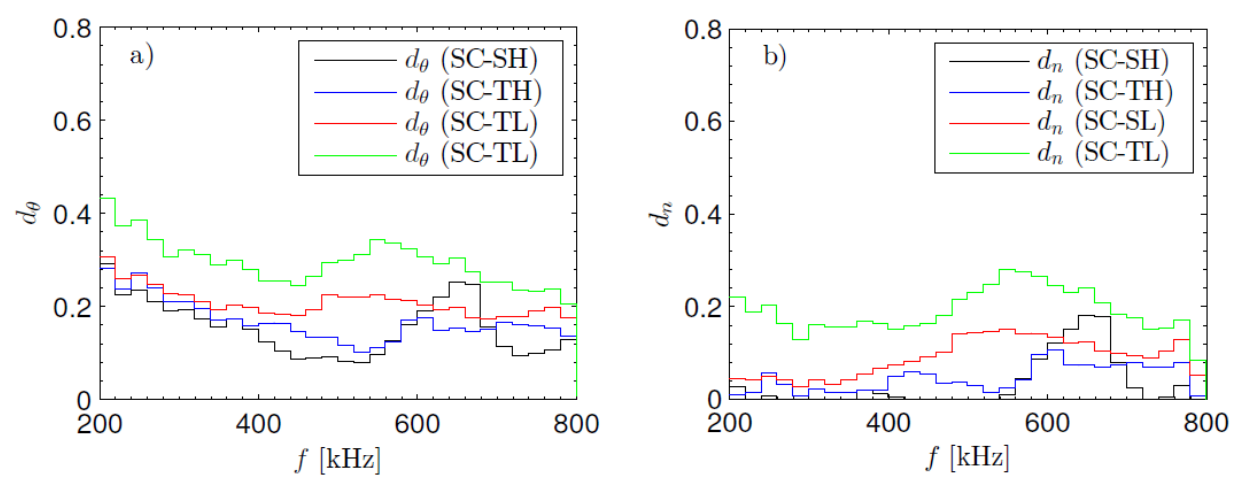

Figure 2.13: a) Diffusion coefficient of the four SCs. b) Normalized diffusion coefficient of the four SCs, taking as a reference the diffusion coefficient of a flat panel.

- Insertion Loss in Reflection

The Insertion Loss in Reflection $(I L R)$ is used to compare the reflected field by using a SC sample and the one measured in a reference configuration (in this case, a flat rigid panel). The resulting $I L R$ for each SC is shown in Figure 2.14 , in which the obtained $I L R$ is integrated in frequency. The transducer is located at the top of the map $(z=0 \mathrm{~mm})$, while the SC is located at the bottom $(z=230 \mathrm{~mm})$. The color scale is in logarithmic scale, meaning that $I L R>0$ (reddish regions) corresponds to a lower total field by using the SC sample and, thus, an $I L R<0$ (blue regions) means an increase of the total field.

As it can be observed in Figure 2.14, the $I L R$ is positive in the central part (from $-20 \mathrm{~mm}$ to $20 \mathrm{~mm}$ ), in which the rocket would be placed, of all the SCs studied. These results demonstrate that the SC spreads the acoustic energy considerably if compared to the flat rigid reflector in this region and, for instance, reduces the acoustic field. Blue regions, in which the acoustic field increases, can be also observed. Nevertheless, these regions are out of the region of interest. This confirms the theory that the SCs reduce the acoustic field in the region of interest by spreading the energy in other directions different than normal. A higher $I L R$ can be observed in the case in which SCs with lower filling fraction are used.

If the result of the ILR is integrated in the region of interest and presented in frequency bands as well as its global value (red bar) (Figure 2.15), it is possible 

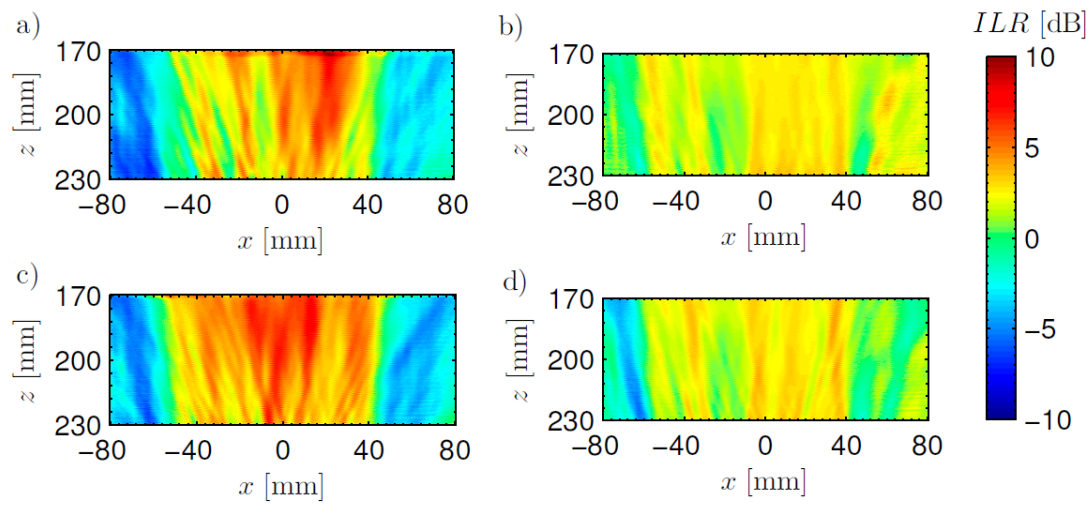

Figure 2.14: Spatial distribution of the Insertion Loss in Reflection $(I L R)$ : a) SL, b) SH, c) TL, d) TH.

to observe that there is a positive $I L R$ in all the bands. It is important to note that at the band gap frequency $(500 \mathrm{kHz})$ the $I L R$ dramatically decreases.

As it happened in the case of the diffusion coefficient, those SCs having a high filling fraction ( $\mathrm{SH}$ and $\mathrm{TH}$ ) have a more similar behavior to the reference case.

\subsubsection{Conclusions}

In Ref. [48], the potential use of sonic crystals (SCs) as noise reducing structures has been studied. A total of four different SCs have been designed attending to two main parameters: the unit cell and the filling fraction. Two different lattice symmetries (square and triangular) have been considered, each one with a high and low filling fraction, which combine into the mentioned four cases.

The diffusion coefficient reaches values of 0.25 and the $I L R$ have been experimentally measured. For SCs placed in front of the sound source in a channel with a flat bottom (with constant depth), the measured diffusion coefficient reaches values of 0.25 , and the values of $I L R$ are close to $5 \mathrm{~dB}$. The reduction of the OASPL, calculated in two octaves (restricted by the bandwidth of the source) is of $2 \mathrm{~dB}$. This represents a decrease of the $37 \%$ of the acoustic energy in front of the structures.

The results show that SCs can reduce broadband noise levels at the location of an acoustic source due to the reduction of the specular reflection and the 

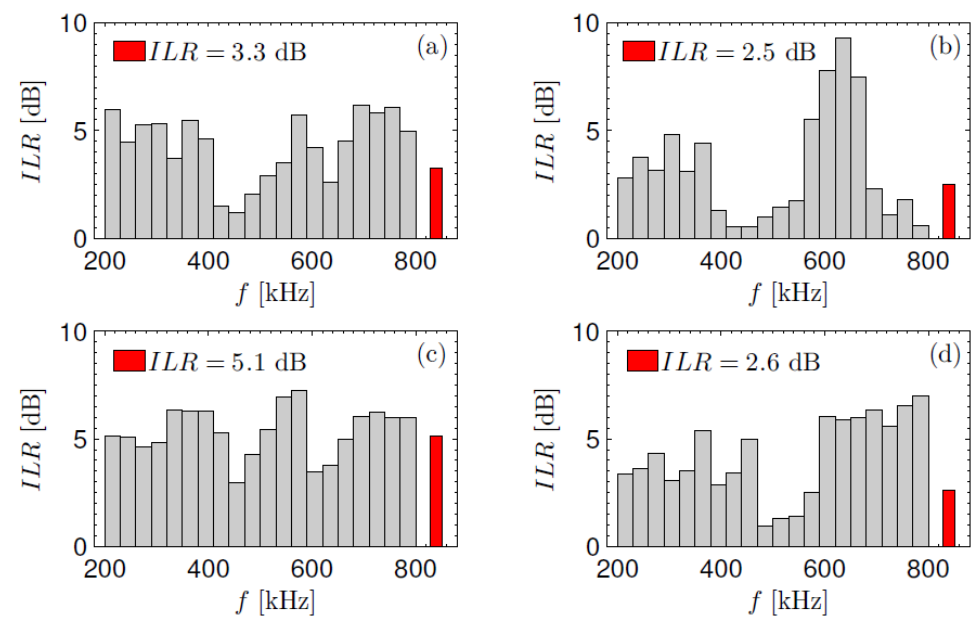

Figure 2.15: (ILR) integrated on an area of $60 \times 30 \mathrm{~mm}^{2}:$ a) SL, b) SH, c) TL, d) TH.

deflection of acoustic waves in other directions.

The works presented here described the acoustic environment in the launch pad and showed the effect of the acoustic diffusion for noise mitigation. In the upcoming chapters, we substitute the rigid diffusers of the discrete structure by a local resonator to add new physical phenomena for the mitigation of sound. 

Chapter 3

\section{Transfer Matrix Method and critical coupling condition for acoustic waves}

The preliminary studies for this Thesis, detailed in Chapter 2, showed the acoustic field in the launch pad and the diffusive properties of SCs. A good performance regarding to the diffusion of sound waves was reported. In this chapter we substitute the rigid diffusers composing the SCs by local resonators with the goal of adding new physical phenomena for the mitigation of sound.

The fundamentals of the Transfer Matrix Method (TMM) and the critical coupling condition in local resonators are introduced in this chapter. These tools can be used in order to tune the geometry of these local resonators and design perfect absorbers.

First, the TMM and the scattering problem are presented for a case in transmission and for a case in reflection. In this part, the analytical equations for finding the solution for the transmission, reflection and absorption coefficients, as well as the eigenvalues and eigenvectors of the system are detailed.

The critical coupling condition is later studied. This condition allows perfect sound absorption when there is a balance between the leakage of energy and the inherent losses of the system. The critical coupling condition is applied 
then to a problem in transmission for an aluminum square plate, and to a problem in reflection for a system composed by a HR.

\subsection{The Transfer Matrix Method}

The Transfer Matrix Method (TMM) can be used for the analysis of the wave propagation and scattering problems of acoustic, elastic and electromagnetic waves in one dimension. This method is particularly useful when the whole system can be represented into a sequence of subsystems that interact only with adjacent systems [76]. Although the TMM is a 1D model, it can be used to model $2 \mathrm{D}$ and $3 \mathrm{D}$ systems in which the sound propagation can be described in one dimension as, for example, the rainbow trapping absorber [54].

The matrix used to describe the free wave propagation in a homogeneous and isotropic material can easily be used to identify the materials' characteristic impedance and wave number, from which other acoustical quantities of interest can be calculated [77].

When the incident wave interacts with an obstacle, part of its energy is reflected back and part of it is transmitted. In case that the losses in the system are considered, some part is also absorbed. Therefore the scattering characterization of the system can be represented by the reflection, $R$, transmission, $T$, and absorption, $\alpha$, coefficients. In that case, a scattering matrix can be defined that will be related to the transfer matrix, as it will be detailed in the upcoming sections. If the complex pressure amplitude of the incident wave is $p_{i}$, that of the reflected wave $p_{r}$, and that of the transmitted wave $p_{t}$, then we can define the pressure transmission and reflection coefficients as [32]:

$$
\begin{aligned}
& T=p_{t} / p_{i} \\
& R=p_{r} / p_{i}
\end{aligned}
$$

It is important to keep in mind the limitation that TMM assumes that only plane waves propagate in one dimension and isotropic materials. 


\subsubsection{Problem in transmission: general equations for symmetric scattering}

The transfer matrix of a system, $\mathbf{M}$, extending from $x=0$ to thickness $x=L$ is used to relate the sound pressure, $p$, and normal acoustic particle velocity, $v$, between its two faces as follows

$$
\left[\begin{array}{l}
p \\
v
\end{array}\right]_{x=0}=\mathbf{M}\left[\begin{array}{l}
p \\
v
\end{array}\right]_{x=L}=\left[\begin{array}{ll}
M_{11} & M_{12} \\
M_{21} & M_{22}
\end{array}\right]\left[\begin{array}{l}
p \\
v
\end{array}\right]_{x=L} .
$$

We start considering a symmetric and reciprocal system placed into a waveguide (see Figure 3.1). An incident wave, emitted by a sound source placed at the entrance of the tube, propagates into the waveguide and interacts with the system.

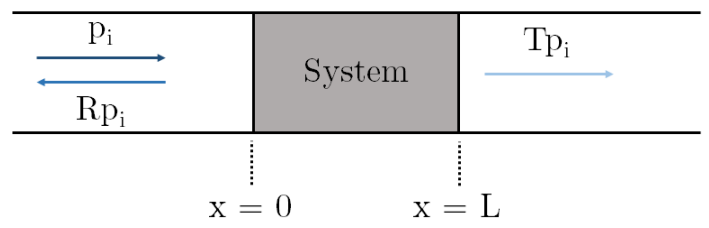

Figure 3.1: 1-D scheme of the system in transmission.

Due to the geometry of the system the scattering is symmetrical, meaning that the reflection coefficient is identical at both sides of the obstacle when the wave is emitted from left to right and reversal. This condition implies that the elements of the transfer matrix must fulfill the equation $M_{11}=M_{22}$. Moreover, since linear regime is considered, the scattering process should be reciprocal and, thus, transmission coefficients must be also identical at both sides of the obstacle. This implies that the determinant of the transfer matrix is equal to unity, $M_{11} M_{22}-M_{12} M_{21}=1$ [78].

From Eq. (3.3), we can obtain the following equations:

$$
\begin{aligned}
& p(0)=M_{11} p(L)+M_{12} v(L), \\
& v(0)=M_{21} p(L)+M_{22} v(L) .
\end{aligned}
$$


Under these conditions for a wave of unitary amplitude traveling from left to right, the pressure in the left-hand side of the system will be the result of the incident pressure plus the reflected one, that is:

$$
p(0)=1+R,
$$

and for the velocity, we deduct the part corresponding to the reflected wave that travels in the opposite direction that the incident wave and divide by the impedance of the air in the tube, that is:

$$
v(0)=\frac{1-R}{Z_{0}} .
$$

In the same way, the wave in the right part will be the transmitted one (taking into account the phase $e^{i k L}$ corresponding to the plane wave traveling to the right part, evaluated at $x=L$ ):

$$
p(L)=T e^{i k L},
$$

For the velocity:

$$
v(L)=\frac{T e^{i k L}}{Z_{0}}
$$

where $Z_{0}=\rho_{0} c_{0}$ is the acoustic impedance of air and $k$ is the wavenumber.

Combining Eq. (3.4) with Eqs. (3.5)-(3.8), we obtain

$$
\begin{array}{r}
1+R=T e^{i k L}\left(M_{11} \frac{M_{12}}{Z_{0}}\right), \\
1-R=T e^{i k L}\left(Z_{0} M_{21}+M_{22}\right) .
\end{array}
$$

By adding both equations in Eq. (3.9), we can obtain the equation for the transmission coefficient:

$$
T=\frac{2 e^{-i k L}}{M_{11}+M_{12} / Z_{0}+Z_{0} M_{21}+M_{22}} .
$$




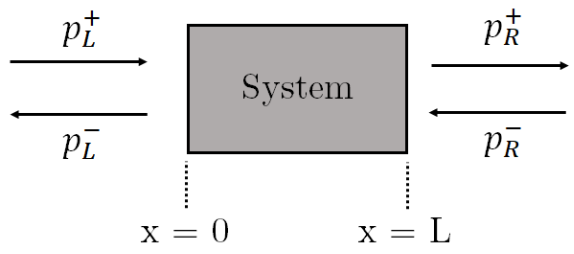

Figure 3.2: Forward and backward waves in the scattering process for the problem in transmission.

Likewise, it is possible to obtain the equation for the reflection coefficient by dividing the same equations:

$$
R=\frac{M_{11}+M_{12} / Z_{0}-Z_{0} M_{21}-M_{22}}{M_{11}+M_{12} / Z_{0}+Z_{0} M_{21}+M_{22}}
$$

In the lossless case, no absorption is produced and $|T|+|R|=1$. In the lossy case this relation is not accomplished since the absorption coefficient must be taken into account as $\alpha=1-|T|^{2}-|R|^{2}$.

\subsubsection{Problem in transmission: the scattering matrix}

The waves in the left and the right side of the obstacle can be defined in terms of plane waves as follows:

$$
\begin{gathered}
p_{L}^{+}(x)=A e^{-i k x}, \quad p_{R}^{+}(x)=C e^{-i k x}, \\
p_{L}^{-}(x)=B e^{i k x}, \quad p_{R}^{-}(x)=D e^{i k x}
\end{gathered}
$$

being $A, B, C$ and $D$ the amplitudes of the corresponding plane waves, $k$ the wavenumber and $x$ the position. The subscripts $L$ and $R$ indicate the position of the wave (left and right, respectively) with respect to the system. The superscripts + and - indicate the direction of propagation of the acoustic wave (from left to right and vice-versa, respectively). Note that the convention used is $e^{i \omega t}$.

The components of the acoustic pressure at both sides of the structure are shown in Figure 3.2 and can be defined as: 


$$
\begin{gathered}
p_{L}(x)=A e^{-i k x}+B e^{i k x}, \quad \text { for } \quad x<0 \\
p_{R}(x)=C e^{-i k x}+D e^{i k x}, \text { for } \quad x>L
\end{gathered}
$$

The linear relationship between the amplitudes of the incoming and the outgoing waves can be written in a general matrix form by means of the scattering matrix, $\mathbf{S}$ as follows:

$$
\left[\begin{array}{l}
A \\
D
\end{array}\right]=\mathbf{S}\left[\begin{array}{l}
C \\
B
\end{array}\right]=\left[\begin{array}{ll}
T & R \\
R & T
\end{array}\right]\left[\begin{array}{l}
C \\
B
\end{array}\right]
$$

where $T$ and $R$ are the transmission and reflection coefficients, respectively.

The eigenvalues of the matrix are the solutions of the equation

$$
(S-\lambda I) \vec{x}=0,
$$

where $\lambda$ is the eigenvalue to be determined and $I$ is the identity matrix. A zero eigenvalue of the scattering matrix corresponds to the case in which the incident waves can be completely absorbed [79], since they are identified as resonances of the system.

To find the eigenvalues, we consider that the determinant is equal to zero $(|S-\lambda I|=0)$. The two eigenvalues $\lambda_{1,2}$ of the system are

$$
\lambda_{1,2}=T \mp R
$$

The eigenvalues of the scattering matrix are strongly dependent of the impedance of the obstacle and, as a consequence of this, they depend on the frequency.

In the case in which one of the two eigenvalues is equal to zero $\lambda=0$, it implies that

$$
\left[\begin{array}{l}
C \\
B
\end{array}\right]=\left[\begin{array}{l}
0 \\
0
\end{array}\right]
$$




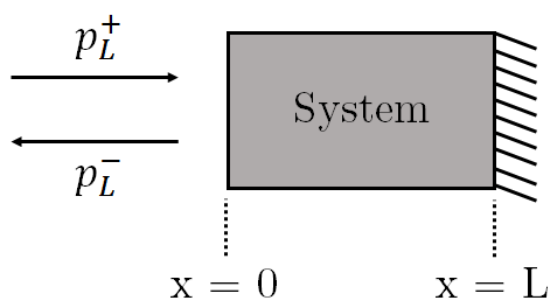

Figure 3.3: 1-D scheme of the system in reflection.

and, for instance, the acoustic energy is localized in the open system due to the fact that the amplitudes of the outgoing waves are equal to zero.

\subsubsection{Problem in reflection: general equations}

In the case that a rigid backing is considered behind the system (see Figure 3.3), no transmission occurs. In this situation, known as the problem in reflection, we consider a rigid wall placed behind the obstacle, meaning that the boundary condition is $v(L)=0$. Thus, Eq. (3.3) can be written as

$$
\left[\begin{array}{l}
p \\
v
\end{array}\right]_{x=0}=\left[\begin{array}{ll}
M_{11} & M_{12} \\
M_{21} & M_{22}
\end{array}\right]\left[\begin{array}{l}
p \\
0
\end{array}\right]_{x=L}=\left[\begin{array}{c}
M_{11} p(L) \\
M_{21} p(L)
\end{array}\right]
$$

Considering the sound pressure $p(0)$ and particle velocity $v(0)$ as in Eqs. (3.5) and (3.6), respectively, we arrive to these two equations:

$$
\begin{aligned}
1+R & =M_{11} p(L), \\
\frac{1-R}{Z_{0}} & =M_{21} p(L) .
\end{aligned}
$$

As in the previous case, dividing the two equations in Eq. (3.19), we get the equation for the reflection coefficient:

$$
R=\frac{M_{11}-Z_{0} M_{21}}{M_{11}+Z_{0} M_{21}}
$$

If no losses are considered, all the energy reaching the system is reflected back, since no transmission is allowed. Thus, $|R|=1$. Analogously to the problem in 


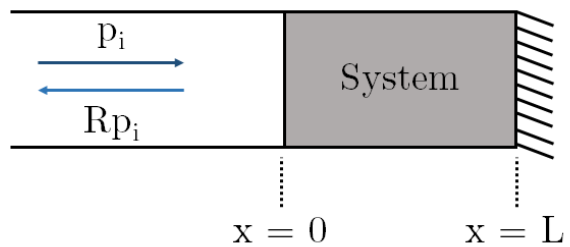

Figure 3.4: Forward and backward waves in the scattering process for the problem in reflection.

transmission, in the lossy case the sound absorption coefficient must be taken into account as $\alpha=1-|R|^{2}$. This statement will be demonstrated in Section 3.3.2.

\subsection{Critical coupling condition}

The properties of an open resonant system for sound transmission and reflection can be characterized by means of its leakage rate of energy. This leakage is produced due to the radiation of the system to the host medium and its inherent losses, such as the viscothermal or viscoelastic losses. Viscothermal losses limit the strong dissipative effects in sonic crystals [80] and in acoustic metamaterials [81]. The critical coupling condition holds when these two physical phenomena are balanced [53] and, as a consequence, perfect sound absorption can be achieved in the system. For those frequencies at which the critical coupling condition is achieved, the value of $\alpha$ reaches unity.

Reflection and transmission can be inhibited in the case of transmission systems by means of the use of degenerated critically coupled resonators with symmetric and anti-symmetric resonances. Symmetric systems present even and odd combinations of equal-amplitude waves at two input points, and the modes of the resonating element are also even and odd (corresponding to symmetric and anti-symmetric resonances). This leads to a trapping of the energy in the resonant element and, as a consequence, producing perfect absorption [58].

In the case in reflection, in which no transmission is allowed $(T=0)$, the Smatrix collapses to the reflection coefficient. The reflection coefficient itself is the eigenvalue of the scattering process. 


\subsection{Problem in transmission: Critical coupling condition in thin aluminum plates}

Thin plates may be defined as flat elements having two dimensions (length, $a$, and breadth, $b$ ) that are large in comparison to the remaining one (depth, $L$ ) [82]. Plates are bounded either by straight or curved boundaries and their bending properties depend greatly on its thickness as compared with their other dimensions, as well as on the material properties of the plate [83].

In elastic solids shaped into plate-like form, three types of waves occur: transverse shear, longitudinal, and flexural waves. Of these waves, flexural waves are by far the most important in the field of noise and vibration control because of their direct contribution to sound radiation [84].

Quantitatively, Ventsel [85] classified plates in three groups attending to the ratio between the length, $a$, and the thickness, $L$. Since we are considering square plates, $a=b$. The three groups are:

1. Thick plates $(a / L \leq 10)$. The analysis of such bodies includes all the components of stresses, strains and displacements for solid bodies using the general equations of three-dimensional elasticity.

2. Thin plates $(a / L \geq 100)$. These plates are referred to as membranes and the effects of their flexural rigidity can be neglected. Membranes carry the lateral loads by axial tensile forces $N$ (and shear forces) acting in the plate middle surface. These forces are called membrane forces. They produce projection on a vertical axis and thus balance a lateral load applied to the plate-membrane.

3. Stiff plates $(10 \leq a / L \leq 100)$. Depending on the value of the ratio $a / L$, the ratio of the maximum deflection of the plate to its thickness, the part of flexural and membrane forces in these plates may be different.

Here the attention is paid to the case of thin plates. The interest in the study of propagation of flexural waves in thin plates has increased in the recent years, as they present some interesting properties for sound absorption and the control of wave propagation. The propagation of flexural waves in thin plates presents the peculiarity that it can be controlled by means of thickness variations, as was theoretically studied by Krylov [86]. When there is open flow through the structure, it is relatively straightforward to extract effective properties experimentally due to the relatively low acoustic impedance [87]. Some studies have been performed about sound absorption of multilayer perforated panel 


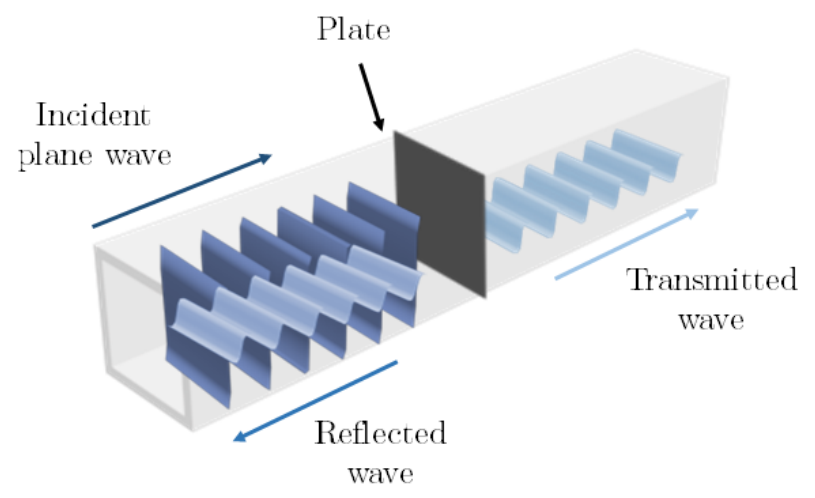

Figure 3.5: Model of a system composed by a single plate placed in an impedance tube.

systems [88-91] and plates [92] with the aim of obtaining higher absorption both in magnitude and in a broad frequency range. The thickness of the plate, $L$, is considered to be much smaller than the wavelength of the incoming waves, $\lambda(\lambda>>L)$.

Most of the results presented in the acoustic analysis of the works mentioned above are based upon linear models. Using plates, it is possible to achieve high absorption, especially at some frequencies at which the natural modes of the plate are excited. The work performed by Lee et al. [88] shows the increase of the absorption coefficient at the resonance frequency of the resonating element.

In this Chapter we study the critical coupling conditions to maximize the sound absorption in a system composed by a thin aluminum square plate. We consider a system composed of this plate clamped into an impedance tube (see Figure 3.5). Since all its sides are constrained by the walls of the tube, clamped boundary conditions apply to all the contour of the plate.

The range of frequencies in which the study is carried out must be below the cut-off frequency of the tube, determined in order to ensure the propagation of plane waves and that no transverse modes appear in the tube. Since the waves propagate into the tube in one direction (in the $x$-axis), only flexural modes in the plate are considered and, as a consequence, the problem can be studied as a 1-D problem in the $x$ direction (see Figure 3.6). 


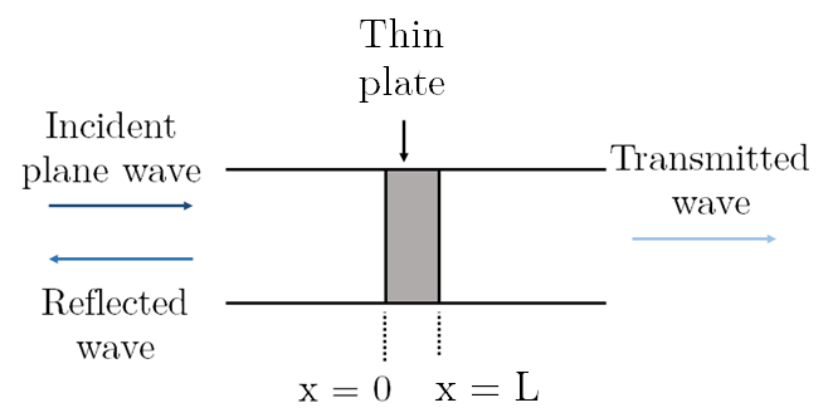

Figure 3.6: 1-D scheme of the problem in transmission.

The system can be analyzed by using the transfer matrix between its two faces, $\mathbf{M}$, extending from $x=0$ to thickness $x=L$, as following the procedure exposed in Section 3.1.1.

The clamped plate is a locally resonant element in series with the waveguide. As $L$ is much smaller than $\lambda(L<<\lambda)$, it is possible to consider the plate as a punctual resonator in the $x$ direction. Defining the pressure at both sides as $p(0)$ and $p(L)$ and the corresponding particle velocity as $v(0)$ and $v(L)$, the effect of the plate on the acoustic field is twofold: $(i)$ the velocity is the same at both sides of the plate due to the continuity boundary conditions $(v(0)=v(L))$; (ii) there is a pressure drop between each side due to the presence of the plate, and it is possible to relate it at both sides of the punctual resonator by using its acoustic impedance as [78].

$$
Z_{p}=\frac{\Delta p}{v}=\frac{p(0)-p(L)}{v(L)} .
$$

Relating Eq. (3.21) to the general equation of the transfer matrix, (Eq. (3.3)), it is possible to obtain the transfer matrix of a plate, $M_{p}$ as

$$
M_{p}=\left[\begin{array}{cc}
1 & Z_{p} \\
0 & 1
\end{array}\right]
$$

In order to obtain $Z_{p}$, clamped boundary conditions are applied to all the boundaries of the plate, as they are fixed by the walls of the waveguide. This means that both the displacement, $u$, and the velocity, $v$, of the boundaries are equal to zero. 
Keeping the boundary conditions in mind, the impedance of the plate can be calculated as follows [93],

$$
Z_{p}=\frac{\Delta p}{v}=\left(\sum_{m=1}^{M} \sum_{n=1}^{N} \frac{\varepsilon_{m n}^{2}}{\eta_{m n} Z_{m n}}\right)^{-1}
$$

where,

$$
\begin{gathered}
\varepsilon_{m n}=\int_{0}^{b} \int_{0}^{a} X(x) Y(y) d x d y, \\
\eta_{m n}=\int_{0}^{b} \int_{0}^{a} X(x)^{2} Y(y)^{2} d x d y,
\end{gathered}
$$

being $\varepsilon_{m n}$ and $\eta_{m n}$ eigenfunction parameters for a clamped-clamped beam, and

$$
\begin{gathered}
X_{m}(x)=\cosh \left(\frac{\epsilon_{m} x}{a}\right)-\cos \left(\frac{\epsilon_{m} x}{a}\right)-\alpha_{m}\left[\sinh \left(\frac{\epsilon_{m} x}{a}\right)-\sin \left(\frac{\epsilon_{m} x}{a}\right)\right], \\
Y_{n}(y)=\cosh \left(\frac{\epsilon_{n} y}{b}\right)-\cos \left(\frac{\epsilon_{n} y}{b}\right)-\alpha_{n}\left[\sinh \left(\frac{\epsilon_{n} y}{b}\right)-\sin \left(\frac{\epsilon_{n} y}{b}\right)\right],
\end{gathered}
$$

being $X_{m}(x)$ and $Y_{n}(y)$ the fundamental mode shapes of beams having the boundary conditions of the plate.

Note that the values of $\epsilon_{m}, \epsilon_{n}, \alpha_{m}$ and $\alpha_{n}$ are tabulated at [94] (see Table 3.1):

\begin{tabular}{|c|c|c|}
\hline $\mathbf{m}, \mathbf{n}$ & $\alpha_{\mathbf{m}}, \alpha_{\mathbf{n}}$ & $\epsilon_{\mathbf{m}}, \epsilon_{\mathbf{n}}$ \\
\hline 1 & 0.98250222 & 4.7300408 \\
\hline 2 & 1.00077731 & 7.8532046 \\
\hline 3 & 0.99996645 & 10.9956078 \\
\hline 4 & 1.00000145 & 14.1371655 \\
\hline 5 & 0.99999994 & 17.2787596 \\
\hline 6 & 1.00000000 & 20.4203522 \\
\hline$r>6$ & 1.00000000 & $(2 r+1) \pi / 2$ \\
\hline
\end{tabular}

Table 3.1: Eigenfunction parameters for a clamped-clamped beam (extracted from [94]) 
In Eq. (3.23) $Z_{m n}$ is defined as

$$
Z_{m n}=\rho_{v} \frac{i\left(\omega^{2}-\omega_{m n}^{2}\right)}{\omega}
$$

and represents the lossless modal impedance of the $(m, n)$-th mode, being $\rho_{v}$ the volumetric density and $\omega_{m n}$ the resonance frequency of the $(m, n)$-th mode of the plate.

If the effect of the modal damping on the plate are considered, $Z_{m n}$ includes the flexural loss factor, $\xi$. Loss factor usually refers to the conversion of energy into heat [95], in this case produced by flexural modes in the plate. $Z_{m n}$ can be rewritten as

$$
Z_{m n}=\rho_{v} \frac{\xi \omega_{m n} \omega+i\left(\omega^{2}-\omega_{m n}^{2}\right)}{\omega}
$$

It is important to note that in this Chapter only the mode $(1,1)$, corresponding to the fundamental mode of the plate (also known as "piston-like mode"), is considered. Higher order modes are out of the range of frequencies of interest, since we want to focus in this Section in the study of the critical coupling associated to the fundamental mode. However, it is important to note that the procedure for higher order modes is analogous. The equation that allows to calculate the frequency of this mode, Eq. (3.30), is presented by Leissa [94], and is an approximation for the flexural modes of square plates when $m=n$. Thus, the $(m, m)$ flexural mode can be analytically obtained as follows,

$$
\omega_{m}=2\left(m+\frac{1}{3}\right)^{2} \frac{\pi^{2}}{a^{2}} \sqrt{\frac{D}{\rho_{s}}},
$$

where, $a$ is the size of the plate, $\rho_{s}=\rho_{v} h$ is the surface density, and $D=$ $E h^{3} / 12\left(1-\nu^{2}\right)$ the bending stiffness. Since the plate is made of aluminum, we consider the following material's properties: Young's modulus $E=69 \mathrm{GPa}$, density $\rho=2712 \mathrm{~kg} / \mathrm{m}^{3}$ and Poisson's ratio $\nu=0.334$. The thickness of the plate is considered to be $h=0.5 \mathrm{~mm}$.

The reflection and transmission coefficients can be directly calculated from the elements of the transfer matrix, as it has been already deduced in Eqs. (3.10) and (3.11). 
Since both properties are fulfilled in this system, the reflection and transmission coefficients are given in a more simplified form by

$$
\begin{aligned}
& T=\frac{2}{2+Z_{p} / Z_{0}}, \\
& R=\frac{Z_{p} / Z_{0}}{2+Z_{p} / Z_{0}},
\end{aligned}
$$

being $Z_{0}$ the acoustic impedance of air.

The sound absorption coefficient is readily obtained from these coefficients as

$$
\alpha=1-|T|^{2}-|R|^{2} .
$$

\subsubsection{Lossless case}

When no losses are considered in the system, $\xi=0$ in Eq. (3.29), the energy that is not reflected back due to the presence of the plate is transmitted, since no absorption can be produced. Figure 3.7 shows acoustic impedance and the transmission, reflection and absorption coefficients with respect to the frequency. The frequencies are normalized to the resonance frequency of the plate.

As it can be observed in Figure 3.7a), at the normalized frequency $\omega / \omega_{\text {res }}=1$ the imaginary part of the impedance, $\operatorname{Im}\left(Z_{p}\right)$, is equal to zero, fulfilling the resonant condition. At the resonance frequency $Z_{p}=0$ and, as a consequence, there is perfect transmission $(T=1)$ and a minimum of reflection $(R=0)$ as follows from Eqs. (3.31) and (3.32). Figure 3.7b) shows this effect.

The eigenvalues of the scattering matrix are strongly dependent of the impedance of the obstacle and, as a consequence of this, they depend on the frequency.

Figure 3.8 shows this dependence of the eigenvalues of the scattering matrix with the frequency for the lossless and the lossy case. In the lossless case, given the energy conservation, both eigenvalues are equal to 1 at the resonance frequency.

In the lossy case, part of the energy is also absorbed. The maximum value of one-sided absorption appears at the resonance frequency and has a value of 0.5. 
a)

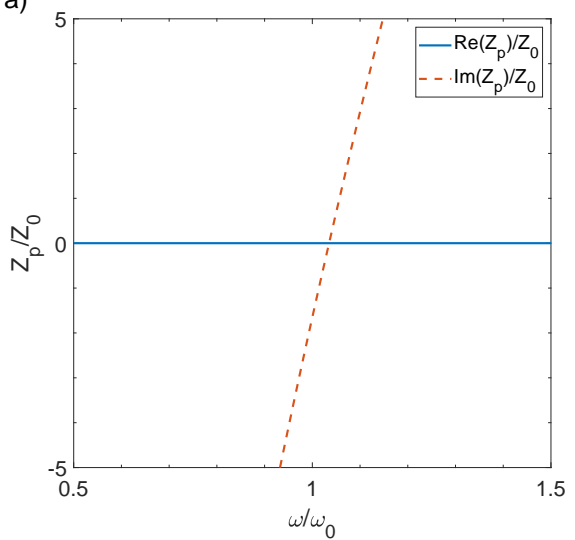

b)

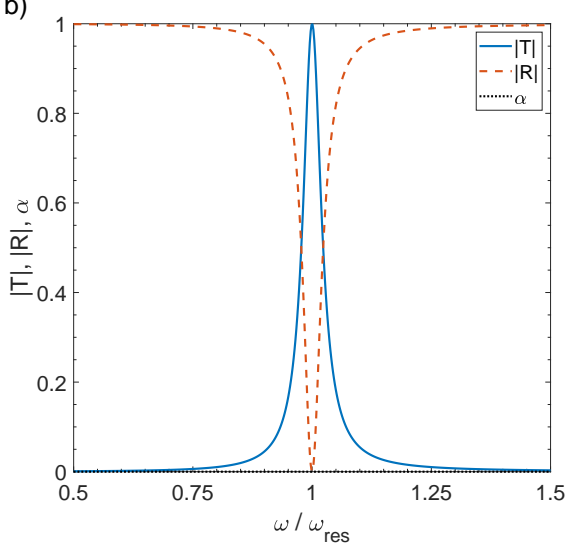

Figure 3.7: a) Real (solid blue line) and imaginary (dashed red line) part of the impedance of the plate. b) Transmission (solid blue line), reflection (dashed red line) and absorption (dotted black line) coefficients for a lossless case.

a)

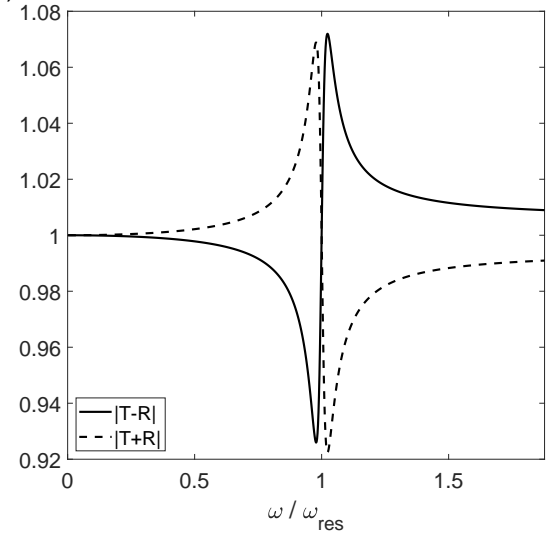

b)

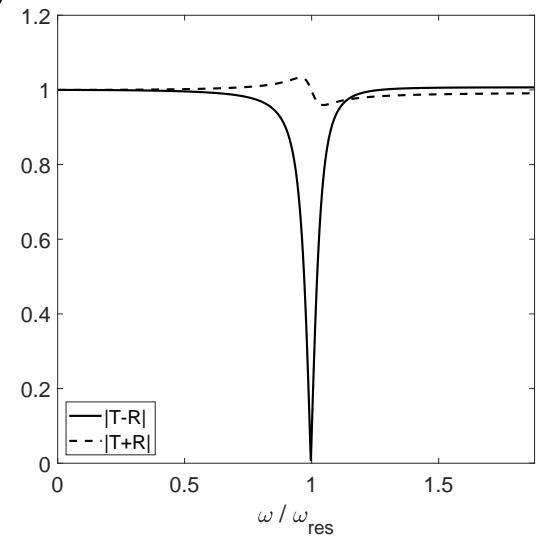

Figure 3.8: Eigenvalues of the system: a) Lossless case, b) Lossy case. 
At this frequency, one of the two eigenvalues is null at the resonance frequency, $\lambda_{1}\left(\omega_{\text {res }}\right)=0$, while the other one has a value of $1, \lambda_{2}\left(\omega_{\text {res }}\right)=1$. The obstacle is transparent to anti-symmetric incident waves, $\alpha_{a}=0$. Thus $\alpha=\alpha_{s} / 2 \leq$ $1 / 2$ [79]. As a consequence, the maximum absorption coefficient that can be achieved with a single resonating element in transmission is $\alpha_{\max }=0.5$. In a system with a single resonator only one mode can be excited. In this case, the eigenvalue with value equal to 0 is the one related to the efficient absorption of energy, while the other one remains almost invariable and has practically no influence in this phenomenon [78].

\subsubsection{Lossy case}

The absorption is produced due to energy losses present in the system associated to different dissipative mechanisms, which are caused by different parts of the system [96]. On the one hand, viscothermal losses are present due to the friction of the sound wave with the walls of the waveguide. Thus, this type of losses are inherent to the propagation of the sound wave into the duct and depend on its design parameters, such as the geometry, the cross-section and the roughness of the internal wall, among others. On the other hand, viscoelastic losses are present in the plate.

Viscoelastic losses in the plate are introduced in the model by considering the flexural loss factor, $\xi$, in the equation of the modal impedance (Eq. (3.29)). In this case, we are going to use aluminum as an example. The characteristic flexural loss factor in aluminum varies from $\xi=3 \cdot 10^{-5}$ to $\xi=10^{-4}$ [95]. Due to the low value of the intrinsic losses of the aluminum plate, it has a very low influence in the sound absorption by itself. As can be observed in Figure 3.9 , one single aluminum plate with the characteristics mentioned above has an absorption of $0.4 \%$.

This low influence leads to the need of implementing new solutions in order to add losses to the system by increasing the viscoelastic losses with the goal of reaching the critical coupling conditions and maximize the sound absorption. In order to increase the viscoelastic losses, it is possible to consider the inclusion of an absorbing layer in contact with the plate. The addition of an absorbing layer results in a composite (plate + absorbing material) that can be studied as a single layer with effective properties by using the Ross-Kerwin-Ungar (RKU) model [97]. This model was developed for calculating the damping of flexural vibrations by alternate viscoelastic and elastic layers. By using the RKU model, it is possible to describe the system plate-absorptive layer as a single composite with effective mechanical properties. 


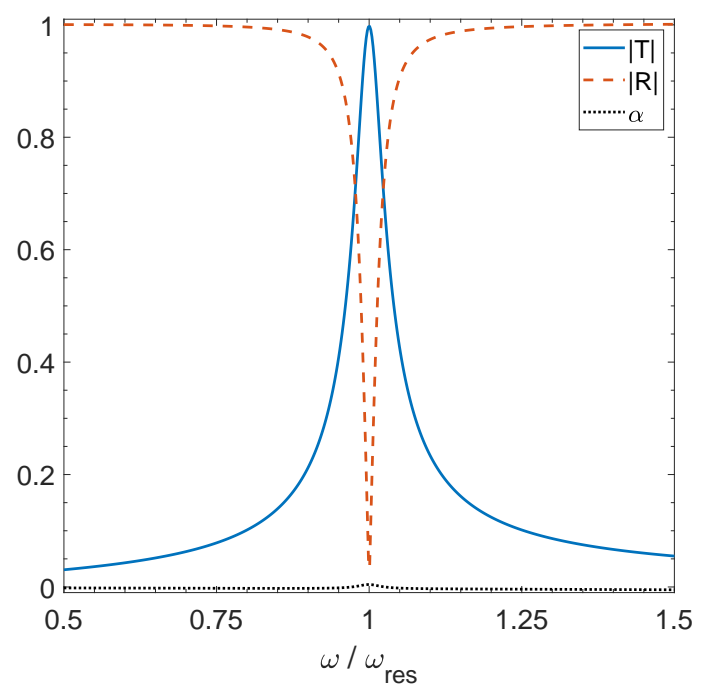

Figure 3.9: Transmission (solid blue line), reflection (dashed red line) and absorption (dotted black line) coefficients for a lossy case, considering the intrinsic losses of the aluminum plate.

This model states that the wavenumber of the composite material is

$$
k_{c}^{4}=\frac{12 \omega^{2} \rho_{p}\left(1-\nu_{p}^{2}\right)}{E_{p} h_{p}^{2}}\left[\frac{1+\rho_{r} h_{r}}{\left(1-i \eta_{p}\right)+\left(1-i \eta_{l}\right) h_{r} E_{r} \beta}\right]
$$

where the subscripts $p$ and $l$ stand for the parameters of the plate and the absorbing layer, respectively, $E$ is the Young's modulus, and $\rho_{v}$ is the volumetric density. $\rho_{r}=\rho_{l} / \rho_{p}, h_{r}=h_{l} / h_{p}, E_{r}=E_{l} / E_{p}$ and $\beta=3+6 h_{r}+4 h_{r}^{2}$ being $h$ the thickness.

The thickness of the composite, $h_{c}$, is simply the total thickness of the two layers,

$$
h_{c}=h_{p}+h_{l}
$$

while the density is the volume average of the densities of the two materials, 


$$
\rho_{c}=\frac{\rho_{p} h_{p}+\rho_{l} h_{l}}{h_{c}}
$$

It is assumed that the Poisson's ratio of the composite, $\nu_{c}$, is the same as that of the plate

$$
\nu_{c}=\nu_{p}
$$

The flexural rigidity is obtained as

$$
D_{c}=\frac{\omega^{2} \rho_{c} h_{c}}{k_{c}^{4}}=\frac{h_{p}^{3}}{12\left(1-\nu_{p}^{2}\right)} E_{p}\left[\left(1+h_{r} E_{r} \alpha\right)-i\left(\eta_{p}+\eta_{l} h_{r} E_{r} \beta\right)\right] .
$$

Finally, knowing that $E_{c}=12 D_{c}\left(1-\nu_{c}^{2}\right) / h_{c}^{3}$, the Young's modulus of the composite is obtained and its loss factor is found as

$$
\xi_{c}=\frac{\Im\left\{E_{c}\right\}}{\Re\left\{E_{c}\right\}}=\frac{\xi_{p}+\xi_{l} E_{r} \beta}{1+h_{r} E_{r} \beta} .
$$

By following the equations governing the RKU model, a particular case is developed from now on. An absorptive layer made of moulded polystyrene has been added in contact with the aluminum plate. This material has a Young's modulus $E_{l}=7.8 \mathrm{GPa}$, density $\rho_{l}=1400 \mathrm{~kg} / \mathrm{m}^{3}$, Poisson ratio $\nu_{l}=0.34$ and loss factor $\xi_{l}=0.1$.

To achieve the right amount of losses in the system, it is possible to study the effect of the loss factor on the sound absorption coefficient. The relationship between the loss factor and the sound absorption is represented graphically (see Figure 3.10). One can observe that there is an optimum value in which the maximum absorption is produced. As it can be seen in the figure, there is an exact amount of losses (in this case $\xi_{c}\left(\alpha_{\max }\right)=0.0447$ ) for which the maximum absorption is achieved.

A loss factor of the composite of $\xi_{c}\left(\alpha_{\max }\right)=0.0447$ is necessary to balance the leakage and losses in the system, as it can be extracted from Figure 3.10), and marked with the dashed line. By using the equations of the RKU model, we can calculate the thickness of the absorptive layer, that must be $h_{l}=225 \mu \mathrm{m}$ to have critical coupling. Note that the loss factor of the material composing the absorptive layer is three orders of magnitude greater than that of the 


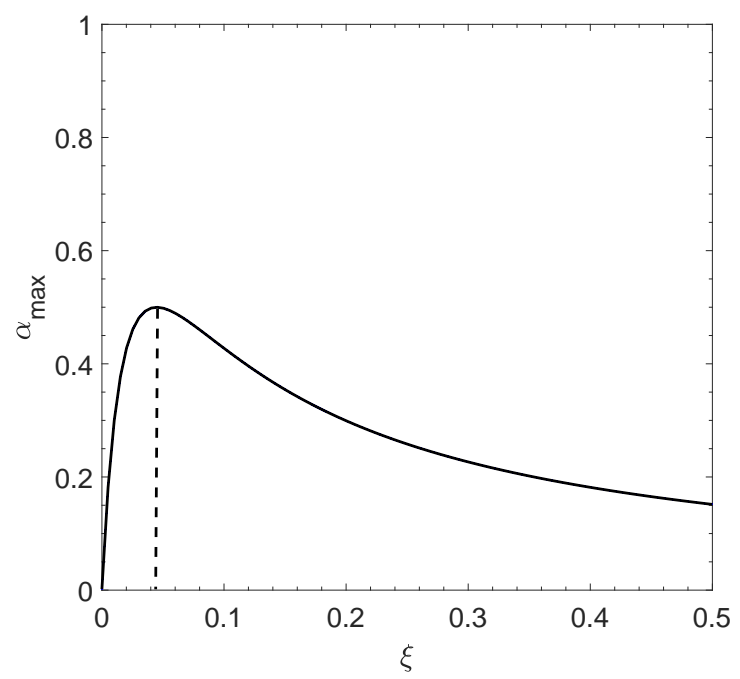

Figure 3.10: Relation between the loss factor and the maximum sound absorption coefficient.

aluminum ( $\xi=0.1$ versus $\xi=10^{-4}$, respectively). The increase of the loss factor of the composite, $\xi_{c}$, introduced by the absorptive material up to the value of $\xi_{c}\left(\alpha_{\max }\right)$ is fundamental for obtaining the sound absorption coefficient of the system.

The effective properties of the composite are Young's modulus $E_{c}=23.7 \mathrm{GPa}$, density $\rho_{c}=2154 \mathrm{~kg} / \mathrm{m}^{3}$, Poisson ratio $\nu_{c}=0.34$ and loss factor $\xi_{c}=0.0447$. Since the required loss factor for the composite has been achieved, an increase of the sound absorption coefficient up to $\alpha=0.5$ can be observed as a result (see Figure 3.11).

The optimum absorption of $\alpha_{\max }=0.5$ found in Figure 3.11 is characteristic of systems with open resonating elements fulfilling the critical coupling condition [98]. In the case of a symmetric resonator and one-sided excitation (as considered in this work) critical coupling leads to a 0.5 of absorption [53, 79, 99].

If the plate is considered as a punctual symmetric resonator due to the fact that the thickness, $L$, is much smaller than the wavelength, $\lambda$, the imposition of the continuity of the wave-field at this point yields $1+R=T$ [79]. The onewave transmission and reflection of a scatterer is independent of the amplitude 


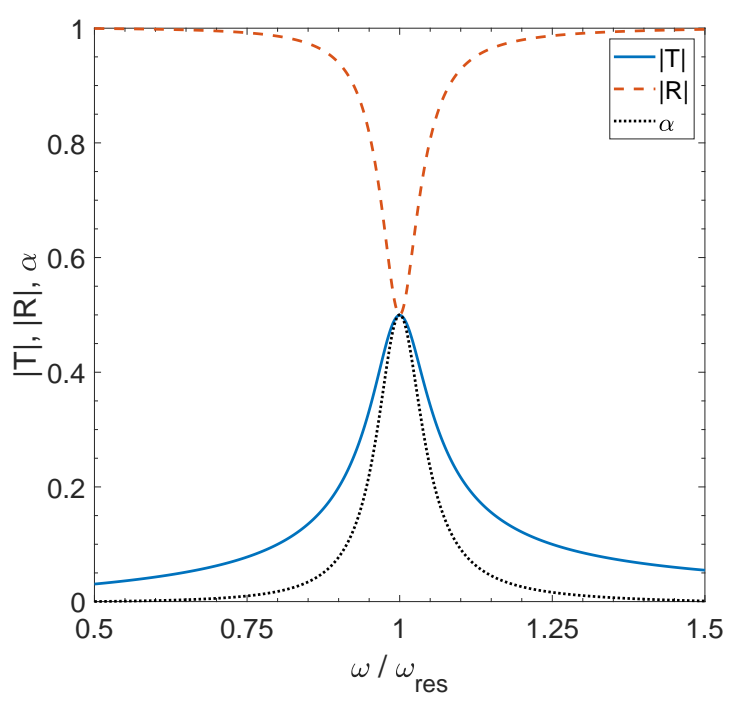

Figure 3.11: Transmission (solid blue line), reflection (dashed red line) and absorption (dotted black line) coefficients for a lossy case presenting critical coupling.

of the incident wave in the linear acoustic regime, so they can be regarded as intrinsic of the scatterer. Combined with the constraint on $R$ and $T$ due to the point symmetry, and that $R=-T$, the magnitudes of $R$ and $T$ must be 0.5 each for perfect absorption. This leads to a maximum sound absorption coefficient $\left(\alpha=1-|T|^{2}-|R|^{2}\right)$ of 0.5 under the incident wave [100].

In order to confirm the result given in Figures 3.10 and 3.11, a numerical model using the Finite Element Method (FEM) has been implemented by considering the same configuration. The geometry used in the model can be observed in Figure 3.12 .

The model solves the Helmholtz equation in the domains corresponding to air in the frequency domain for a given range of frequencies. Acoustic waves propagate in a fluid (air) with harmonic variations of the pressure field. This harmonic variation is given by $e^{i \omega t}$ using the $+i \omega$ convention. The plate is modeled as a linear elastic material with the effective properties resulting from the equations of the RKU model. Fixed boundary conditions have been considered in all the boundaries of the plate, since it is clamped to the walls of the tube. Thus, the displacement of these boundaries and the curvature of the edges are zero in all directions. 


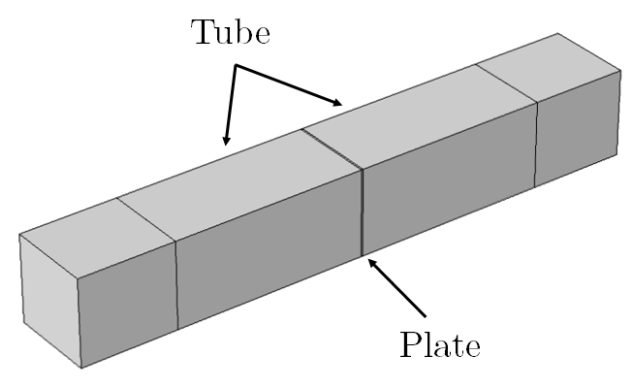

Figure 3.12: 3 -D numerical model implemented to solve the problem by using the FEM.

Figure 3.13 shows the comparison between the absorption coefficient obtained by using the TMM and the FEM. The difference of the resonance frequency (maximum of the peak of sound absorption) between both methods is $0.4 \mathrm{~Hz}$, so the agreement between both models is good. The maximum absorption at the resonance frequency of the plate occurs due to the critical coupling.

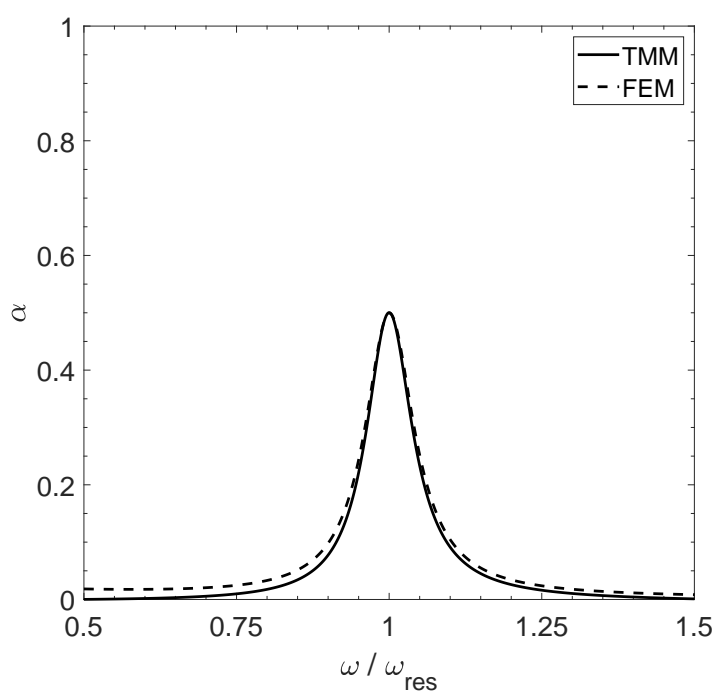

Figure 3.13: Absorption coefficient calculated for the lossy case by means of the TMM (solid black line) and the FEM (dashed black line). 


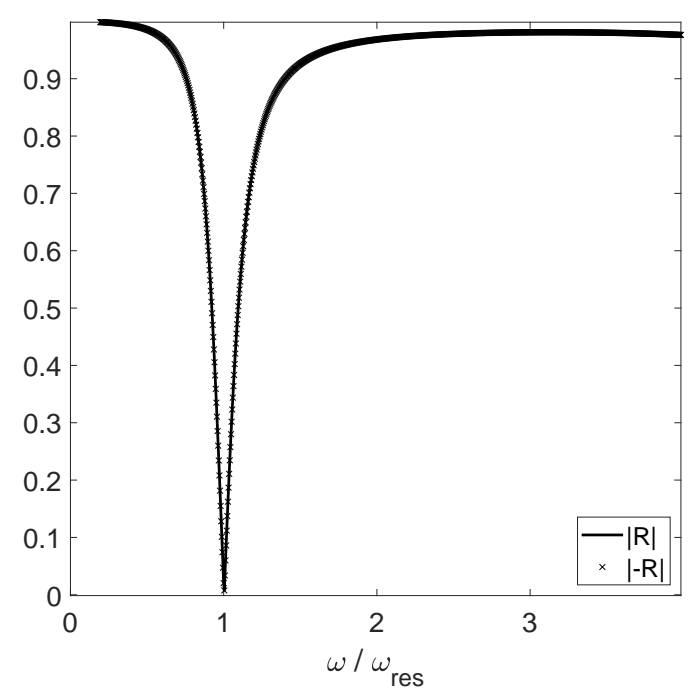

Figure 3.14: Eigenvalues of the system.

\subsection{Problem in reflection: Critical coupling condition in Helmholtz resonators}

As opposite to the case in transmission, in purely reflecting systems the energy can be trapped by using either symmetric or antisymmetric modes [58]. When these modes (either symmetric, antisymmetric or both) are critically coupled, perfect absorption of energy is produced. At the resonance frequency of the obstacle, the two eigenvalues of the scattering matrix are null, meaning that both $\lambda$ are used to dissipate energy. As a consequence, perfect absorption $(\alpha=1)$ can be produced. Figure 3.14 shows the eigenvalues of the scattering matrix of the problem in reflection dependent with the frequency.

A Helmholtz resonator (HR) is a lumped acoustic system that consists of a rigid-walled cavity of a given volume with a neck [32]. The volume of air confined in the cavity behaves as a spring with a given compliance and induces the vibration of the air in and near the neck. The air in the neck acts as an added mass to the one confined in the cavity of the HR. These systems have been widely studied in the field of Acoustics due to their interesting properties in sound control [101]. 
The classical analysis of a HR predicts that the resonance frequency, $\omega_{\text {res }}$, is independent of the shape of the cavity due to the assumption that all dimensions are small compared to the wavelength [102]. The theoretical resonance frequency of HRs, in which the dimension in the $z$ direction is considered as infinite, can be predicted by using Eq. (3.40).

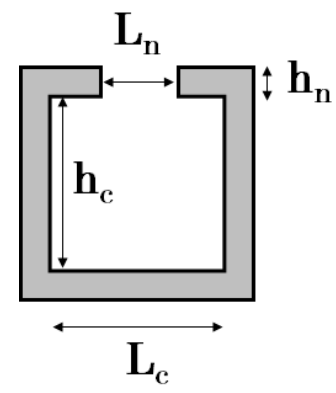

Figure 3.15: Geometrical parameters of a Helmholtz resonator.

For the case of a HR (see Figure 3.15), as the ones that are studied in this Thesis, the dimension transverse to the direction of propagation of the sound waves is considered as infinite and plays no role in the behavior of the system in this case. Thus, the resonance frequency of a $2 \mathrm{D}$ HR is expressed as follows:

$$
\omega_{\text {res }}=c_{0} \sqrt{\frac{L_{n}}{h_{n} L_{c} h_{c}}},
$$

where $c_{0}$ is the sound speed in air, $L_{n}$ and $h_{n}$ are, respectively, the length and the height of the neck of the resonator, and $L_{c}$ and $h_{c}$ are the length and height of the cavity of the HR (see Figure 3.15).

In order to study the critical coupling conditions in a problem in reflection, the following case of study has been considered: a single Helmholtz resonator in contact with the rigid backing, meaning that no air cavity is placed between these two elements. The rigid backing precludes the possibility of a transmitted wave beyond it and no transmitted field is considered. The system is placed into an impedance tube, and a slit formed between the upper part of the HR and the impedance tube is also considered (Figure 3.16). In this case, the length of the cavity behind the HR, $L$, is considered as $L=0$. 


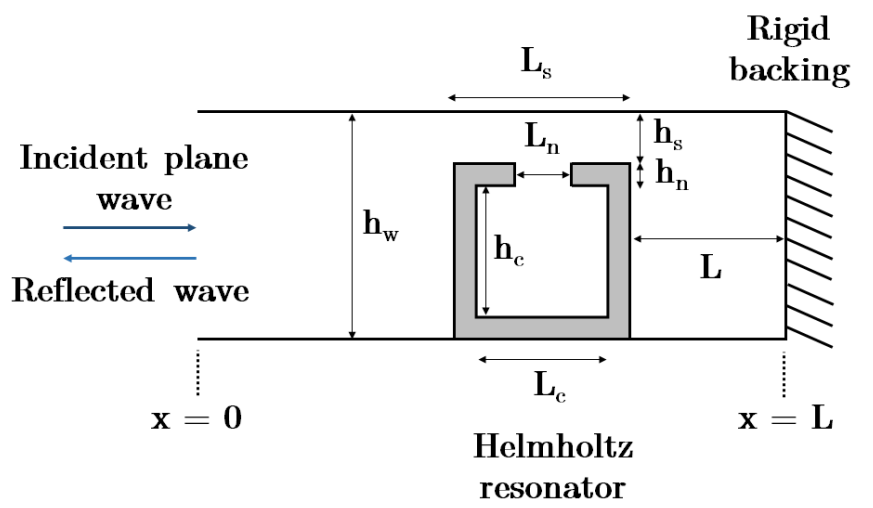

Figure 3.16: 1-D scheme of a system composed of a single Helmholtz resonator.

The study of this case is interesting because it will allow the understanding of the phenomenology that takes place in systems composed of HRs and verify the models by reducing the problem to its simplest variant.

In order to study the individual contribution of each subsystem, composed by a HR and the cavity placed behind it, to the distribution of the pressure field, the complete system can be decomposed in each of the elements and a transfer matrix can be defined for each of them as in [59]. For the case of a single HR embedded in a tube, the transfer matrix can be obtained by assembling the matrices representing each element of the system:

$$
M=\left[\begin{array}{ll}
M_{11} & M_{12} \\
M_{21} & M_{22}
\end{array}\right]=M_{\Delta_{s l i t}} M_{s} M_{H R} M_{s} M_{\Delta_{s l i t}} M_{w} .
$$

$M_{\Delta l_{s i t}}$ represents the transfer matrix corresponding to the correction due to the change of section between the main tube and the Helmholtz resonator (if there is not change of section, this matrix is the identity matrix). It can be defined as

$$
\mathbf{M}_{\Delta l_{s l i t}}=\left[\begin{array}{cc}
1 & Z_{\Delta l_{s l i t}} \\
0 & 1
\end{array}\right]
$$

The transmission matrix for the slit, $M_{s}$, represents the propagation of waves through the slit along a distance $L_{s} / 2$. It reads as 


$$
M_{s}=\left[\begin{array}{cc}
\cos \left(k_{s} \frac{L_{s}}{2}\right) & i Z_{s} \sin \left(k_{s} \frac{L_{s}}{2}\right) \\
\frac{i}{Z_{s}} \sin \left(k_{s} \frac{L_{s}}{2}\right) & \cos \left(k_{s} \frac{L_{s}}{2}\right)
\end{array}\right],
$$

where $Z_{s}$ is the acoustic impedance and $k_{s}$ is the wavenumber in the slit.

The transmission matrix for each resonator, $M_{H R}$, is defined as

$$
M_{H R}=\left[\begin{array}{cc}
1 & 0 \\
\frac{1}{Z_{H R}} & 1
\end{array}\right]
$$

being $Z_{H R}$ the acoustic impedance of the Helmholtz resonator (this parameter is defined in Eq. (3.52)).

The transmission matrix for the waveguide and the cavities placed behind the HRs, $M_{w}$, is defined later in the following form

$$
M_{w}=\left[\begin{array}{cc}
\cos \left(k_{w} L_{w}\right) & i Z_{0} \sin \left(k_{w} L_{w}\right) \\
\frac{i}{Z_{0}} \sin \left(k_{w} L_{w}\right) & \cos \left(k_{w} L_{w}\right)
\end{array}\right] .
$$

The reflection coefficient can be obtained from the terms of the transfer matrix (Eq. (3.41)) as in Ref. [77],

$$
R=\frac{M_{11}-Z_{0} M_{21}}{M_{11}+Z_{0} M_{21}}
$$

where $Z_{0}$ is the acoustic impedance of air, and $M_{i j}$ refers to the term in the row $i$ and column $j$ of the M-matrix.

The absorption coefficient is directly calculated as,

$$
\alpha=1-|R|^{2}
$$




\subsubsection{Resonator impedance and end corrections}

The acoustic impedance of the Helmholtz resonator [58], including the length correction due to the radiation of the neck, is derived from the matrices of the different elements composing the HR.

$$
\left[\begin{array}{l}
p \\
v
\end{array}\right]_{x=0}=M_{n} M_{\Delta l_{n}} M_{c}\left[\begin{array}{l}
p \\
v
\end{array}\right]_{x=L}
$$

The transfer matrix corresponding to the neck, $M_{n}$, and the one of the cavity, $M_{c}$, of the HR read respectively as

$$
\begin{gathered}
M_{n}=\left[\begin{array}{cc}
\cos \left(k_{n} L_{n}\right) & i Z_{n} \sin \left(k_{n} L_{n}\right) \\
\frac{i}{Z_{n}} \sin \left(k_{n} L_{n}\right) & \cos \left(k_{n} L_{n}\right)
\end{array}\right], \\
M_{c}=\left[\begin{array}{cc}
\cos \left(k_{c} L_{c}\right) & i Z_{c} \sin \left(k_{c} L_{c}\right) \\
\frac{i}{Z_{c}} \sin \left(k_{c} L_{c}\right) & \cos \left(k_{c} L_{c}\right)
\end{array}\right] .
\end{gathered}
$$

The transfer matrix corresponding to the length correction in the neck, $M_{\Delta l_{n}}$, is

$$
M_{\Delta l_{n}}=\left[\begin{array}{cc}
1 & i Z_{n} k_{n} \Delta l_{n} \\
0 & 1
\end{array}\right]
$$

$Z_{H R}$ can be obtained from the quotient of $p$ and $v$ in Eq. (3.48), and written as

$$
\begin{array}{r}
Z_{H R}=-i \frac{\cos \left(k_{n} L_{n}\right) \cos \left(k_{c} L_{c}\right)-Z_{n} k_{n} \Delta l \cos \left(k_{n} L_{n}\right) \sin \left(k_{c} L_{c}\right) / Z_{c}-}{\sin \left(k_{n} L_{n}\right) \cos \left(k_{c} L_{c}\right) / Z_{n}-k_{n} \Delta l \sin \left(k_{n} L_{n}\right) \sin \left(k_{c} L_{c}\right) / Z_{c}+} \\
\frac{-Z_{n} \sin \left(k_{n} L_{n}\right) \sin \left(k_{c} L_{c}\right) / Z_{c}}{+\cos \left(k_{n} L_{n}\right) \sin \left(k_{c} L_{c}\right) / Z_{c}}
\end{array}
$$

where the correction length is induced from the addition of two correction lengths $\Delta l=\Delta l_{1}+\Delta l_{2}$ as

$$
\Delta l_{1}=0.82\left[1-1.35 \frac{L_{n}}{L_{c}}+0.31\left(\frac{L_{n}}{L_{c}}\right)^{3}\right] L_{n},
$$




$$
\Delta l_{2}=0.82\left[1-0.235 \frac{L_{n}}{L_{s}}-1.32\left(\frac{L_{n}}{L_{s}}\right)^{2}+1.54\left(\frac{L_{n}}{L_{s}}\right)^{3}-0.86\left(\frac{L_{n}}{L_{s}}\right)^{4}\right] L_{n}
$$

$\Delta l_{1}$, is the length correction given by the pressure radiation at the discontinuity from the neck duct to the cavity of the Helmholtz resonator [103], and $\Delta l_{2}$, comes from the radiation at the discontinuity from the neck to the slit [104].

The radiation from the slits to the free air requires a different correction model. The radiation correction for the slit $\Delta l_{\text {slit }}$ can be expressed as [105],

$$
\Delta l_{\text {slit }}=h \phi_{t} \sum_{n=1}^{\infty} \frac{\sin ^{2}\left(n \pi \phi_{t}\right)}{\left(n \pi \phi_{t}\right)^{3}}
$$

being, $\phi_{t}=h_{s} / h_{t}$ the porosity. Note that, for $0.1 \leq \phi_{t} \leq 0.7$, this expression reduces to $\Delta l_{\text {slit }} \approx-\sqrt{2} \ln \left[\sin \left(\pi \phi_{t} / 2\right)\right] / \pi[58]$.

\subsubsection{Viscothermal losses}

The viscothermal losses in the system are considered in both the resonators and the slit by using effective complex and frequency dependent parameters [106].

The effective parameters in the slit, neck and cavity of the HR, considering only plane waves propagating inside, are expressed as

$$
\begin{gathered}
\rho_{i}=\rho_{0}\left[1-\frac{\tanh \left(\frac{h}{2} G_{\rho}\right)}{\frac{h}{2} G_{\rho}}\right]^{-1}, \\
\kappa_{i}=\kappa_{0}\left[1+(\gamma-1) \frac{\tanh \left(\frac{h}{2} G_{\kappa}\right)}{\frac{h}{2} G_{\kappa}}\right]^{-1},
\end{gathered}
$$

with $G_{\rho}=\sqrt{i \omega \rho_{0} / \mu}$ and $G_{\kappa}=\sqrt{i \omega \operatorname{Pr} \rho_{0} / \mu}$, and where $\gamma=1.4$ is the specific heat ratio of air, $P_{0}=101325 \mathrm{~Pa}$ is the atmospheric pressure, $\operatorname{Pr}=0.71$ is the Prandtl number, $\mu=1.839 \cdot 10^{-5} \mathrm{~kg} / \mathrm{m} \cdot \mathrm{s}$ the dynamic viscosity, $\rho_{0}=1.213$ $\mathrm{kg} / \mathrm{m}^{3}$ the air density and $\kappa_{0}=\gamma P_{0}$ the air bulk modulus. The subscript $i$ can 
be considered as $s, c$ or $n$ if the calculations are referred to the slit, the cavity or the neck of the HR, respectively.

The effective wavenumber and the characteristic impedance can be calculated as

$$
\begin{gathered}
k_{i}=\omega \sqrt{\frac{\rho_{i}}{\kappa_{i}}}, \\
Z_{i}=\sqrt{\kappa_{i} / \rho_{i}} / S_{i},
\end{gathered}
$$

with $S_{i}=h_{i} L_{i}$ being the cross-section.

\subsection{Conclusions}

The conditions for critical coupling have been studied in this chapter in two different configurations: a thin aluminum square plate in contact with an absorptive layer (problem in transmission) and a Helmholtz resonator backed by a rigid ending (problem in reflection).

By considering the equations governing the TMM and tuning the losses of both systems, it is possible to maximize the sound absorption in both systems. It has also been shown how a lack or excess of losses in the system break the critical coupling condition, giving as a consequence a decrease in the sound absorption coefficient.

In the problem in transmission, when the system is composed by an elastic plate, it is possible to tune the viscoelastic losses. For example, by adding a layer of absorptive material made of a material of known properties, one can control the thickness of the layer and reach the required value of the loss factor to achieve the amount of losses that satisfies the critical coupling condition. In these systems, when only a single resonant element is considered, it is possible to achieve a maximum sound absorption up to $\alpha=0.5$.

In the problem in reflection, the case of a single HR has been studied. In this case, it is possible to control the viscothermal losses in the system by optimizing the geometry of the HR. In this case, the geometrical parameters in the neck and the cavity can be tuned and allow the balance between the losses and 
leakage in the system and, for instance, the critical coupling condition. For the cases in reflection perfect sound absorption $(\alpha=1)$ can be achieved. 

Chapter 4

\section{Experimental evidence of critical coupling condition for perfect absorption for a single frequency with Helmholtz resonators}

In Chapter 3, the fundamentals of the TMM and the critical coupling condition are explained for two different configurations: a case in transmission and a case in reflection. These tools can be used to design a perfect absorbers.

The concepts detailed in the previous chapter are applied here in order to show an experimental evidence of critical coupling condition for perfect absorption. A HR is used in order to obtain perfect absorption at a single frequency.

First of all, the HR is designed and optimized with an algorithm based on the TMM. A numerical model is implemented by using the Finite Element Method (FEM) in order to validate the results of the optimization and, as a final step, the HR is manufactured and measured in an impedance tube. Thus, the sound absorption coefficient of the system is obtained analytically, numerically and experimentally. 
frequency with Helmholtz resonators

\subsection{Design and optimization of perfect absorbers}

The critical coupling condition, given when the leackage and the losses of the system are balanced, allows the perfect absorption in a system in reflection. In the particular case in which this balance is produced, the energy is trapped inside the resonator, dissipated in heat energy, and maximal absorption can be obtained [55]. In order to fulfill this condition, we tune the geometry of the HRs in order to find this balance. Since only one resonant element (the HR) is considered in this system, the critical coupling condition can be fulfilled for a single frequency and, for instance, perfect sound absorption can be achieved at that frequency.

An optimization by using the TMM has been implemented in order to obtain the geometrical parameters of the HR that maximize the sound absorption at the desired frequency. This optimization process is necessary to balance the viscothermal losses in the system to the leakage. The geometrical parameters (see Figure 3.16) to be optimized are the length and height of the neck of the $\mathrm{HR}, L_{n}$ and $h_{n}$, the length and height of the cavity of the HR, $L_{c}$ and $h_{c}$, and the length of the air cavity placed behind the resonator, $L$. The length and height of the slit formed between the upper part of the HR and the upper wall of the tube, $L_{s}$ and $h_{s}$ are also considered.

The optimization has been carried out by means of the Sequential Quadratic Programming (SQP) method, which is used for finding numerical solutions of constrained nonlinear optimization problems. The application of the SQP methodology to nonlinear optimization problems has the form [107]

$$
\begin{array}{ll}
\underset{x \in \Re^{n}}{\operatorname{minimize}} & f(x), \\
\text { subject to } & l \leq\left\{\begin{array}{c}
x \\
A x \\
c(x)
\end{array}\right\} \leq u,
\end{array}
$$

where $f(x)$ is a linear or nonlinear objective function, $c(x)$ is a vector of $m$ nonlinear constraint functions $c_{i}(x), A$ is a matrix, and $l$ and $u$ are vectors of lower and upper bounds.

The objective function selected for the optimization algorithm is the one that minimizes the reflection coefficient in a given frequency by tuning the geometrical parameters. This objective function can be mathematically defined as 
$|R(f)|^{2}=0$. For instance, based on Eq. (3.47), if the reflection is close to zero then perfect sound absorption $\left(\alpha_{\max }=1\right)$ will be obtained.

The resulting resonator, obtained after the optimization process and presenting perfect sound absorption at $f=250 \mathrm{~Hz}$, has the following geometrical characteristics: $L_{n}=10^{-3} \mathrm{~m}, h_{n}=10^{-2} \mathrm{~m}, L_{c}=0.0501 \mathrm{~m}$ and $h_{c}=0.0636$ $\mathrm{m}$. As it was mentioned before, no cavity is considered behind the resonator, so the parameter $L$ is equal to zero. This has been done in order to analyze the simplest case of study as the first step and check the validity of the models.

\subsection{Numerical model}

In order to confirm the result given by the optimization performed by using the TMM, a numerical simulation using the FEM has been implemented by considering the same configuration obtained in the previous step. The numerical simulations in FEM have been carried out using the commercial software COMSOL Multiphysics 5.3. Two modules of this software have been used: Acoustics and Acoustic-Structure Interaction.

The model that has been implemented (Figure 4.1) is a model that solves the Helmholtz equation in the frequency domain for a given frequency. Acoustic waves are propagated in a fluid (air) with harmonic variations of the pressure field. This harmonic variation is given by $e^{i \omega t}$.

The total acoustic pressure field is defined by the sum of the background pressure field of the sound wave propagating in the impedance tube (the one emitted by the sound source), $p_{b}$, and the scattered pressure field of the wave that is reflected by the sample (resonator), $p_{s}$.

$$
p_{t}=p_{b}+p_{s}
$$

where, $p_{b}=p_{0} e^{-i k x}$ and $p_{0}$ the amplitude of the incident wave.

Both the walls of the tube and the contours of the HR are considered as acoustically rigid, meaning that the normal component of the particle velocity is zero. 


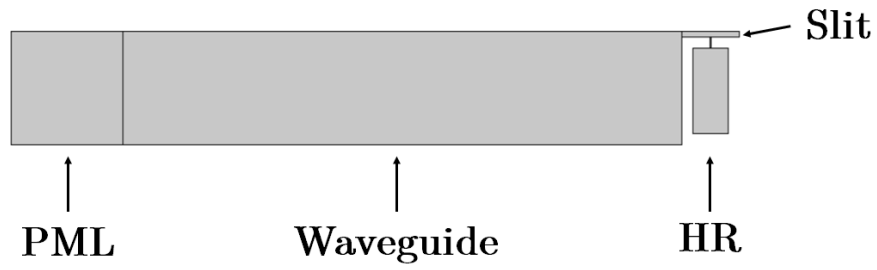

Figure 4.1: 2-D numerical model of the system.

For the domains including the cavity and neck of the HR and the slit formed on top of it, the equations for the viscothermal losses are applied (Eq. (3.56) and Eq. (3.57)).

Finally, in order to avoid unwanted reflections from the left end of the domain corresponding to the waveguide, a perfectly matched layer (PML) has been implemented to mimic an open and nonreflecting infinite domain.

\subsection{Experimental framework}

In the previous sections, the method to optimize the system of HRs has been shown, as well as the analytical and numerical models. Due to the applied nature of the work developed in this Thesis, and in order to validate the models previously applied, an experimental characterization is necessary. To do so, the designed HR has been manufactured in polylactic acid (PLA) by using a 3D printer Anycubic i3 Mega [108] and measured in an impedance tube. The experimental characterization of the system in terms of sound absorption has been carried out by means of the Transfer Function Method (TFM), specified in the Standard UNE-EN ISO 10534-2 [109].

This method consists of the generation of a signal containing energy in all the frequency range (for example, white noise), that is emitted by a sound source (loudspeaker), and measuring the pressure field in two points of the impedance tube by using two microphones placed between the sound source and the sample (in this case, the HR). With this information, it is possible to decompose the sound wave in its incident and reflected components and, for instance, obtaining the sound absorption coefficient. More details about this method can be found in Ref. [109]. 


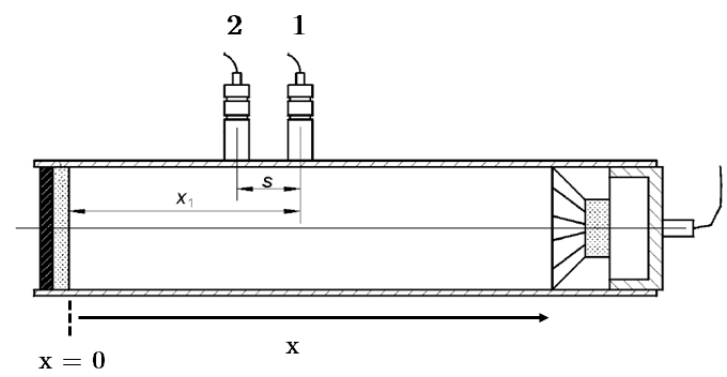

Figure 4.2: Scheme of the experimental set-up used for the Transfer Function Method (figure extracted from Ref. [109]).

Considering that only plane waves travel along the tube, the acoustic pressures at the position of both microphones, 1 and 2, can be written as

$$
\begin{aligned}
& p_{1}=\left|p_{I}\right| e^{i k_{0} x_{1}}+\left|p_{R}\right| e^{-i k_{0} x_{1}}, \\
& p_{2}=\left|p_{I}\right| e^{i k_{0} x_{2}}+\left|p_{R}\right| e^{-i k_{0} x_{2}},
\end{aligned}
$$

where, $\left|p_{I}\right|$ and $\left|p_{R}\right|$ are, respectively, the modules of the pressures of the incident and the reflected waves at the surface of the sample, $k_{0}$ is the wave number and $x_{1}$ and $x_{2}$ are the distances of the microphone 1 and 2 to the sample (note that the microphone 2 is the one closer to the sample).

The transfer function of the incident wave, $H_{I}$, is

$$
H_{I}=\frac{p_{2 I}}{p_{1 I}}=e^{-i k_{0}\left(x_{1}-x_{2}\right)}=e^{-i k_{0} s},
$$

being $s=x_{1}-x_{2}$ the distance between both microphones.

In the same way, it is possible to obtain the transfer function of the reflected wave, $H_{R}$, as

$$
H_{R}=\frac{p_{2 R}}{p_{1 R}}=e^{i k_{0}\left(x_{1}-x_{2}\right)}=e^{i k_{0} s} .
$$


The transfer function between the two microphones, $H_{12}$, for the total acoustic field can be obtained from Eqs. (4.4) and (4.5), keeping in mind that $\left|p_{R}\right|=$ $R\left|p_{I}\right|$ :

$$
H_{12}=\frac{p_{2}}{p_{1}}=\frac{e^{i k_{0} x_{2}}+R e^{-i k_{0} x_{2}}}{e^{i k_{0} x_{1}}+R e^{-i k_{0} x_{1}}} .
$$

From Eq. (4.6), the reflection coefficient can be experimentally obtained as

$$
R=\frac{H_{12}-H_{I}}{H_{R}-H_{12}} e^{2 i k_{0} x_{1}}
$$

The sound source used to emit the signal is a loudspeaker Fonestar UT-404 (see Figure 4.3b), with response from 60 to $12000 \mathrm{~Hz}$, sensitivity of $89 \mathrm{~dB}$ referred to $1 \mathrm{~W} / 1 \mathrm{~m}$ and a maximum acoustic pressure of $107 \mathrm{~dB}$ at $1 \mathrm{~m}$ of distance. Two Brüel \& Kjær 4189-A-021-1/2-inch free-field microphones with Type 2671 preamplifiers (see Figure 4.3c)) have been used to measure the acoustic pressure. These microphones have a frequency response from 20 to $20000 \mathrm{~Hz}$, and have been calibrated with a sensitivity of $47.2 \mathrm{mV} / \mathrm{Pa}$ and 54.3 $\mathrm{mV} / \mathrm{Pa}$, respectively, for a pure tone at a frequency of $1 \mathrm{kHz}$ with a sound pressure level of $94 \mathrm{~dB}$. 

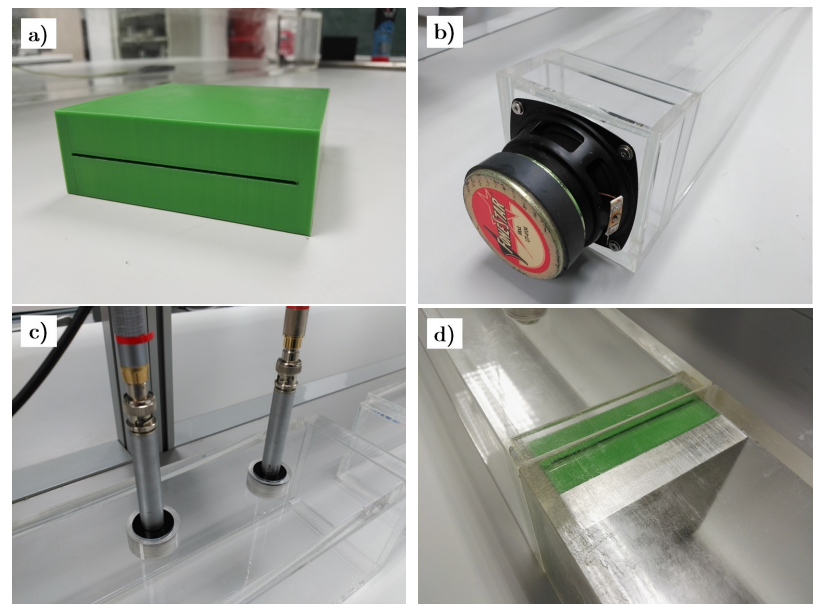

Figure 4.3: Experimental set-up used to measure the sound absorption coefficient of the HR: a) Helmholtz resonator manufactured in PLA, b) Sound source of the impedance tube, c) Microphones used to measure the pressure field in the tube, d) HR placed in the tube in contact with the rigid backing.

\subsection{Results}

The sound absorption coefficient has been theoretically, numerically and experimentally obtained by using the TMM, the FEM and the TFM, respectively. In Figure 4.4 the sound absorption coefficient obtained by means of the three methods is represented. 

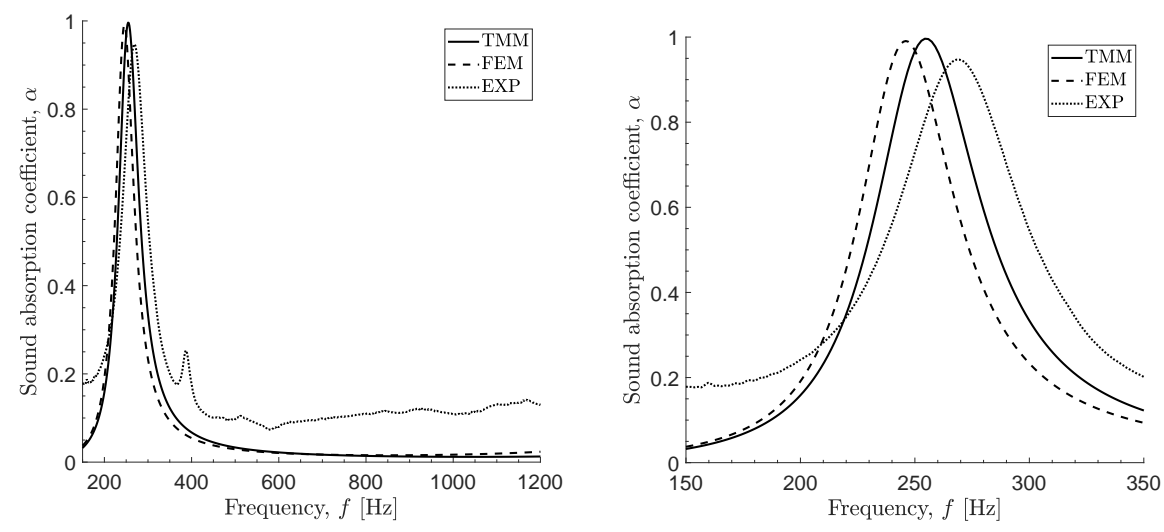

Figure 4.4: Left) Sound absorption coefficient of a single resonator optimized for $f=250$ $\mathrm{Hz}$, obtained with the TMM (solid line), FEM (dashed line) and experimentally (dotted line); Right) Zoom of the sound absorption coefficient at the resonance frequency.

A maximum of sound absorption coefficient, associated to the resonance of the HR, can be observed in Figure 4.4. This peak of absorption occurs in TMM at $254 \mathrm{~Hz}$, in FEM at $246 \mathrm{~Hz}$ and experimentally at $268 \mathrm{~Hz}$. This result proves that, by tuning the viscothermal losses of the system, it is possible to fulfill the critical coupling conditions and, for instance, obtaining perfect sound absorption. The difference between the resonance frequency obtained in TMM and the experimental measurement is $5.2 \%$, while the difference between the one obtained in FEM and experimentally is $5.6 \%$. This shows a good agreement between the three methods. The small disagreement between these three results may be given by different reasons: the limitations of the models with respect to the description of the experimental results, imperfections in the manufacturing process of the HR given by the precision of the 3D printer, or the presence of additional losses associated to the joints of the impedance tube.

Due to the fact that a single resonant structure is being used and that no air cavity is considered between the HR and the rigid backing, only one peak of absorption appears. If we keep in mind the original problem that is being faced in this Thesis, which is the mitigation of noise during the lift-off of spacecrafts, we need to implement a solution that broadens this peak. In order to obtain a peak of absorption broader in frequency, it is necessary to include more resonant elements in the system separated by air cavities. These new 
HRs need some specific geometrical characteristics that enable the coupling between the modes of the system and the balance of the viscothermal losses in order to achieve broadband sound absorption in a designed frequency range. The implementation of this solution is detailed in Chapter 5 .

\subsubsection{Complex frequency plane}

The reflection coefficient can be also represented in the complex frequency plane, in which the zeros (blue) and poles (red) of the system can be observed. In a problem in reflection as the one considered in this work, $R$ represents the scattering of the system and the zeros of the system correspond to the cases in which the incident wave is totally absorbed $[53,55]$. The reflection coefficient has pairs of poles and zeros that are complex conjugate, where the poles have a positive imaginary part and the zeros have a negative imaginary part [55].

The complex frequency plane is obtained by considering a complex frequency defined as $\omega=\omega_{r}+i \omega_{i}$, being $\omega_{r}$ and $\omega_{i}$ the real and imaginary parts of the frequency. The position of the zeros and poles of the scattering matrix of the system in the complex frequency plane characterizes the physical reflection properties along the real frequency axis (note that the sign of the imaginary part of the zeros and poles of the system depends on the time convention). This is due to the fact that their position is associated to the eigenvalues of the system and, as it has been previously detailed, the condition for the critical coupling is fulfilled when the eigenvalues are equal to zero. In this case, the zeros of the reflection coefficient cross the real frequency axis.

Figure 4.5 shows the representation of $\log |R|^{2}$ as a function of the real and imaginary part of the frequency. In the lossless case each pole presents a zero that is its complex conjugate [54] associated to the leakage of the HR. In this case, as it is shown in Figure 4.5a), the zeros and poles of the reflection coefficient are placed equidistant to the real frequency axis. In the lossy case (Figure 4.5b)) the impedance matching is fulfilled when the zero of the reflection coefficient crosses the real axis. This happens when the loss factor added to the system is exactly the leakage of the HR [55]. The zeros of the reflection coefficient in the complex frequency plane lay on the real axis, meaning that the critical coupling condition has been fulfilled and, as a consequence, perfect absorption is achieved. 

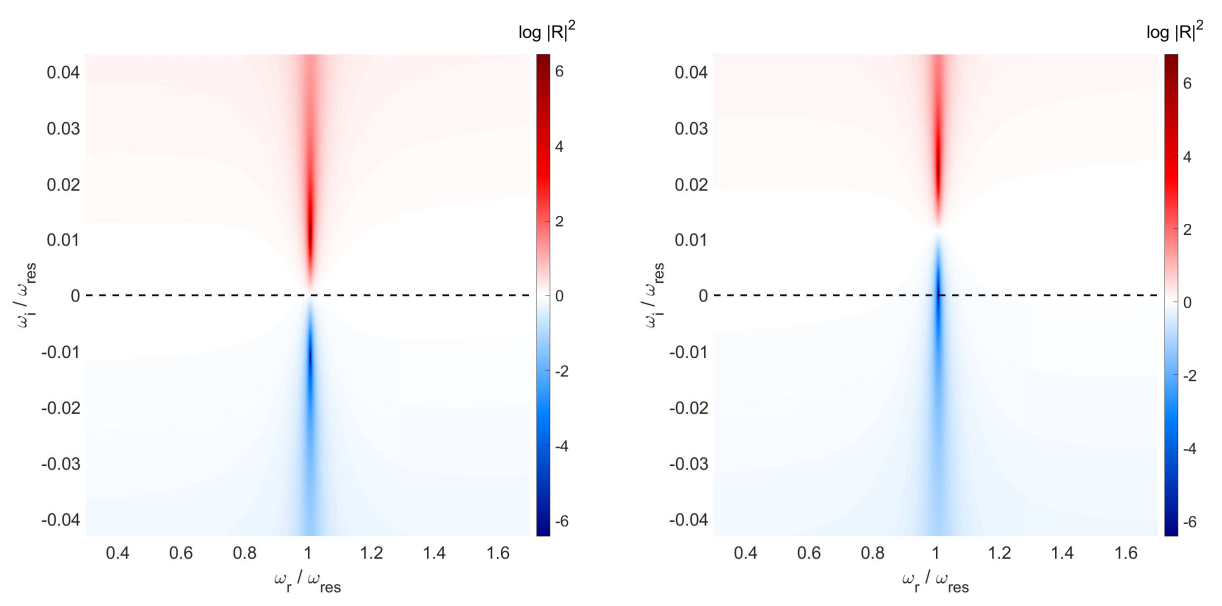

Figure 4.5: Representation of $\log |R|^{2}$ in the complex frequency plane of a system composed by a single $\mathrm{HR}$ with resonance frequency of $250 \mathrm{~Hz}$ : a) Lossless case, b) Lossy case fulfilling the critical coupling condition.

\subsection{Conclusions}

In this chapter, we have presented the experimental evidence of the critical coupling condition for perfect sound absorption for a single frequency by using a HR. This has been done analytically, numerically and experimentally.

During this chapter, and in the upcoming ones, the work is focused in the problem in reflection. This is given by the nature of the problem that we are facing in this Thesis, in which the launch pad acts as a rigid backing and prevents sound transmission.

Perfect sound absorption has been achieved for a single frequency because one resonating element has been used. Nevertheless, since the acoustic spectrum generated during the ignition and first stage of the lift-off of the spacecraft is broadband, our interest is increasing the range of frequencies for the perfect sound absorption. To do so, the methodology used along this chapter will be used in Chapter 5 in order to design a system composed by a larger number of HRs. 
Chapter 5

\section{Design of a finite distribution of Helmholtz resonators for broadband absorption}

In Chapter 3, the requirements for achieving critical coupling condition were studied in two different configurations: the problem in transmission and the problem in reflection. The original problem that we want to face in this Thesis is the mitigation of the high sound pressure level existing at the launch pad during the ignition and first stages of the lift-off of the spacecraft. Thus, we are facing a problem in reflection. This is due to the fact that the bottom of the launch pad acts as a rigid backing, reflecting the acoustic waves back to the rocket and, as a consequence, no transmission of the waves is allowed beyond it.

Open systems, such as HRs, are characterized at the resonance frequency by both the leakage of energy (i.e., the coupling of the resonant elements with the propagating medium) and the intrinsic losses of the resonator [58]. When these two phenomena are balanced, condition known as critical coupling, perfect sound absorption can be achieved in the system by trapping the energy around the resonant elements [57].

In the case of purely reflecting systems, either symmetric and anti-symmetric resonances that are critically coupled can be used to obtain perfect absorption 
of energy [58], even if only a single resonating element is considered. To do so, the system must be tuned in order to find the appropriate amount of losses to fulfill the critical coupling condition. Nevertheless, as it was demonstrated in Chapter 3, in this case perfect absorption occurs only at one frequency.

The bandwidth of the peak of absorption can be increased by adding more resonant elements to the system in a way that they are critically coupled. The achievement of very efficient low-frequency broadband absorption is the result of a fine tuning of the attenuation properties with resonance characteristics of the structure that leads to the critical coupling of the resonant modes [54, 55]. By tuning the geometry of the resonating elements of the system, the reflection coefficient can be tailored to obtain either a custom reflection phase, moderate or even perfect absorption [54]. This method was used by Jiménez et al. [54] to design a set of 9 monopolar resonators with graded dimensions to achieve quasi-perfect sound absorption from 300 to $1000 \mathrm{~Hz}$ in the problem in transmission.

In this chapter, we consider a system formed by an array of HRs separated by air cavities and discuss the condition for broadband absorption. The hypothesis is that these cavities introduce additional modes in the system given by the interaction of each cavity with the adjacent HRs. These modes would increase the performance of the system regarding to noise mitigation by means of the absorption and diffusion of sound waves, as will be studied in Chapter 6 . The system is placed into an impedance tube, and a slit is formed between the top part of the resonators and the upper wall of the tube. Furthermore, the consideration of these cavities behind the HRs add degrees of freedom to the design of the system. The tuning of the losses of the system is done by means of the optimization algorithm implemented using the TMM as shown in Chapter 3 .

The Chapter is structured as follows: in Section 5.1 we apply the analytical methods that were used in Chapter 3 to tune the geometry of each HR in a multilayer system, and present the method to obtain the Insertion Loss in Reflection, $I L R$, in Section 5.2. The resulting geometry is presented in the Section 5.3 and used in Section 5.4 to implement a numerical model to characterize the sound absorption and $I L R$ by using the FEM. In Section 5.5, the experimental methodology and set-up used to measure the sound absorption coefficient is detailed. Finally, the results of all the parameters calculated for the system are introduced and discussed in Section 5.6. 


\subsection{The Transfer Matrix Method for a multilayer system}

The fundamentals of the TMM to study the conditions for critical coupling in a system composed by a single Helmholtz resonator were detailed in Chapter 3. This method relates the pressure and particle velocity at both sides of a system:

$$
\left[\begin{array}{l}
p \\
v
\end{array}\right]_{x=0}=\mathbf{M}\left[\begin{array}{l}
p \\
v
\end{array}\right]_{x=L}=\left[\begin{array}{ll}
M_{11} & M_{12} \\
M_{21} & M_{22}
\end{array}\right]\left[\begin{array}{l}
p \\
v
\end{array}\right]_{x=L}
$$

The TMM is used when the whole system can be represented into a sequence of subsystems that interact only with adjacent systems [76]. Thus, the transmission matrix of a multilayer system of resonating elements can be obtained by calculating the product of the matrix of each individual element:

$$
M=\prod_{n=1}^{N} M_{\Delta_{s l i t}}^{[n]} M_{s}^{[n]} M_{H R}^{[n]} M_{s}^{[n]} M_{\Delta_{s l i t}}^{[n]} M_{w}^{[n]}
$$

where $M_{\Delta l_{s l i t}}^{[n]}$ is the radiation correction of the slit to the free space, $M_{s}^{[n]}$ in the transmission matrix for each slit, $M_{H R}^{[n]}$ is the transmission matrix for each resonator and $M_{w}^{[n]}$ is the transmission matrix for the waveguide. The index $n$ indicates the position of the HR in the system. The definition of these parameters was given in Chapter 3.

\subsection{Insertion Loss in Reflection}

The Insertion Loss $(I L)$ is a parameter used to characterize linear systems in many fields. This parameter is defined as the difference in sound pressure levels measured before and after a barrier or silencer installed in the line between the source and the receiver [75]. This magnitude represents the attenuation provided by the insertion of a structure between the noise source and the receiver, and it is an estimation of reduction of the transmitted acoustic energy.

In the problem of noise mitigation during the rocket's lift-off, both the noise source and the receiver are placed at the same side. For this reason, a new definition of the concept of $I L$ is required. In Ref. [49], the authors adapt the original definition of $I L$ to obtain this magnitude for systems in reflection (Insertion Loss in Reflection, ILR). The definition of the ILR is based on 
the calculation of the difference (in $\mathrm{dB}$ ) between the total sound pressure level measured in a reference situation, $P_{\text {ref }}$, with respect to the total sound pressure level measured when the system of HRs has been placed at the same position, $P_{H R}$. The structure that is used as a reference in this study is a flat rigid backing. The $I L R$ can be expressed, in $\mathrm{dB}$, as follows:

$$
I L R \equiv L_{r e f}-L_{H R}=10 \log _{10}\left(\frac{\left|P_{r e f}\right|^{2}}{\left|P_{H R}\right|^{2}}\right)
$$

where, $L_{r e f}$ and $L_{H R}$ the sound pressure level in both cases, $P_{r e f}$ and $P_{H R}$ are the pressures measured in the case of reference and placing the system of HRs, respectively.

The ILR of the system proposed in this chapter has been experimentally obtained from the measurements carried out in the impedance tube. By developing Eq. (5.3), and keeping in mind that the reflection coefficient can be expressed as the ratio between the pressure of the reflected wave and that of the incident wave $\left(R=P_{r} / P_{i}\right)$, we can express the $I L R$, in $\mathrm{dB}$, as follows:

$$
I L R=10 \log _{10}\left(\frac{1}{\left|R_{H R}\right|^{2}}\right)
$$

An interesting tool for the study of the critical coupling condition and, for instance, to interpret the phenomenon of perfect absorption is the complex frequency plane. As it was mentioned in Chapter 3, in a problem in reflection as the one considered in this work, $R$ represents the scattering of the system and the zeros of the system correspond to the cases in which the incident wave is totally absorbed [110].

\subsection{Design and optimization of the system}

A system composed by a distribution of five Helmholtz resonators has been designed for achieving broadband perfect sound absorption. The goal is to obtain the geometrical parameters of the resonators that maximize the sound absorption in a range of frequencies from $400 \mathrm{~Hz}$ to $560 \mathrm{~Hz}$ (a bandwidth of $160 \mathrm{~Hz}$ ). The frequency range has been selected based on the VEGA C User's Manual [111], in which the acoustic noise spectrum under the fairing in flight is shown (see Table 5.1). 
Table 5.1: Flight limit level $[\mathrm{dB}]$ in the acoustic noise spectrum under the fairing in flight (extracted from [111]).

\begin{tabular}{|c|c|c|}
\hline $\begin{array}{c}\text { Octave center } \\
\text { frequency }[\mathbf{H z}]\end{array}$ & $\begin{array}{c}\text { Lift-off } \\
(\mathbf{t}=[\mathbf{0}-\mathbf{3}] \mathbf{s})\end{array}$ & $\begin{array}{c}\text { Atmospheric phase } \\
(\mathbf{t}>\mathbf{3} \mathbf{~ s})\end{array}$ \\
\hline 31.5 & 112 & 110 \\
\hline 63 & 123 & 120 \\
\hline 125 & 126 & 122 \\
\hline 250 & 136 & 127 \\
\hline 500 & 139 & 130 \\
\hline 1000 & 127 & 127 \\
\hline 2000 & 122 & 118 \\
\hline$O A S P L(20-2828 \mathrm{~Hz})$ & 141 & 133.7 \\
\hline
\end{tabular}

Both the acoustic noise spectrum during the lift-off and the atmospheric phase shown in Table 5.1 can be represented graphically integrated in octave frequency bands (Figure 5.1). In this Thesis the work is focused in the liftoff phase, comprising the first 3 seconds of the launching process. It can be observed that the octave frequency bands in which more acoustic energy is released are the one whose central frequencies are $250 \mathrm{~Hz}$ and $500 \mathrm{~Hz}$.

In Chapter 3 it was analytically, numerically and experimentally demonstrated that perfect absorption can be achieved at a single frequency when one resonant element is tuned properly in the system by modifying its geometrical properties to match the viscothermal losses. Nevertheless, given that the frequency range in which the $S P L$ is broader, it is necessary to increase the range of perfect absorption. Thus, the proposed solution of a system composed by five HRs has been oriented to the absorption of the sound pressure field in frequencies within this range. The consideration of five HRs increases the bandwidth of sound absorption in the range of target frequencies that can be extracted from the spectrum presented in Figure 5.1.

The geometry of the HRs has been tuned by using the optimization algorithm based on the TMM that was presented in Chapter 3. The geometrical parameters of every HRs to be optimized (see Figure 5.2) are the length and height of the neck of the HR, $L_{n}$ and $h_{n}$, the length and height of the cavity of the $\mathrm{HR}, L_{c}$ and $h_{c}$, and the length of the air cavity placed behind the resonator, $L$. The length and height of the slit formed between the upper part of the HR and the upper wall of the tube, $L_{s}$ and $h_{s}$ are also considered. In this case, the optimization has been carried out considering the five HRs as a single system. Thus, the geometry of the five HRs have been optimized simultaneously 


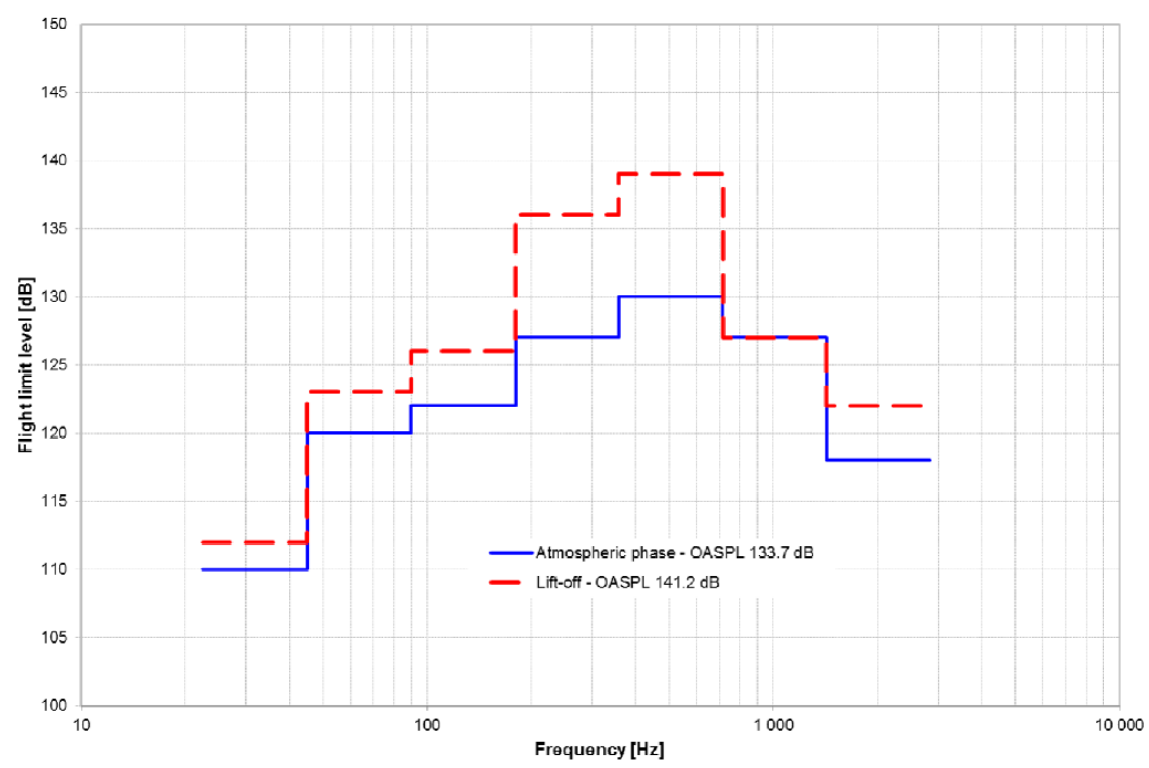

Figure 5.1: Acoustic noise spectrum under the fairing in flight (extracted from [111]).

in order to consider the interactions that will occur between them when the complete system is being measured.

In this system, the reduction in the reflection coefficient is produced via the increase of the losses in the system. The optimization algorithm has the tendency, to reduce some dimensions to values of the geometrical parameters as small as possible as a way to maximize the viscothermal losses. This represents an inconvenient from a constructive point of view if some dimensions are in a range lower than $1 \mathrm{~mm}$, given that the size of the nozzle of the 3 -D printer that will be used is $0.4 \mathrm{~mm}$ (more details are given in Section 5.5). Thus, we introduce lower bounds for the values of some of them. Keeping this in mind, we have set the lower bounds of $L_{n}=1 \mathrm{~mm}$ and $L_{c}=10 \mathrm{~mm}$. The thickness of the walls of the HRs has been chosen to be fixed to $t=10 \mathrm{~mm}$ with the purpose to avoid the problem of the excitation of the walls of the HR by the interaction of the sound wave with the structure, which may cause that they radiate sound backwards, and also to ensure the proper manufacturing of the system. The geometrical parameters obtained for each resonator after the optimization process can be seen in detail in Table 5.2. The system has a total length of $31.1 \mathrm{~cm}$ and a height of $10.1 \mathrm{~cm}$ (corresponding to the height of the impedance tube). It is important to note that the HRs must be placed 


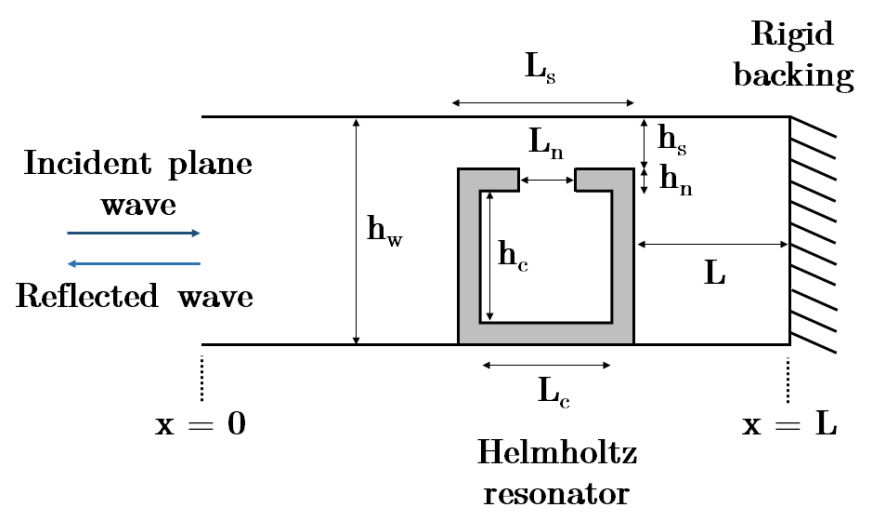

Figure 5.2: Scheme of a system composed by a single resonator and the geometrical parameters to be optimized.

as they are numbered in Table 5.2, since the modification in the order would modify the geometry of the system and the conditions for achieving the critical coupling would not be fulfilled.

Table 5.2: Optimized geometrical parameters obtained for each Helmholtz resonator composing the system.

\begin{tabular}{|c|c|c|c|c|c|}
\hline Parameter [mm] & HR1 & HR2 & HR3 & HR4 & HR5 \\
\hline$h_{n}$ & 5.20 & 32.39 & 38.61 & 24.78 & 10.46 \\
\hline$L_{n}$ & 4.63 & 1.00 & 1.00 & 1.00 & 1.67 \\
\hline$h_{c}$ & 49.77 & 34.92 & 42.06 & 47.13 & 75.52 \\
\hline$L_{c}$ & 46.34 & 10.00 & 10.00 & 10.00 & 16.74 \\
\hline$h_{s}$ & 36.03 & 23.69 & 10.34 & 19.09 & 5.02 \\
\hline$L_{s}$ & 66.35 & 30.00 & 30.00 & 30.00 & 36.74 \\
\hline$L$ & 25.00 & 25.00 & 25.00 & 23.00 & 20.00 \\
\hline
\end{tabular}

The first resonance frequencies of each resonators resulting after the optimization process can be obtained from the frequency that fulfill the condition $\Im(Z)=0$, in which all the correction lengths are considered. In this configuration, the first resonance frequencies of the five HRs are, respectively, $f_{H R 1}=1074 \mathrm{~Hz}, f_{H R 2}=514 \mathrm{~Hz}, f_{H R 3}=429 \mathrm{~Hz}, f_{H R 4}=505 \mathrm{~Hz}$ and $f_{H R 5}=615 \mathrm{~Hz}$. Given these values, it can be observed that $f_{H R 1}$ and $f_{H R 5}$ are out of the range of frequencies of the optimization (400 to $560 \mathrm{~Hz}$ ). In this case, the array of the HRs and the air cavities placed between them excite coupled modes in the system that result in new properties in the system, such 


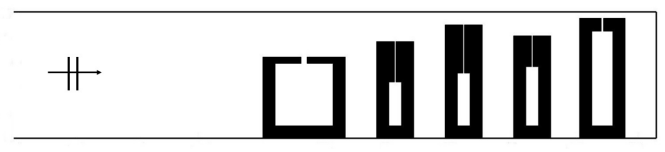

Figure 5.3: Tuned geometry of the optimized system for perfect sound absorption from 400 to $560 \mathrm{~Hz}$.

as diffusion of sound waves. Since the study that is carried out in this chapter is done by using an impedance tube, it's not possible to analyze these new properties due to the characteristics of the set-up. The diffusive properties of the system associated to the cavities will be presented in Chapter 6. A 2 -D view of the resulting system is represented in Figure 5.3 and the technical drawings can be seen in the Appendix (page 135).

\subsection{Numerical analysis methods}

Numerical simulation methods have evolved significantly over the last years, which has gifted these new technologies plenty of increasingly important advantages, such as an easy visualization of some phenomena and the possibility of having an intermediate step between the theoretical and the experimental phase of the work [112].

The Finite Element Method (FEM) has been conceptually developed for the numerical discretization of problems on bounded domains [113]. In the FEM, the solution region is considered as built up of many small, interconnected subregion called finite elements [114]. The application of this method is particularly interesting when hand calculations cannot provide accurate results [115], the model to be developed has a complex geometry or the range of parameters to be studied is very wide.

A numerical model has been implemented by means of the FEM following the same procedure detailed in Chapter 3. For this reason we urge the reader to consult this Chapter for more details. This numerical model is composed by 88812 domain elements and 4708 boundary elements, and has been solved for a total of 182104 degrees of freedom. The resulting geometry of the model is shown in Figure 5.4.

The sound absorption coefficient of the system, $\alpha$, has been obtained by setting two measurement points placed at a certain distance of the system. The total 


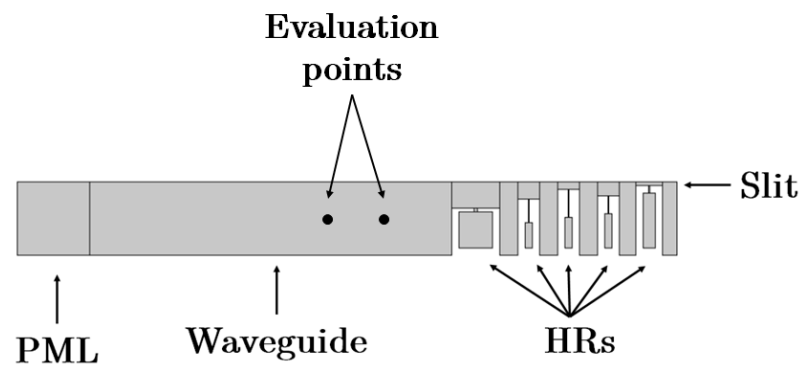

Figure 5.4: Scheme of the model implemented to be solved by using FEM.
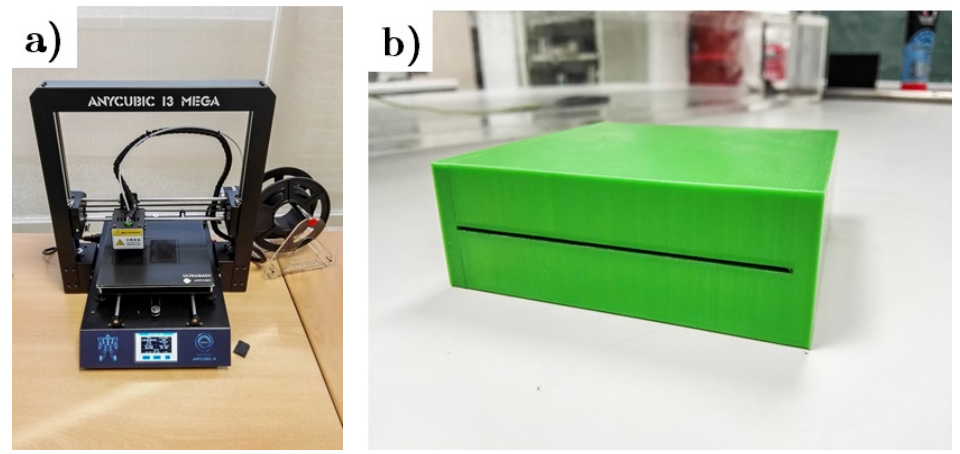

Figure 5.5: a) 3D printer used to manufacture the HRs, b) One of the structures that has been manufactured.

sound pressure field has been evaluated in both points, and $\alpha$ has been obtained by means of the Transfer Function Method [109]. This method is explained in detail in the Section 4.3.

\subsection{Manufacturing and set-up}

The system resulting from the optimization process has been manufactured following the same procedure shown in Chapter 4. A picture of the 3-D printer that was used for this procedure is shown in Figure 5.5a), and the resulting HR can be observed in Figure 5.5b).

The complete set-up can be observed in Figure 5.6. In this picture we can observe the sound source, placed at the left side of the tube, that emits the signal generated by the sound card. The sound pressure, both of the incident 


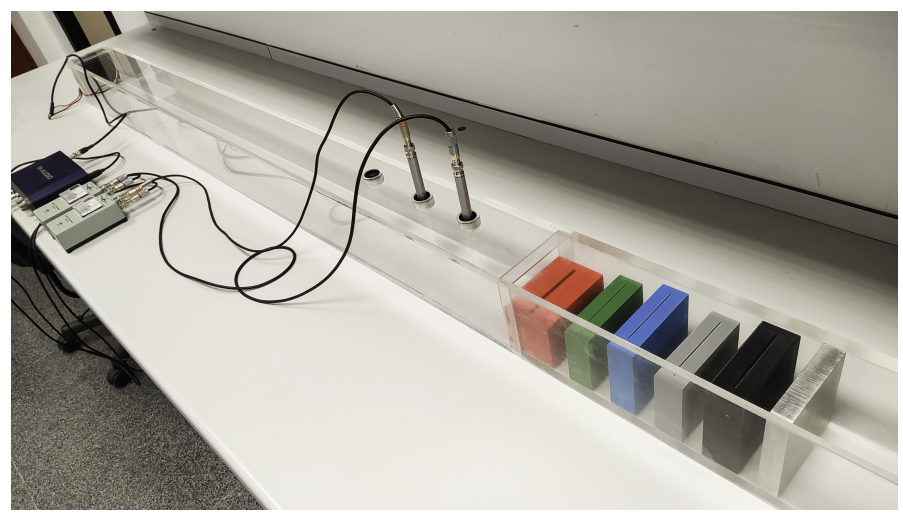

Figure 5.6: Experimental set-up used for the Transfer Function Method.

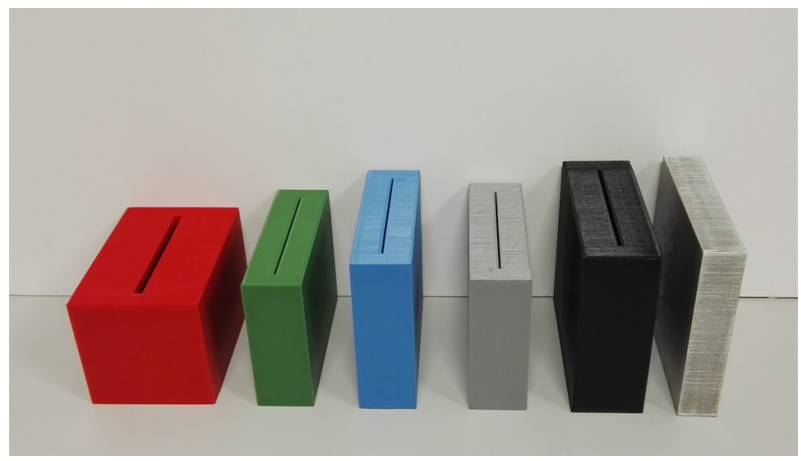

Figure 5.7: Distribution of the HRs in the system (outside of the tube).

and the reflected waves, is measured by the two microphones. The distribution of the HRs in the system (outside of the tube) is shown in Figure 5.7.

\subsection{Results and discussion}

\subsubsection{Study of the sound absorption}

A system composed by five Helmholtz resonators has been designed and optimized in order to present broadband high sound absorption in a range of frequencies from 400 to $560 \mathrm{~Hz}$. 


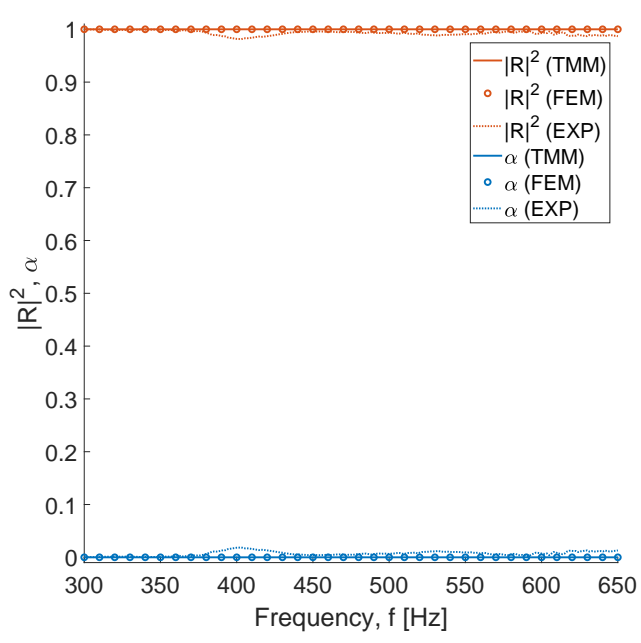

Figure 5.8: Sound reflection (red) and absorption (blue) coefficients of a rigid backing in TMM (solid line), FEM (dashed line) and experimental measurements (dotted line).

The used methodology consists in the design and optimization of the system by means of the TMM, the validation of this optimization by using FEM (full wave calculations) and, as a last step, checking the final result using the experimental measurements based on the TFM.

Following this methodology, the sound absorption coefficient of a flat rigid surface has been obtained to be used as a reference case, in order to check the proper functioning of the three methods. The flat rigid surface behaves as a perfect reflector and, as a consequence, absorption with a constant value with frequency of $\alpha=0$ is obtained. Figure 5.8 shows the absorption coefficient of the rigid surface with the three methods. This figure shows a good agreement between the sound absorption coefficient obtained both with the TMM and the FEM, presenting a perfect overlap in the axis $\alpha=0$.

For the case of the experimental measurement, it is possible to observe that $\alpha$ is slightly above zero at some frequencies. Since the impedance tube is composed of three different parts, the joints between them may introduce additional leakage that slightly increase $\alpha$.

The sound absorption coefficient of the system has been obtained by using TMM and experimental measurements. The results obtained using these meth- 


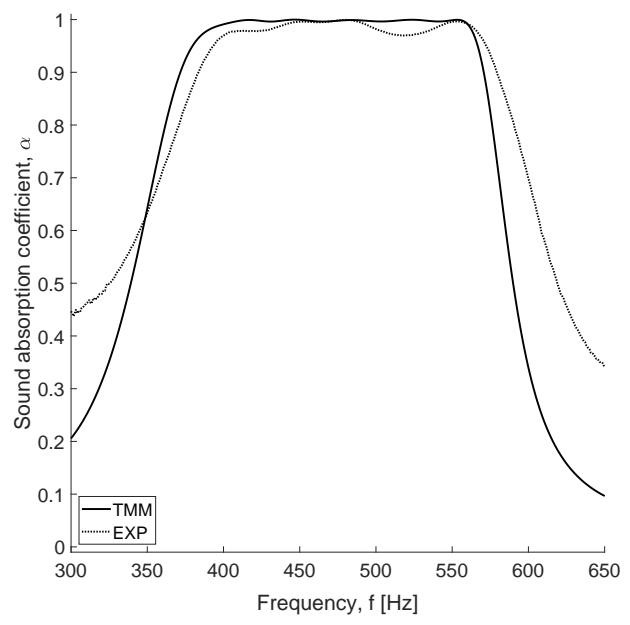

Figure 5.9: Sound absorption coefficient of the system of HRs in TMM (solid line) and experimental measurements (dotted line).

ods are shown in Figure 5.9. As it can be observed in this figure, a broad peak of absorption has been achieved in the designed range of frequencies.

This peak of absorption is mainly associated to the individual resonances of each HR and the effect of coupled modes between HRs and their adjacent cavities. The resonance frequencies of the resonators are close to each other and, as a consequence, the overlapping of the modes of the HRs is produced due to the critical coupling.

A good agreement is observed between the analytical predictions with TMM and the experimental measurements in the optimized range of frequencies. The disagreements that can be observed between both results may be caused by the imperfections of the experimental set-up, such as the joints of the parts of the impedance tube or the imperfect fitting of the HRs into the waveguide, or limitations in the analytical model.

The results for the sound absorption coefficient shown in Figure 5.9 are synthesized in Table 5.3 and Table 5.4 in order to show both the ranges of frequencies and the total bandwidth with sound absorption higher than a given value, respectively. 
Table 5.3: Ranges of frequencies with sound absorption higher than $0.9,0.95$ and 0.99 .

\begin{tabular}{|l|c|c|c|}
\hline & $\boldsymbol{\alpha}>\mathbf{0 . 9}$ & $\boldsymbol{\alpha}>\mathbf{0 . 9 5}$ & $\boldsymbol{\alpha}>\mathbf{0 . 9 9}$ \\
\hline TMM & $372-570 \mathrm{~Hz}$ & $380-566 \mathrm{~Hz}$ & $399-561 \mathrm{~Hz}$ \\
\hline EXP & $384-579 \mathrm{~Hz}$ & $393-572 \mathrm{~Hz}$ & $440-494 \mathrm{~Hz}$ \\
& & & $544-561 \mathrm{~Hz}$ \\
\hline
\end{tabular}

Table 5.4: Total bandwidth $(\Delta f)$ of the peaks of sound absorption higher than $0.9,0.95$ and 0.99 .

\begin{tabular}{|l|c|c|c|}
\hline & $\boldsymbol{\alpha}>\mathbf{0 . 9}$ & $\boldsymbol{\alpha}>\mathbf{0 . 9 5}$ & $\boldsymbol{\alpha}>\mathbf{0 . 9 9}$ \\
\hline TMM & $198 \mathrm{~Hz}$ & $186 \mathrm{~Hz}$ & $162 \mathrm{~Hz}$ \\
\hline EXP & $195 \mathrm{~Hz}$ & $179 \mathrm{~Hz}$ & $71 \mathrm{~Hz}$ \\
\hline
\end{tabular}

It follows from the results shown in Tables 5.3 and 5.4 that a sound absorption coefficient higher than $\alpha>0.9$ occurs in a range of frequencies of almost 200 $\mathrm{Hz}$ bandwidth in both TMM and experimentally. If the threshold of the sound absorption coefficient is increased to $\alpha>0.95$, one can observe that the range of frequencies is still broad, achieving a value of around $180 \mathrm{~Hz}$.

The sensitivity of the conditions for the critical coupling increase as $\alpha$ tends to unity. For this reason, for the case of $\alpha>0.99$, the agreement of the results varies. This case is the one in which the required conditions for the critical coupling play the most important role. The sources of disagreement have a more visible effect in this case, due to the fact that small changes in the system could break the required balance by modifying the total amount of losses in the system.

The representation in the complex frequency plane of the poles (red) and zeros (blue) of the reflection coefficient as $\log |R|^{2}$ is shown in Figure 5.10. The zeros are located in the negative imaginary plane of the complex frequency plane due to the $e^{i \omega t}$ sign convention. In the optimized range of frequencies, the zeros cross the real frequency axis and in the vicinity, meaning that the energy is trapped around the HRs and, as a consequence, the critical coupling condition is fulfilled. 


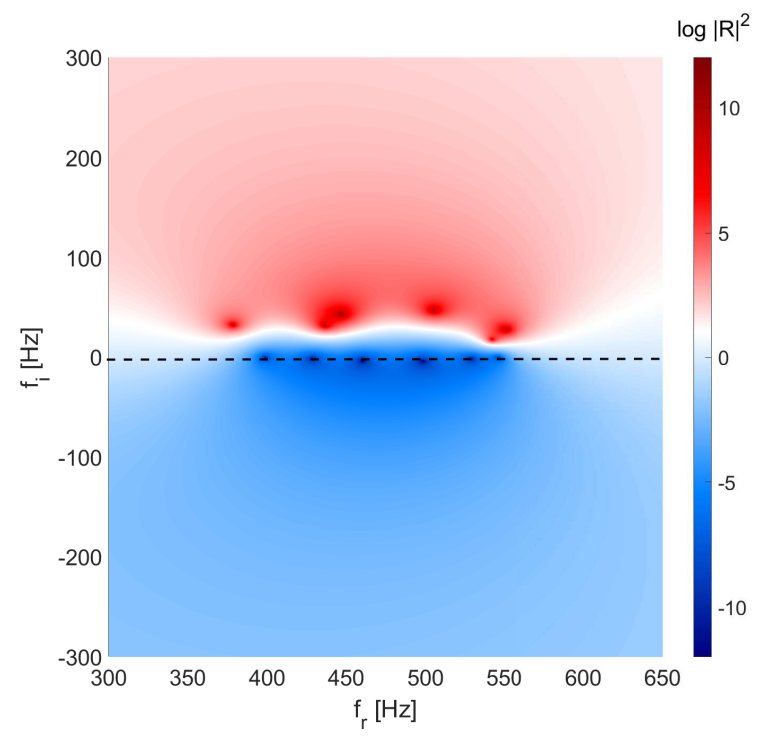

Figure 5.10: Complex frequency plane of the reflection coefficient calculated using the TMM, where $f_{r}$ and $f_{i}$ are, respectively, the real and imaginary parts of the complex frequency.

\subsubsection{Insertion Loss in Reflection}

The $I L R$ has been obtained experimentally. The resulting $I L R$ is shown in Figure 5.11 for the full range of working frequencies of the impedance tube (from 200 to $1700 \mathrm{~Hz}$ ). As it can be observed in the figure, a positive ILR has been obtained in the complete range, with a higher value in the band with central frequency of $500 \mathrm{~Hz}$. This band coincides with the frequency range for which the system has been optimized (from 400 to $560 \mathrm{~Hz}$ ). In this range, the value of the $I L R$ varies between 15.2 and $32.1 \mathrm{~dB}$. The average $I L R$ in this range is $20.1 \mathrm{~dB}$ (see Figure 5.12). 

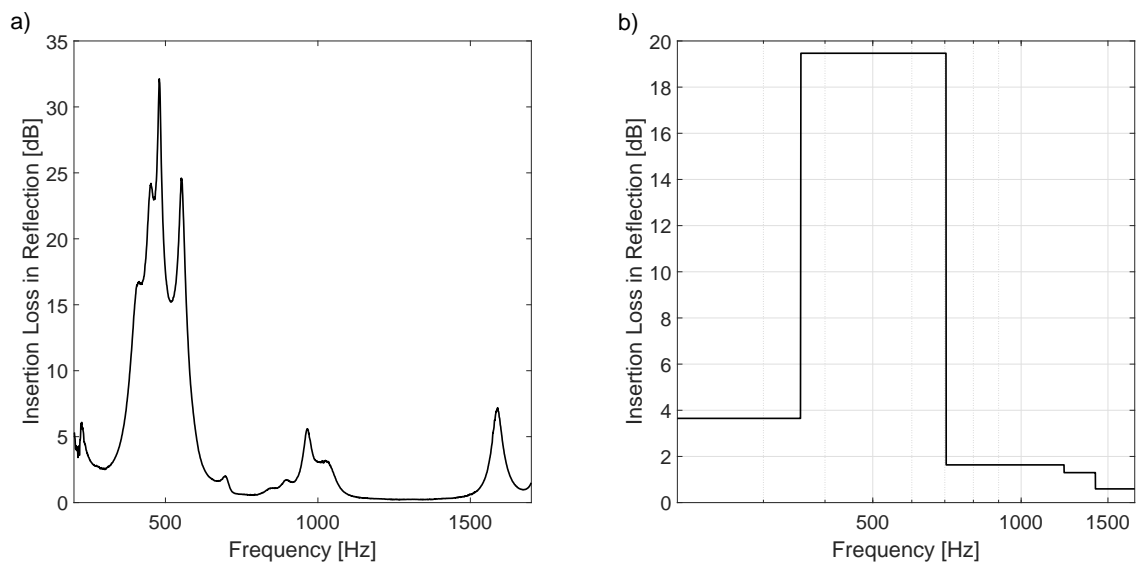

Figure 5.11: Insertion Loss in Reflection $(I L R)$ of the system in the full range of working frequencies of the impedance tube; a) with no integration; and b) integrated in octave frequency bands.

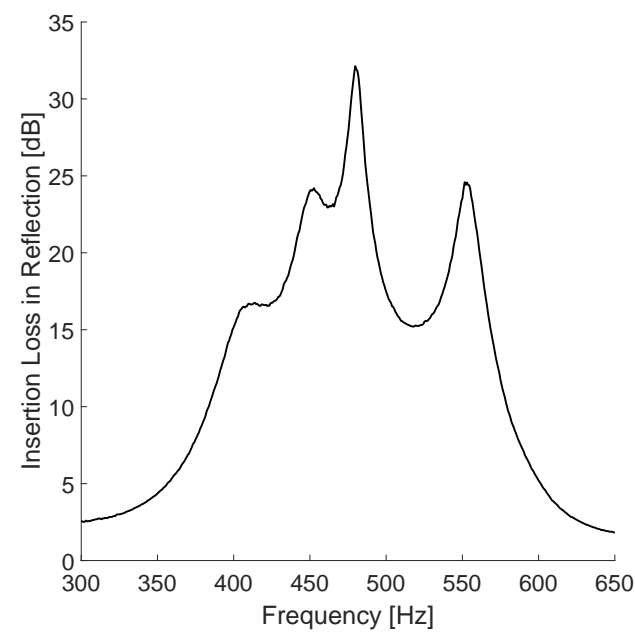

Figure 5.12: Insertion Loss in Reflection $(I L R)$ of the system in the optimized range of frequencies.

The high $I L R$ is associated to the broadband peak of sound absorption (and, for instance, low reflection) of the system in this range of frequencies. In other words, placing the proposed system of HRs results in a lower pressure field in 
front of the sample than in the case of a rigid flat surface due to the fact that it is absorbing most of the energy.

Figure 5.12 shows the ILR of the system in which four main peaks can be observed at $410 \mathrm{~Hz}, 450 \mathrm{~Hz}, 480 \mathrm{~Hz}$ and $550 \mathrm{~Hz}$. Comparing the result of this figure with the one shown in Figure 5.10, it is possible to find the concordance between them, since the zeros of the system cross the real frequency axis at these frequencies.

\subsection{Conclusions}

In this Chapter we have reported broadband perfect acoustic absorption in low frequency regime for a problem in reflection. A system composed by five critically coupled HRs separated by air cavities has been proposed in order to absorb the sound waves in the frequency range between 400 and $560 \mathrm{~Hz}$. We have shown that by using an optimization process it is possible to tune the geometry of the HRs and the cavities behind them to adapt the inherent losses of the HRs and maximize the sound absorption over a given frequency range.

We have made use of the analysis of the complex frequency plane (also known as zeros-poles diagram). This tool has proved to be very useful to identify the critical coupling condition. In this case, the system has been studied in an impedance tube, in which plane waves in stationary regime are propagated. This configuration allowed to characterize the behavior of the system when all the parameters are under control.

The proposed configuration is the first proof of concept of a system to be applied in the launch pad for the mitigation of the acoustic field. This work sets the base for the next step in our study, in which a bidimensional distribution of HRs will be studied in an anechoic chamber. The details of this work are given in the next Chapter. 
Chapter 6

\section{Design of a bi-dimensional distribution of Helmholtz resonators for broadband absorption}

In Chapter 5, the properties for sound absorption of a system composed of five HRs were studied. This system was designed by means of an optimization algorithm that tuned the geometrical parameters of each HR in order to achieve perfect sound absorption in a given frequency range (in this case, from 400 to $560 \mathrm{~Hz}$ ). In the proposed system, we considered plane waves propagating in one dimension.

In this Chapter a new goal has been set: studying the behavior of the system in terms of noise mitigation in free field conditions. A distribution composed of five rows of HRs, each one composed itself of five HRs separated by cavities is studied. The system is based on the same distribution that the one analyzed in Chapter 5. We notice here that the geometry of Chapter 5 implies rigid condition at the upper and lower walls of the tube, which is equivalent, in the optimized range of frequencies, to a periodic boundary condition. Therefore, the geometry analyzed in Chapter 5 represents a semi-infinite periodic wall radiated by a plane wave. In this chapter we want to study the effects of finite 
absorption

size in the system and how they can modify (or not) the desired effects of absorption.

In this case, the system is formed by a finite periodic distribution in the dimension transverse to the direction of incidence of sound waves. This has been done by periodically replicating in the $z$-axis, a finite number of times, the system studied in Chapter 5. A periodicity equal to the height of the waveguide, $h_{w}$, is considered. This distribution provides the system with new features associated to both the presence of cavities between the HRs and the periodicity of the system in the direction perpendicular to the incidence of the waves, as well as to the finite size. The system occupies a total volume of $0.2356 \mathrm{~m}^{3}$, with an area of the cross-section of the system is $0.1571 \mathrm{~m}^{2}$. In this configuration, the volume of air confined inside the HRs is $0.0371 \mathrm{~m}^{3}$, and the cross-section area of the system is $0.0247 \mathrm{~m}^{2}$.

Two main reasons have been considered in order to choose the value of five rows in the system: $(i)$ reducing the effect of the size effects in the system to be studied in free field conditions; and $(i i)$ due to the conditions of the floor at the anechoic chamber (grid), the weight of the system is a limiting factor.

As a result of the replication (see Figure 6.1), we can see that the system is an array of HRs in 2D. Therefore, we expect to combine the effects of absorption due to resonances with the effects of diffusion associated to the effect of the cavities between the HRs and the periodicity of the system in the direction transverse to the incidence of the sound waves. The combination of both properties increases the performance of the system for the noise mitigation at the launch pad.

The system is characterized in free field conditions in anechoic chamber. In this configuration the finite size of the system has important effects on the acoustic field and can induce variations of acoustic pressure in front of the system, as will be seen later on. In this case the system is not embedded into an impedance tube and, as a consequence, plane waves cannot be assumed. Additionally, due to this fact this study has a slightly closer approach to the one of the system that will be proposed to be used at the launch pad, since it will be in open field. 
a)

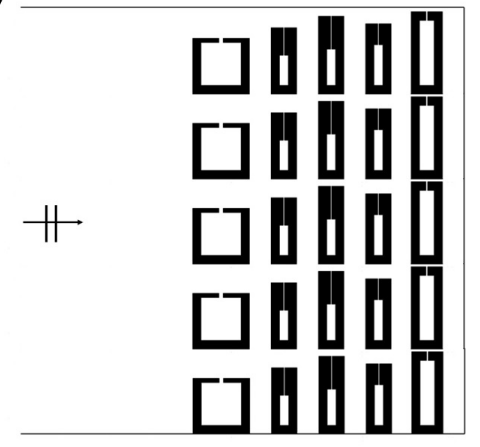

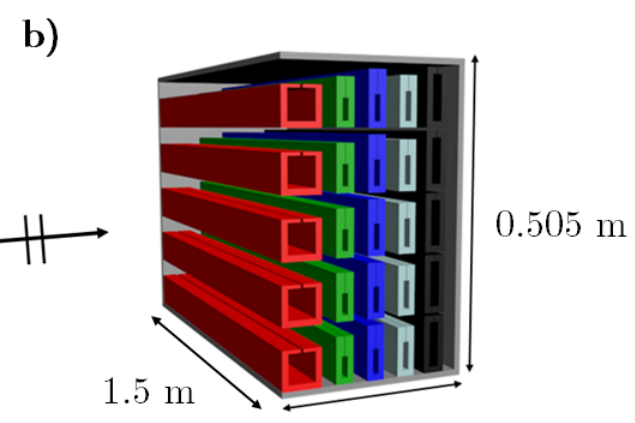

$0.311 \mathrm{~m}$

Figure 6.1: a) Cross-section (2-D view); and b) cross-section (3-D view) of the system to be studied at the anechoic chamber.

\subsection{Parametric study of finite size effects}

In general, edge effects appear due to the finite size of a given system. The interaction of the sound waves with the boundaries of the system produce diffraction that may modify the pressure field in front of the system. This plays an important role in the resulting absorption coefficient calculated from this data.

The effect of the finite size has been numerically studied by using the Finite Element Method (FEM). To do so, the system has been modeled as it is shown in Figure 6.2. The sound absorption coefficient, $\alpha$, has been obtained by placing two measurement points in front of the system at given distances and calculating the Transfer Function, $H_{12}$, between them by means of the Transfer Function Method (TFM) [109], detailed in Chapter 3.

The fundamentals of the set-up are the same of those detailed in Ref. [109] (see Figure 4.2), i.e. a sound source placed in front of the sample at a certain distance of the system of HRs and two microphones aligned between them and with the sound source and the system.

The numerical simulations have been implemented by following the same procedure than the one shown in Chapter 5. In this case, the entire domain in which sound waves propagate is surrounded by Perfectly Matched Layers (PML) to implement anechoic conditions (see Figure 6.2). PMLs are artificial absorbing layers that are commonly used in numerical calculations to simulate free field conditions. 


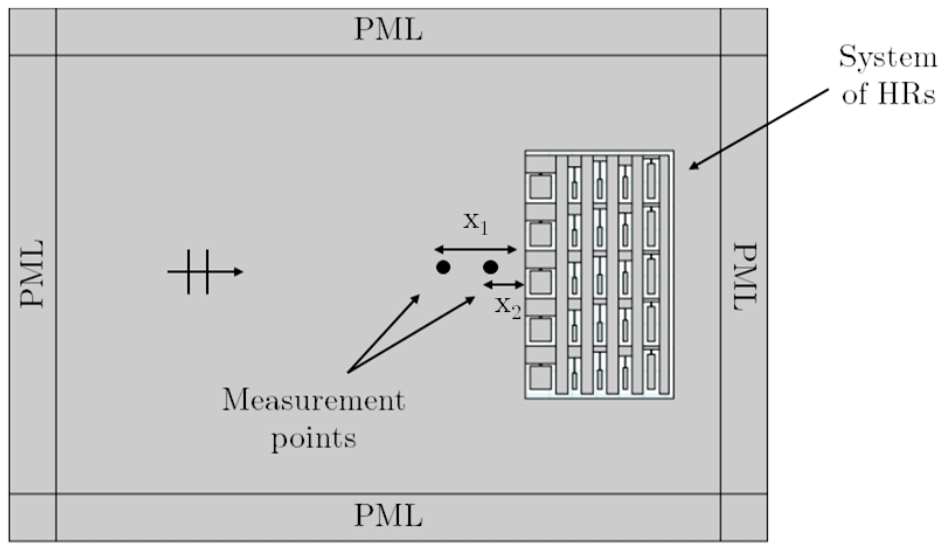

Figure 6.2: Geometry of the numerical model implemented in FEM.

Figure 6.3 shows the sound absorption coefficient obtained numerically by placing the measurement points at three different distances from the surface of the system: $x_{1}=4 \mathrm{~cm}$ and $x_{2}=1.5 \mathrm{~cm}, x_{1}=14 \mathrm{~cm}$ and $x_{2}=11.5 \mathrm{~cm}$ and $x_{1}=44$ $\mathrm{cm}$ and $x_{2}=41.5 \mathrm{~cm}$. It is important to note that the distance between the two points is equal to $2.5 \mathrm{~cm}$ in all the cases. The distance between the two microphones of $s=2.5 \mathrm{~cm}$ has been chosen based on the minimization of edge effects. Some alternatives were considered. On the one hand, increasing the distance $x_{1}$ while keeping $x_{2}$ constant increased the effect of the diffraction due to the edges of the system in a similar way as shown in Figure 6.3. On the other hand, decreasing the distance $s$ was not possible due to the size of the capsules of the microphones.

As it can be observed in Figure 6.3, the resulting $\alpha$ varies depending on this distance due to the finite size effects.

The two main consequences associated to the edge effects are:

- The bandwidth of the region in which $\alpha$ is maximum is reduced when the acoustic pressure is measured far from the system (considering the same distance between both microphones). This is due to the fact that the diffusion originated by the edges alter the pressure field and the hypothesis of the method (plane waves) is not fulfilled. The acoustic pressure field measured in front of the structure has contributions coming from the diffraction in the edge of the system. The value of the sound absorption of the finite system differs from the one of the optimized system due to this contribution. 


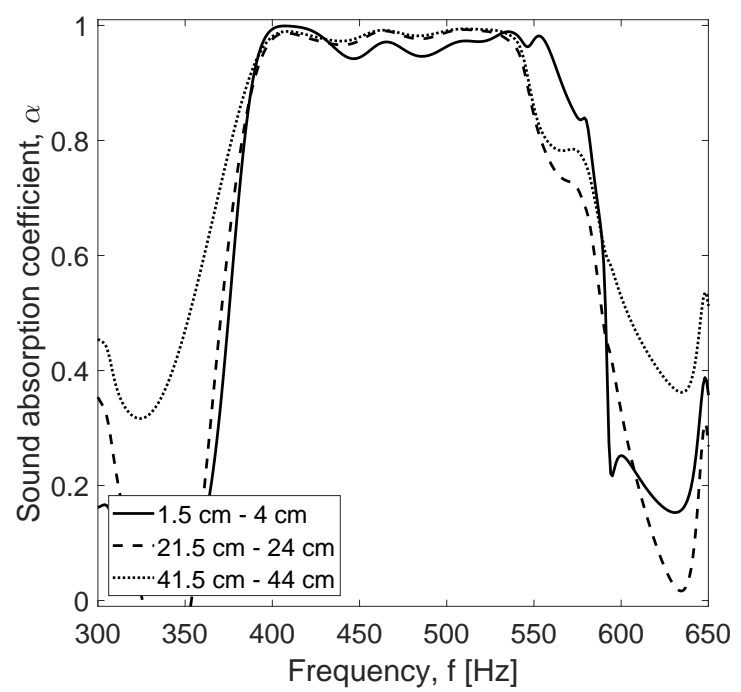

Figure 6.3: Sound absorption coefficient of the system obtained numerically by placing the measurement points at $x_{1}=4 \mathrm{~cm}$ and $x_{2}=1.5 \mathrm{~cm}$ (solid line), $x_{1}=14 \mathrm{~cm}$ and $x_{2}=11.5$ $\mathrm{cm}$ (dashed line) and $x_{1}=44 \mathrm{~cm}$ and $x_{2}=41.5 \mathrm{~cm}$ (dotted line).

- Out of the optimized range of frequencies $\alpha$ increases. Part of the acoustic waves are diffracted by the edges of the system in directions out of the region of interest in front of it, where the measurement points are placed. Since this energy is not coming back reflected by the system and is not detected in the measurement, the method wrongly assumes that is being absorbed.

These two points can be summarized as follows: since the system has a finite size and is placed in an open volume (free field), diffraction associated to the edge effects appears and scatters the acoustic field in different forms depending on the frequency. This results in the alteration of $\alpha$ calculated within the optimized range of frequencies, being reduced at some frequencies and increased for some others out of this range. The diffraction generates maximum and minimum lobes according to the direction that also depend on the frequency.

The presence of edge effects associated to the finite size of the system modifies the pressure field in front of it and could prevent the achievement of the critical coupling conditions. The alteration of the pressure field by the border effects can be minimized by choosing the measurement points that are placed close to the sample. 
absorption

\subsection{The diffusion coefficient}

The parameters that are studied in the system proposed in this Chapter are the sound absorption coefficient, $\alpha$, the insertion loss in reflection, $I L R$, and the diffusion coefficient, $d . \alpha$ and $I L R$ have been already defined and used in Chapter 5 .

In the problem that we are facing in this Chapter, the sound waves impinging a surface can be either absorbed as it has been previously described or reflected (since transmission is not considered). Furthermore, the sound energy that is reflected back can be reflected in a specular direction, following Snell's law, or in a diffuse way, spread in different directions. This spread of the sound waves is known as sound diffusion. In order to characterize this physical phenomenon, the diffusion coefficient $d$ is used.

This parameter was studied in the preliminary works, detailed in Chapter 2. Due to the periodicity in the distribution of its elements, these properties were found to be able to spread sound in frequencies around the band gap.

Following the same principle, the presence of cavities in the system of HRs increases the diffusive properties of the system with respect to a system of HRs with no cavities. In order to quantify this improvement, new optimization process has been carried out for a system of HRs with no cavities (i.e., the parameter $L$ is fixed to $L=0 \mathrm{~m}$ ). The geometrical parameters for this system are detailed in Table 6.1, and the geometry of the system can be observed in Figure 6.4.

Table 6.1: Optimized geometrical parameters obtained for each Helmholtz resonator composing the system with no cavities $(L=0 \mathrm{~m})$.

\begin{tabular}{|c|c|c|c|c|c|}
\hline Parameter [mm] & HR1 & HR2 & HR3 & HR4 & HR5 \\
\hline$h_{n}$ & 24.49 & 21.31 & 29.66 & 39.39 & 44.32 \\
\hline$L_{n}$ & 8.00 & 1.08 & 1.00 & 1.00 & 1.00 \\
\hline$h_{c}$ & 16.51 & 35.27 & 31.05 & 29.39 & 34.32 \\
\hline$L_{c}$ & 80.00 & 10.81 & 10.00 & 10.00 & 10.00 \\
\hline$h_{s}$ & 50.00 & 34.42 & 30.29 & 22.23 & 12.36 \\
\hline$L_{s}$ & 100.00 & 30.81 & 30.00 & 30.00 & 30.00 \\
\hline
\end{tabular}

We have obtained $d$ for the proposed system with cavities and the one of a system, optimized in the same frequency range, with no cavities between the elements. 


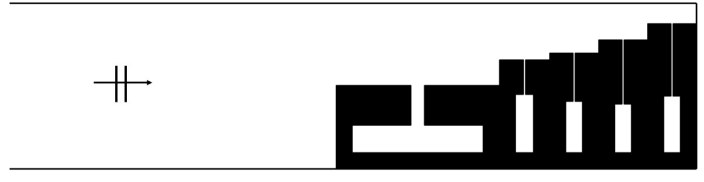

Figure 6.4: Tuned geometry of the optimized system for perfect sound absorption from 400 to $560 \mathrm{~Hz}$ with no cavities.

The diffusion coefficient has been numerically obtained for both systems by measuring the scattered far-field in a semi-circumference of radius $5 \mathrm{~m}$ centered in the sample as specified in the standard ISO 17497-2 [74]. The pressure field has been measured in a total of 180 points composing the semi-circumference, and the diffusion coefficient has been derived by using the following equation:

$$
d=\frac{\left(\sum_{i=1}^{n} 10^{S I L_{i} / 10}\right)^{2}-\sum_{i=1}^{n}\left(10^{S I L_{i} / 10}\right)^{2}}{(n-1) \sum_{i=1}^{n}\left(10^{S I L_{i} / 10}\right)^{2}},
$$

where $S I L_{i}$ is the Sound Intensity Level of the reflected sound in the $i$-th measurement position, defined as

$$
S I L_{i}=10 \log \frac{I_{i}}{I_{0}},
$$

and $n$ is the number of measurement positions.

We also define the Global Diffusion Parameter $(G D P)$ as an analogy to the Overall Sound Pressure Level $(O A S P L)$, which indicates the total energy contained in the spectrum obtained by integration over all resolved frequencies [48]. Thus, the GDP can be defined as the total diffusion coefficient obtained by integration over all resolved frequencies:

$$
G D P=\frac{\int_{f_{1}}^{f_{2}}\left(d-d_{r e f}\right) \mathrm{df}}{\Delta f}
$$

where $\Delta f=f_{2}-f_{1}$ and being $f_{1}$ and $f_{2}$ the minimum and maximum frequencies of the range of optimization. 
absorption

\subsection{Experimental framework}

Once the system has been designed and studied both analytically and numerically, the next step leads to its experimental characterization.

The system shown in Figure 6.1 has been manufactured using medium density fiber (MDF). The density of the MDF is $\rho=558 \mathrm{~kg} / \mathrm{m}^{3}$ and its specific acoustic impedance $Z=1.215 \cdot 10^{6} \mathrm{~kg} / \mathrm{s} \cdot \mathrm{m}^{2}$ [116]. Thus, this material can be considered acoustically rigid due to its high impedance compared to the one of the air $\left(Z=420 \mathrm{~kg} / \mathrm{s} \cdot \mathrm{m}^{2}\right)$, which gives the reflecting behavior that fits our goals. Furthermore, this material allows easiness in the construction process and precision manufacturing the HRs. The panels used to build the system have a thickness of $1 \mathrm{~cm}$ with the purpose of avoiding vibroacoustic effects in the structure, that may be produced by the acoustic excitation of the structures when the sound wave impinge them. The whole structure is surrounded by wood panels at all ends except the side where the incident wave is impinging on the structure.

It is important to note that in the system studied in Chapter 5, the depth of the system (in the transverse direction to the incidence of sound waves) is considered infinite due to the fact that it is confined into the tube. Nevertheless, since the measurements are carried out in free field with a system of finite size in the transverse direction, this assumption cannot be made in this case. In order to consider this dimension as much bigger than the others in the system, so the influence of the edges is minimized, it has been extruded to a depth of $1.5 \mathrm{~m}$. The system has a height of $0.525 \mathrm{~m}$ and a length of $0.311 \mathrm{~m}$.

The experimental measurements have been carried out at the anechoic chamber at the Campus of Gandia of the Universitat Politècnica de València (UPV), in Spain, shown in Figure 6.5. The effective dimensions of the chamber (length $\times$ width $\times$ height) are $6.60 \times 5.22 \times 3.60 \mathrm{~m}$, corresponding to an effective volume of $124.03 \mathrm{~m}^{3}$. This room has a cut-off frequency, from which it can be considered as acoustically anechoic, of $70 \mathrm{~Hz}$ [117].

In this experiments, the goal is to characterize the performance of the proposed system for sound absorption. The methodology used to obtain the absorption coefficient follows the same guidelines as the Transfer Function Method (TFM). The particularity of the measurements carried out in the free field conditions with respect to the TFM is that the system is not confined into a closed tube and the sound waves are not guided. Thus, in order to assume the incidence of plane waves in the system the sound source (loudspeaker) must be placed at a minimum distance from the system. 


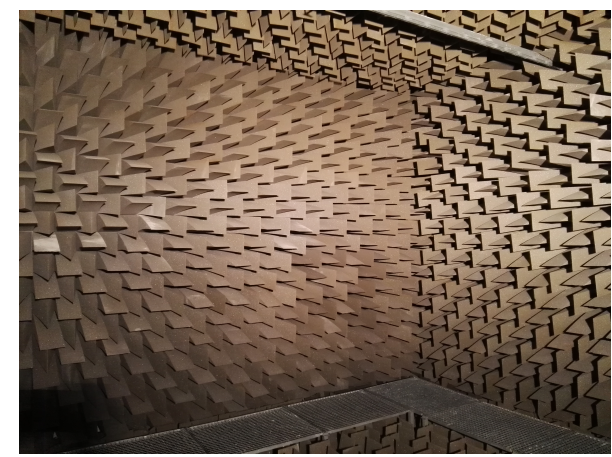

Figure 6.5: Anechoic chamber at the Campus of Gandia of the Polytechnic University of Valencia (UPV).

The sound source used to emit the signal is a loudspeaker Genelec $8030 \mathrm{~A}$ with a radius $r=6.35 \mathrm{~cm}$, frequency response from 60 to $12000 \mathrm{~Hz}$, sensitivity of $89 \mathrm{~dB}$ referred to $1 \mathrm{~W} / 1 \mathrm{~m}$ and a maximum acoustic pressure of $107 \mathrm{~dB}$ at 1 $\mathrm{m}$ of distance. Two Brüel \& Kjær 4189-A-021-1/2-inch free field microphones with Type 2671 preamplifiers have been used to measure the acoustic pressure. These microphones have a frequency response from 20 to $20000 \mathrm{~Hz}$, and have been calibrated with a sensitivity of $47.2 \mathrm{mV} / \mathrm{Pa}$ and $54.3 \mathrm{mV} / \mathrm{Pa}$, respectively, for a pure tone at a frequency of $1 \mathrm{kHz}$ with a sound pressure level of $94 \mathrm{~dB}$.

The finite size of the system produces diffraction due to edges, as it has been described in Section 6.1. In order to minimize these effects, the two microphones have been placed at a distance of $x_{1}=4 \mathrm{~cm}$ and $x_{2}=1.5 \mathrm{~cm}$. This proximity minimizes the effect of diffraction, as it was studied in Section 6.1.

The distance between the source and the sample is also important, since the experimental solution at low frequencies has been found to be more deviated from the simulations when the source is placed closer to the surface of the sample [118]. If the sound source is set close to the system, the measurement may lose the validity of the plane wave assumption [119]. Thus, the sound source has been placed at a distance of $3 \mathrm{~m}$ of the system of HRs, since this distance is sufficient to allow the incidence of almost plane waves in the system. In order to avoid unwanted reflections to the acoustic field coming from the grating covering the floor of the anechoic chamber, the system has been placed at a height of $1.5 \mathrm{~m}$. A picture of the complete experimental set-up can be seen in Figure 6.6. 
absorption

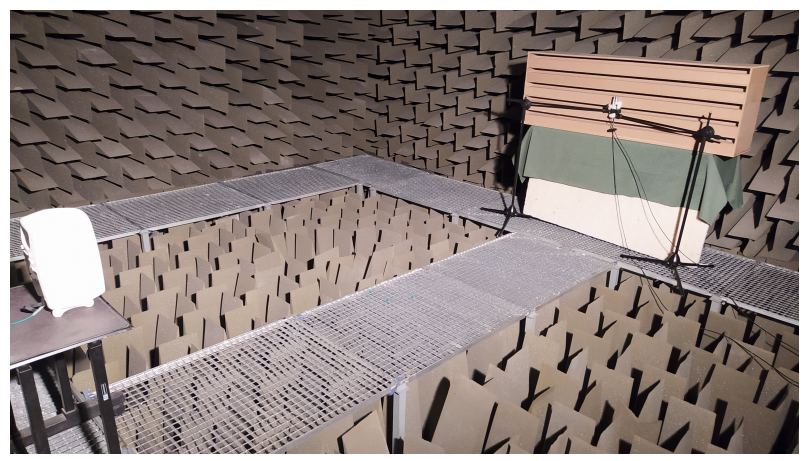

Figure 6.6: Experimental set-up of the system placed inside the anechoic chamber.

\subsection{Results and discussion}

A system composed of a distribution of five HRs replicated in the direction transverse to the propagation of the waves has been analyzed. The study of the system has been carried out in free field conditions, and three parameters have been obtained: sound absorption coefficient, $\alpha$, diffusion coefficient, $d$, and Insertion Loss in Reflection, $I L R$.

\subsubsection{Sound absorption coefficient}

The sound absorption coefficient of the system has been obtained analytically (TMM), numerically (FEM) and experimentally (TFM). Figure 6.7 shows the result obtained by means of the three methods. A broad range of frequencies with a sound absorption coefficient above 0.9 can be observed. This absorption is associated to the effect of the resonances of the different elements of the system and the tuning of the viscothermal losses in order to fulfill the critical coupling condition.

A good agreement can be observed in Figure 6.7 for the numerical and experimental results in the range of frequencies. The results shown obtained with the TMM (solid line) slightly differs in the magnitude of $\alpha$ from the other two due to the fact that it is a 1-D model that considers the propagation of waves only in the $x$ direction, a situation that is closer to the system embedded in the tube but not for the system in free filed conditions. Thus, it is worth to say here that we have included the sound absorption coefficient obtained with this method in order to establish a comparison between the TMM, used to design the system, and the FEM and experimental measurements. 


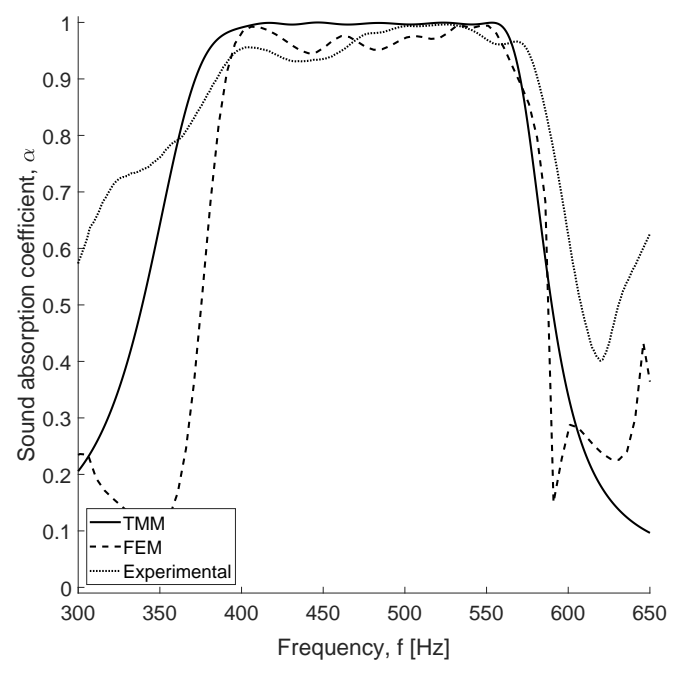

Figure 6.7: Sound absorption coefficient of the system in the anechoic chamber, obtained with the TMM (solid line), FEM (dashed line) and experimentally (dotted line).

The information shown in the figure can be represented in form of a table in order to obtain, in a more intuitive way, the bandwidth of the absorption coefficient. Table 6.2 shows the ranges of frequencies in which the sound absorption with $\alpha \geq 0.9, \alpha \geq 0.95$ and $\alpha \geq 0.99$. The values of the absorption coefficient have been obtained analytically, numerically and experimentally. In Table 6.3 , we can observe that the absorption coefficient is greater that 0.9 in a bandwidth close to $200 \mathrm{~Hz}$ for the analytical and experimental studies.

For the case of $\alpha \geq 0.99$, discrepancies are more evident, and while the bandwidth is of $162 \mathrm{~Hz}$ for the analytical model, it is reduced to $22 \mathrm{~Hz}$ and 42 $\mathrm{Hz}$ for the numerical and experimental results, respectively. This is due to the fact that, the more the threshold of $\alpha$ is increased the more difficult is to fulfill the conditions to achieve it. As a consequence, an unbalance between the leakage and losses in the system can be easily produced by many factors, such as imprecision in manufacturing the system.

If we look at the representation of the reflection coefficient in the complex frequency plane shown in Chapter 5 (Figure 5.10), it is possible to observe that the range of maximum $\alpha$ is in the same frequency range for which it was optimized. 
absorption

Table 6.2: Ranges of frequencies with sound absorption higher than $0.9,0.95$ and 0.99 .

\begin{tabular}{|l|c|c|c|}
\hline & $\boldsymbol{\alpha}>\mathbf{0 . 9}$ & $\boldsymbol{\alpha}>\mathbf{0 . 9 5}$ & $\boldsymbol{\alpha}>\mathbf{0 . 9 9}$ \\
\hline TMM & $372-570 \mathrm{~Hz}$ & $380-566 \mathrm{~Hz}$ & $399-561 \mathrm{~Hz}$ \\
\hline FEM & $396-553 \mathrm{~Hz}$ & $439-531 \mathrm{~Hz}$ & $485-503 \mathrm{~Hz}$ \\
& & $534-549 \mathrm{~Hz}$ & $541-545 \mathrm{~Hz}$ \\
\hline EXP & $385-581 \mathrm{~Hz}$ & $398-416 \mathrm{~Hz}$ & $495-537 \mathrm{~Hz}$ \\
& & $461-575 \mathrm{~Hz}$ & \\
\hline
\end{tabular}

Table 6.3: Total bandwidth $(\Delta f)$ of the peaks of sound absorption higher than $0.9,0.95$ and 0.99 .

\begin{tabular}{|l|c|c|c|}
\hline & $\boldsymbol{\alpha}>\mathbf{0 . 9}$ & $\boldsymbol{\alpha}>\mathbf{0 . 9 5}$ & $\boldsymbol{\alpha}>\mathbf{0 . 9 9}$ \\
\hline TMM & $198 \mathrm{~Hz}$ & $186 \mathrm{~Hz}$ & $162 \mathrm{~Hz}$ \\
\hline FEM & $157 \mathrm{~Hz}$ & $107 \mathrm{~Hz}$ & $22 \mathrm{~Hz}$ \\
\hline EXP & $196 \mathrm{~Hz}$ & $132 \mathrm{~Hz}$ & $42 \mathrm{~Hz}$ \\
\hline
\end{tabular}

\subsubsection{Diffusion coefficient}

The hypothesis previous to the performance of the study is that the presence of cavities between the HRs, as well as the periodic distribution in height of the system, increase its diffusive properties. In order to study this effect, the diffusion coefficient, $d$, of the system has been numerically obtained and compared with the one of a system without cavities.

In order to obtain the geometry of the system without cavities, a new optimization has been carried out in order to tune the geometry of the HRs to have perfect sound absorption in the same range of frequencies (from 400 to $560 \mathrm{~Hz}$ ) than the system with cavities. In this case, we fix the values of the cavities between the HRs as $L=0 \mathrm{~m}$ (see Figure 3.16 in Chapter 3) to suppress this distance. This new optimization has been performed in order to ensure that both systems are studied under the same conditions for sound absorption and to analyze the influence of the presence of cavities in the value of $d$.

Figure 6.8 shows the diffusion coefficient, $d$, of a system without cavities and the system with cavities that was proposed at the beginning of this Chapter, both optimized to achieve perfect sound absorption in the same frequency range. The cavities introduce additional modes in the system of diverse nature. These modes mainly appear in the direction perpendicular to the incidence of the wave into the structure and are associated to coupled modes of a cavity with the adjacent HRs, the resonance of each HR and the height of the system. These additional modes can be seen in the insets of Figure 6.8, showing 
new nodal lines introduced by the cavities and the transverse modes. As it can be observed in the insets, the modes are not symmetrical in the direction transverse to the incidence of the sound wave in the system and lead to transverse variations of the acoustic pressure field, increasing the sound diffusion consequently.

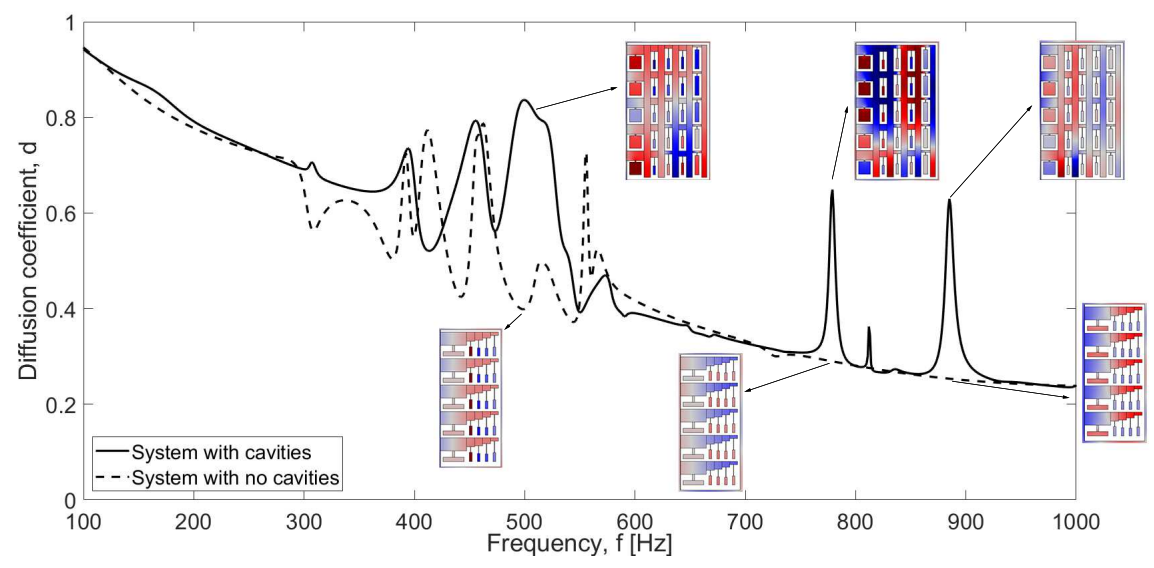

Figure 6.8: Diffusion coefficient of the system obtained numerically in the free field for the proposed system of HRs with cavities (solid line) and a system optimized for the same range of frequencies with no cavities between the HRs (dashed line).

In the frequency range in which $d$ is represented in the Figure 6.8, from 100 to $1000 \mathrm{~Hz}$, the GDP of the systems without and with cavities are 0.4879 and 0.5259 , respectively. This shows a slight increase in $d$ due to the presence of air cavities between the HRs and the additional modes introduced by them.

Additionally, if the attention is focused specifically in the range of frequencies in which the optimization has been implemented, the system without cavities changes to $G D P=0.5297$, while the one of the system with cavities rises up to $G D P=0.6461$.

\subsubsection{Insertion Loss in Reflection}

The value of the Insertion Loss in Reflection $(I L R)$, previously defined in Chapter 5 , has a close relation with that of the sound absorption and diffusion coefficients. The study of the $I L R$ gives an estimation of these two phenomena together since the $S P L$ at a given point or region in front of the structure is related to both of them. This parameter compares the sound pressure level $(S P L)$ 
absorption

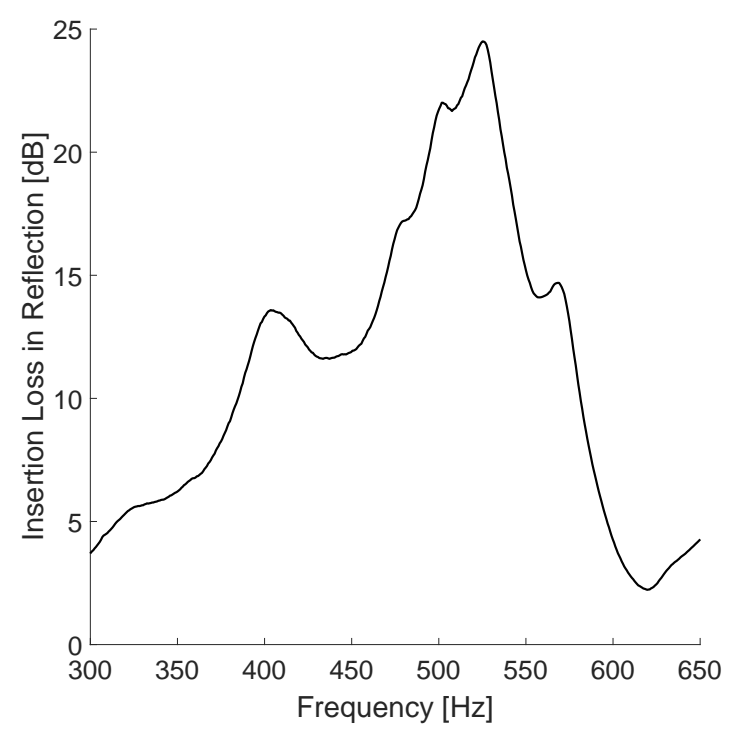

Figure 6.9: Insertion Loss in Reflection of the system obtained experimentally in the freefield for the system of HRs.

in front of the system with the one of a flat rigid surface. As a consequence, a high absorption is translated in a lower $S P L$ in front of the structure.

The ILR has been experimentally obtained by following the same procedure than in Chapter 5, a flat rigid surface has been considered as a reference case.

The resulting $I L R$ in the frequency range in which the system has been optimized are shown in Figure 6.9 are shows. As it was initially expected due to the sound absorption coefficient in this range of frequencies, the peak of the $I L R$ is high, reaching an average value of $16.5 \mathrm{~dB}$.

\subsection{Conclusions}

In this Chapter a system composed by a distribution of five HRs separated by air cavities has been studied. Keeping the optimized geometry of the system designed in Chapter 5, the system has been replicated in height and numerically and experimentally studied in free field conditions. 
The influence of the size effects in the resulting sound absorption coefficient has been analyzed by placing measurement points at different distances from the system. When these points are placed close enough, these effects are minimized and it is possible to measure the pressure field given by the interaction of the sound waves with the system.

The behavior of the system has been characterized by means of the study of three parameters: the sound absorption coefficient, $\alpha$, the diffusion coefficient, $d$, and the Insertion Loss in Reflection, $I L R$.

The absorption of the system is maximum in the frequency range for which the optimization has been carried out (from 400 to $560 \mathrm{~Hz}$ ). This means that the system keeps the absorptive properties when it is extrapolated from the distribution embedded into an impedance tube (studied in Chapter 5) to free field conditions.

In this work we have also studied the contribution of the cavities in the system to the modification of the diffusion coefficient. A higher value in $d$ has been reported in a system in which the HRs are separated by air cavities with respect to a system optimized in the same frequency range in which no cavities are considered. This is due to the fact that these cavities introduce additional modes in the system.

The third parameter studied is the $I L R$, which involves the two that have been previously mentioned since it shows, in a general way, the reduction in the sound pressure level. In this case, as it was expected, the $I L R$ has a high value in all the range of the optimization, with an average value of $16.5 \mathrm{~dB}$. Comparing this result with the one that was presented for sonic crystals in Chapter 2, one can see that the $I L R$ is much greater in the case of the HRs, mainly due to the higher absorption coefficient of these systems with respect to SCs, but also to the influence of their diffusive properties.

The system presents a high peak in the $I L R$ in the optimized range of frequencies, which makes it an interesting system to continue being studied for applications in the aerospace field. 



\section{Chapter 7}

\section{Conclusions and future work}

\subsection{Conclusions}

In this Thesis a proof of concept of a system for the mitigation of the effects of the noise generated during the ignition and first stages of the lift-off of spacecrafts has been developed. In this context, a system composed by Helmholtz resonators (HRs) separated by air cavities has been designed for perfect sound absorption and diffusion. To achieve this goal, we have made use of the critical coupling condition, based on the balance between the leakage of energy and the inherent losses in the system.

A literature research has been carried out in order to understand the nature of the real problem, as well as the previous works that have been already developed. Most of the solutions that are being studied and applied nowadays are based on the use of water deluge systems and have limitations both in the range of frequencies and in the magnitude of the noise mitigation associated to the effect of water.

The fundamentals of the TMM and the critical coupling condition have been also studied and applied to the design of perfect absorbers. These tools have been used for designing a system of HRs in order to perfectly absorb sound waves from 400 to $560 \mathrm{~Hz}$. The geometry of the HRs has been tuned by using an optimization algorithm based on the TMM and implemented using 


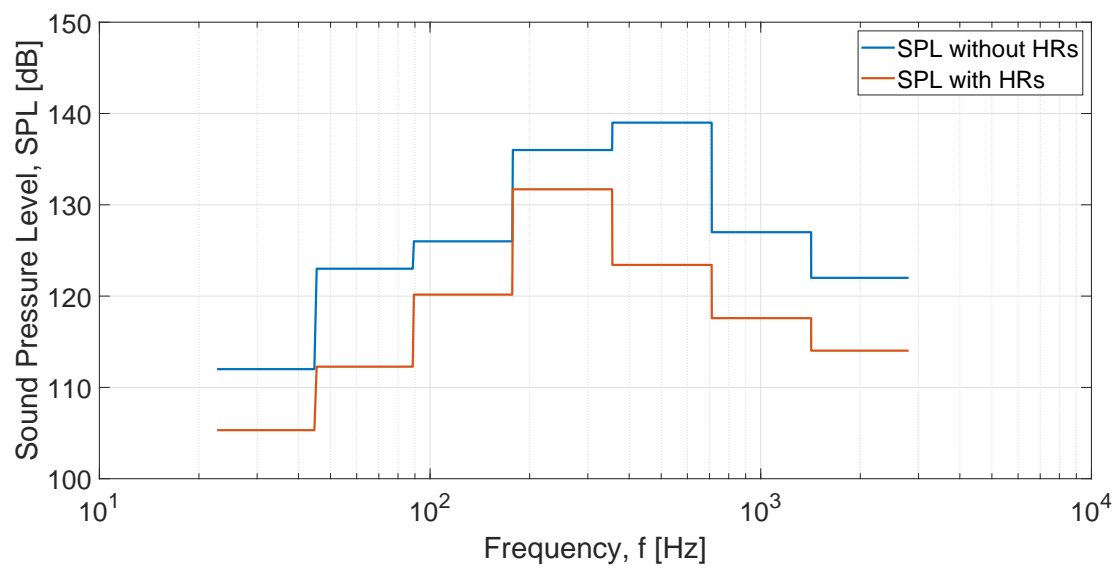

Figure 7.1: Spectrum of the $S P L$, integrated in octave frequency bands, of the lift-off phase of the rocket VEGA C without (solid blue) and with (solid red) the system of HRs.

a SQP method. The system has been studied analytically, numerically and experimentally, and it has shown a good performance for sound absorption in the optimized range of frequencies, with a sound absorption coefficient, $\alpha$, higher than 0.9 in a bandwidth of $200 \mathrm{~Hz}$.

The diffusive properties of the system have been also studied. The influence of the cavities placed behind the HRs has been analyzed by comparing the diffusion coefficient, $d$, of the proposed system with cavities with that of a system optimized for the same frequency range in which cavities are not considered. The Global Diffusion Parameter, GDP, obtained is 0.5297 for the system without cavities, while the value in the system with cavities is increased up to 0.6461 .

The hypothetical reduction in the $S P L$ associated to these two physical phenomena has been theoretically quantified by means of the ILR. Figure 7.1 shows, in blue, the spectrum (in octave bands and in decibels) of the limit level regarding the $S P L$ that the rocket VEGA C can stand during the lift-off. This spectrum has been obtained from Ref. [111]. Considering the assumptions and limitations of this study (i.e., linear regime, plane waves and normal incidence of the sound waves), the theoretical difference between this level and the one obtained after placing the system of HRs that has been designed in this Thesis has been calculated. 
As it can be observed in the figure, the $S P L$ is reduced in the entire range of frequencies (from $20 \mathrm{~Hz}$ to $2828 \mathrm{~Hz}$ ). This reduction of the $S P L$ is much more evident in the octave frequency band from $353.6 \mathrm{~Hz}$ to $707.1 \mathrm{~Hz}$, since the range of frequencies for which the system of HRs has been optimized is within this range.

The Overall Sound Pressure Level, OASPL, is reduced from $141.2 \mathrm{~dB}$ to 117.7 $\mathrm{dB}$ by using the proposed system, which means an overall reduction of $23.5 \mathrm{~dB}$ in the studied range of frequencies. This value expressed in terms of energy reduction reaches the value of $99.5 \%$, meaning that approximately half of the acoustic energy is reduced.

\subsection{Future work}

The work developed during this Thesis has been carried out assuming plane (or almost plane, in the case of the study performed in Chapter 6) waves with normal incidence in linear regime, setting the first steps to the development of a system based on HRs to be used for mitigating noise in aerospace applications. Keeping in mind the limitations of the study regarding to the real case, the system showed promising results regarding to sound absorption and diffusion, which makes feasible its study in more depth.

The future work lines presented here are divided as a function of its field of application, considering applications in Engineering and in scientific studies.

\subsubsection{Applications in Engineering}

- Analyze the behavior of the system considering non-plane waves. This includes, for example, spherical waves or considering monopolar sources. This would show the consistency of the system and would give and idea of its performance in a slightly more complex situation in which the input acoustical parameters are still under control.

- Study the effectiveness of the system with random incidence. The incidence of the sound waves generated during the ignition of the spacecraft can be considered as normal in a simplification of the problem. Nevertheless, the reflections in the launch pad and surrounding structures may cause a diffuse field. The study of conditions similar to these ones can be done by carrying out experimental measurements in a reverb chamber, 
like the one at the Campus of Gandia of the Universitat Politècnica de València.

- Consider more realistic sound pressure levels. This could be done by using loudspeakers able emit higher pressure levels or using a scaled mock-up of a spacecraft's engine. This last step would require manufacturing the system of HRs in a material that can stand high temperatures and more extreme conditions, since the MDF is highly flammable and not suitable to be used in humid environments. Some interesting materials for this stage that fulfill these requirements are concrete and steel.

- Using more realistic pressure/time signal from flight measurements or from CFD simulations. Considering more realistic signals as an input for the system would give a more accurate approach to the real problem and would allow a better tuning of the system regarding to the existing needs.

\subsubsection{Scientific studies}

- Study of the nonlinear effects in the system associated to the high pressure field generated during the lift off. This study is necessary given the nature of the real problem, in which an extreme environment is generated. Nonlinear effects may modify the performance of the system in a different degree depending on the strength of the nonlinearity, so their influence must be taken into account for the final application. Some of the sources of nonlinearities in the problem that has been studied are:

- Nonlinearities in the HRs. Non-linearities can be produced due to the nonlinear response of the HRs. This response can also induce perfect absorption if the critical coupling condition is fulfilled in these conditions. Nonlinearities in HRs are connected with flow separation and vorticity in the neck, and present different behavior depending on the detailed geometrical characteristics of the edges of the neck [120].

- Nonlinear propagation in fluids. The points in the wave with the largest values of condensation travel most rapidly, leading to the development of a distortion in the wave [121]. 
- Nonlinearities of the fluid flow. The flow of air going through a narrow duct can set that duct into oscillation. This may cause vorticity and turbulent motion of the medium [121].

\section{- Nonlinearities in the sound source.}

- Combine plates and Helmholtz resonators. There are several reasons to consider both HRs and plates in the system:

- The combination of resonating elements of different nature adds degrees of freedom (DoF) to the design of the system with respect to those configurations in which only one of these elements is considered and, therefore, the possibilities in the design increase.

- The physical phenomena involved in the sound absorption would include the viscoelastic losses in the plates in addition to the viscothermal losses in the HRs that have been studied in this Thesis.

- From the constructive point of view, the consideration of plates in the system can give more variability in the use of materials and, depending on the configuration resulting from the optimization process, a reduction in the space occupied by the system.

This project will have continuity under the Technology Research Programme (TRP) "Launch Sound Level Reduction", in which the Universitat Politècnica de València will take part. The aim of this activity is to propose, design, implement and experimentally validate a sound mitigation method, applicable to a real launch configuration, which results in a significant decrease of the sound pressure levels generated in the launch area during the lift-off of spacecrafts, as well as the reduction of the acoustic loads experienced by the spacecraft inside the fairing. 



\section{Bibliography}

[1] J. P. Arenas and R. N. Margasahayam.

"Noise and vibration of spacecraft structures".

In: Revista chilena de ingeniería 14.3 (2005), pp. 251-264

(cit. on p. 1).

[2] J. Panda, R. N. Mosher, and B. J. Porter. "Noise source identification during rocket engine test firings and a rocket launch".

In: Journal of Spacecraft and Rockets 51.6 (2014), pp. 1761-1772 (cit. on pp. 2, 3).

[3] C. P. Lubert.

"From Sputnik to SpaceX: Sixty years of rocket launch acoustics". In: Acoustics Today 14.4 (2018), pp. 38-46 (cit. on pp. 2, 10).

[4] R. W. Hess, R. W. Fralich, and H. H. Hubbard.

Studies of structural failure due to acoustic loading. Tech. rep.

National Advisory Comittee for Aeronautics, 1957 (cit. on p. 2).

[5] K. M. Eldred. Acoustic loads generated by the propulsion system. Tech. rep.

National Aeronautics and Space Administration (NASA), 1971 (cit. on pp. 2, 8, 9). 
[6] J. K. Ignatius, S. Sathiyavageeswaran, and S. R. Chakravarthy. "Hot-flow simulation of aeroacoustics and suppression by water injection during rocket liftoff".

In: AIAA Journal 53.1 (2015), pp. 235-245 (cit. on pp. 3, 11, 12).

[7] G. Elias.

"Ariane 5 at lift off: localization and ranking of acoustic sources". In: 29 ${ }^{\text {th }}$ International Congress and Exhibition on Noise Control Engineering (InterNoise 2000) (cit. on pp. 3,6).

[8] D. Gély et al.

"Acoustic environment of the VEGA launch vehicle at lift-off".

In: Forum Acusticum 2005 (cit. on pp. 3, 7, 14, 15, 18).

[9] J. K. Ignatius et al. "Suppression of jet noise by staged water injection during launch vehicle lift-off".

In: International Journal of Aeroacoustics 7.3-4 (2008), pp. 223-242 (cit. on pp. 4, 12).

[10] H. T. Nagamatsu, R. E. Sheer Jr., and E. C. Bigelow.

Subsonic and supersonic jet flow and acoustic characteristics and supersonic suppressors. Tech. rep.

National Aeronautics and Space Administration (NASA), 1972 (cit. on p. 3).

[11] S. Tsutsumi et al.

"Effect of deflector shape on acoustic field of launch vehicle at lift-off". In: $4^{\text {th }}$ AIAA Aerospace Sciences Meeting (cit. on pp. 4, 9, 17).

[12] D. Papamoschou and M. Debiasi.

"Directional suppression of noise from a high-speed jet".

In: AIAA Journal 39.3 (2001), pp. 380-387 (cit. on p. 4).

[13] S. Saravanana et al. "Experimental investigation of flow and noise characteristics of impinging twin jets, simulating starting flow from a rocket booster on a launch pad". In: National Conference on Space Transportation Systems: Opportunities and Challenges (cit. on p. 4). 
[14] C. K. W. Tam. "Supersonic jet noise".

In: Annual Review of Fluid Mechanics 27 (1995), pp. 17-43 (cit. on p. 4).

[15] J. M. Seiner. "Advances in high speed jet aeroacoustics".

In: AIAA Pap. 84.2275 (1984) (cit. on pp. 4, 5).

[16] D. C. Allgood, G. P. Saunders, and L. A. Langford.

Reduction of altitude diffuser jet noise using water injection. Tech. rep. National Aeronautics and Space Administration (NASA), 2014 (cit. on pp. 4, 12).

[17] T. D. Norum. "Screech suppression in supersonic jets".

In: AIAA Journal 21.2 (1983), pp. 235-240 (cit. on p. 6).

[18] J. Panda, R. N. Mosher, and B. J. Porter.

Identification of noise sources during rocket engine test firings and a rocket launch using a microphone phased-array. Tech. rep.

National Aeronautics and Space Administration (NASA), 2013 (cit. on p. 6).

[19] P: Malbéqui, R. Davy, and C. Bresson. "Experimental characterization of the acoustics of the future Ariane 6 launch pad". In: rth $^{\text {th }}$ European Conference for Aeronautics ad Space Sciences (EUCASS).

Krakow (Poland), 2015 (cit. on pp. 6, 16).

[20] R. Venkatraman et al. "Investigation on the ignition over pressure related to launch vehicle at lift-off".

In: Indian Journal of Science and Technology 7.1 (2014), pp. 86-94 (cit. on p. 7).

[21] D. Casalino et al. "Hybridal emprirical/computational aeroacoustic methodology for rocket noise modelling".

In: AIAA J. 47 (2009), pp. 1445-1460 (cit. on p. 8).

[22] S. Candel. "Analysis of the sound field radiated by Ariane launch vehicle during lift-off".

In: La Recherche Aerospatiale: Bulletin Bimestrel de L'Office National d'Etudes et des Recherches Aerospatiales (1983), pp. 17-33 (cit. on p. 8). 
[23] J. Varnier and W. Raguenet. "Experimental characterization of the sound power radiated by impinging supersonic jets".

In: AIAA J. (2002), pp. 825-831 (cit. on p. 8).

[24] J. Varnier.

"Simplified approach of jet aerodynamics with a view to acoustics".

In: AIAA J. (2006), pp. 1690-1694 (cit. on p. 8).

[25] M. Barbarino et al. "Hybrid BEM/empirical approach for scattering of correlated sources in rocket noise prediction".

In: Journal of Sound and Vibration 403 (2017), pp. 90-103 (cit. on pp. 8, 9).

[26] C. S. Lubert. "Sixty years of launch vehicle acoustics". In: Proceedings of Meetings on Acoustics, Vol. 31, 040004.

[27] D. Casalino et al.

"Aircraft noise reduction technologies: a bibliographic review".

In: Aerospace Science and Technology 12 (2008), pp. 1-17 (cit. on p. 10).

[28] Y. Marchesse, Y. Gervais, and H. Foulon.

"Water injection effects on hot supersonic jet noise".

In: C. R. Mecanique 330 (2002), pp. 1-8 (cit. on p. 10).

[29] M. Kandula.

"Broadband shock noise reduction in turbulent jets by water injection". In: Applied Acoustics 70 (2009), pp. 1009-1014 (cit. on p. 10).

[30] V. Moriniere, Y. Gervais, and J.-L. Peube. "Numerical calculation of spectrum emission and jet noise reduction by injection of mass".

In: Proceedings of InterNoise 1995 (cit. on p. 10).

[31] E. Zoppellari and D. Juvé. "Reduction of jet noise by water injection". In: $3^{\text {rd }}$ AIAA/CEAS Aeroacoustics Conference (cit. on pp. 11, 14).

[32] L. E. Kinsler et al.

Fundamentals of Acoustics. Theory, analysis and applications. $4^{\text {th }}$ ed. (cit. on pp. 11, 12, 46, 66). 
[33] M. Kandula. Spectral attenuation of sound in dilute suspensions with nonlinear particle relaxation. Tech. rep.

National Aeronautics and Space Administration (NASA), 2008

(cit. on pp. 11, 14).

[34] T. Castelain et al. "Jet-noise reduction by impinging microjets: an acoustic investigation testing microjet parameters".

In: AIAA Journal 46.5 (2008), pp. 1081-1087 (cit. on p. 12).

[35] S. Sankaran et al.

"Suppresion of high Mach number rocket jet noise by water injection".

In: Journal of Spacecraft and Rockets 46.6 (2009), pp. 1164-1170

(cit. on p. 12).

[36] J. K. Ignatius, S. Sankaran, and S. R. Chakravarthy. "Supersonic jet noise and suppression characteristics during launch vehicle liftoff". In: $13^{\text {th }}$ Asian Congress of Fluid Mechanics (cit. on p. 12).

[37] M. Kandula. "Sound propagation in saturated gas-vapor-droplet suspensions with droplet evaporation and nonlinear relaxation". In: J. Acoust. Soc. Am. 131.6 (2012), EL434-EL440 (cit. on p. 12).

[38] B. Greska and A. Krothapalli. "Jet noise reduction using aqueous microjet injection". In: $10^{\text {th }}$ AIAA/CEAS Aeroacoustics Conference (cit. on pp. 12, 14).

[39] A. M. Vorobyov et al.

"Problem of intensity reduction of acoustic fields generated by gas-dynamic jets of motors of the rocket-launch vehicles at launch". In: Acta Astronautica 109 (2015), pp. 264-268 (cit. on p. 12).

[40] T. D. Norum.

"Reductions in multi-component jet noise by water injection". In: $10^{\text {th }}$ AIAA/CEAS Aeroacoustics Conference (cit. on p. 12).

[41] I. Herrero-Durá et al.

"Experimental study of sound attenuation by layers of water droplets". In: $10^{\text {th }}$ European Congress and Exhibition on Noise Control Engineering (EuroNoise 2015) (cit. on p. 12). 
[42] A. Krothapalli et al. "Turbulence and noise suppression of a high-speed jet by water injection".

In: J. Fluid Mech. 491 (2003), pp. 131-159 (cit. on pp. 12-14).

[43] B. Henderson. "Fifty years of fluidic injection for jet noise reduction". In: International Journal of Aeroacoustics 9.1-2 (2010), pp. 91-122 (cit. on p. 14).

[44] D. Gély and D. Galli.

"Acoustic environment of the VEGA launch vehicle at lift-off: A trade-off analysis on different launch pad configurations".

In: $11^{\text {th }}$ International Congress on Sound and Vibration (cit. on p. 15).

[45] G. Madhan Kumar, S. Senthil Kumar, and Dr. P. Maniarasan. "Effect of various deflectors on acoustic load distribution during rocket vehicle launch". In: International Journal of Engineering Research 83 Technology (IJERT) 2.6 (2013), pp. 2833-2837 (cit. on pp. 17, 31).

[46] R. Picó et al. "Acoustic behavior of the VEGA launch pad environment". In: EuroRegio 2016. Porto, Portugal, 2016 (cit. on pp. 18, 28).

[47] R. Martínez-Sala et al. "Sound attenuation by sculpture". In: Nature 378 (1995), p. 241 (cit. on pp. 18, 33, 34).

[48] Univertat Politècnica de València.

Sonic crystals for noise reduction at the launch pad. Tech. rep. Univertat Politècnica de València, 2016 (cit. on pp. 18, 33, 34, 42, 107).

[49] L. M. Garcia-Raffi et al.

"Broadband reduction of specular reflections by using sonic crystals: A proof of concept for noise mitigation in aerospace applications". In: Aerospace Science and Technology 73 (2018), pp. 300-308 (cit. on pp. 18, 33, 39, 87).

[50] Y. Duan et al. "Theoretical requirements for broadband perfect absorption of acoustic waves by ultra-thin elastic meta-films". In: Scientific Reports 5.12139 (2015) (cit. on p. 19). 
[51] T. J. Cox and P. D'Antonio. Acoustic absorbers and diffusers. 3rd ed. CRC Press, 2016 (cit. on p. 19).

[52] V. Leroy et al.

"Superabsorption of acoustic waves with bubble metascreens".

In: Physical Review B 91.020301 (2015) (cit. on p. 19).

[53] V. Romero-García et al. "Perfect and broadband acoustic absorption by critically coupled sub-wavelength resonators".

In: Scientific Reports 6.19519 (2016) (cit. on pp. 19-21, 52, 63, 83).

[54] N. Jiménez et al.

"Rainbow-trapping absorbers: Broadband, perfect and asymmetric sound absorption by subwavelength panels for transmission problems". In: Scientific Reports 7.13595 (2017) (cit. on pp. 19, 22, 23, 46, 83, 86).

[55] V. Romero-García et al. "Use of complex frequency plane to design broadband and sub-wavelength absorbers".

In: J. Acoust. Soc. Am. 139 (2016), pp. 3395-3403

(cit. on pp. 19, 20, 76, 83, 86).

[56] A. Yariv. "Universal relations for coupling of optical power between microresonators and dielectric waveguides".

In: Electrononics Letters 36.321 (2000) (cit. on p. 20).

[57] K. Bliokg et al.

"Unusual resonators: Plasmonics, metamaterials, and random media".

In: Reviews of Modern Physics 80.1201 (2008) (cit. on pp. 20, 85).

[58] N. Jiménez et al. "Ultra-thin metamaterial for perfect and quasi-omnidirectional sound absorption".

In: Applied Physics Letters 109.121902 (2016)

(cit. on pp. 20, 21, 52, 66, 70, 71, 85, 86).

[59] N. Jiménez et al.

"Quasiperfect absorption by subwavelength acoustic panels in transmission using accumulation of resonances due to slow sound". In: Physics Review B 95.014205 (2017) (cit. on pp. 20, 22, 68). 
[60] E. Yablonovith. "Inhibited Spontaneous Emission in Solid-State Physics and Electronics".

In: Physical Review Letters 58.20 (1987), pp. 2059-2062 (cit. on p. 33).

[61] S. John. "Strong Localization of Photons in Certain Disordered Dielectric Superlattices".

In: Physical Review Letters 58.23 (1987), pp. 2486-2489 (cit. on p. 33).

[62] M. S. Kushwaha et al.

"Acoustic Band Structure of Periodic Elastic Composites".

In: Physical Review Letters 71.13 (1993), pp. 2022-2025

(cit. on pp. 33, 34).

[63] J. V. Sánchez-Pérez et al.

"Sound Attenuation by a Two-Dimensional Array of Rigid Cylinders". In: Physical Review Letters 80.24 (1998), pp. 5325-5328 (cit. on p. 34).

[64] L. Sanchis et al.

"Reflectance properties of two-dimensional sonic band-gap crystals". In: J. Acoust. Soc. Am. 109.6 (2001), pp. 2598-2605 (cit. on p. 34).

[65] C. Croënne et al. "Band gaps in phononic crystals: Generation mechanisms and interaction effects".

In: AIP Advances 1.041401 (2011) (cit. on p. 34).

[66] R. Picó et al. "Spatial filtering of sound beams by sonic crystals". In: Applied Acoustics 73.4 (2012), pp. 302-306 (cit. on p. 34).

[67] Wu L. Y and L. W. Chen.

"An acoustic bending waveguide designed by graded sonic crystals". In: Journal of Applied Physics 110.114507 (2011) (cit. on p. 34).

[68] A. Cicek et al.

"Slow sound propagation in a sonic crystal linear waveguide".

In: Journal of Applied Physics 111.013522 (2012) (cit. on p. 34).

[69] S. Peng et al. "Acoustic far-field focusing effect for two-dimensional graded negative refractive-index sonic crystals".

In: Applied Physics Letters 96.263502 (2010) (cit. on p. 34). 
[70] V. Romero-García et al. "Evanescent modes and level repulsion stats in sonic stubbed waveguides". In: New J. Phys. 14 (2012), pp. 1-21 (cit. on p. 34).

[71] A. Cebrecos et al. "Formation of collimated sound beams by three-dimensional sonic crystals". In: J. Appl. Phys. 111.104910 (2012) (cit. on p. 34).

[72] I. Pérez-Arjona et al. "Theoretical prediction of the nondiffractive propagation of sonic waves through periodic acoustic media". In: Phy. Rev. B 75.014304 (2007) (cit. on p. 34).

[73] V. Romero-García et al. "Angular Band Gaps in Sonic Crystals: Evanescent Waves and Spatial Complex Dispersion Relation". In: J. Vib. Acoust. 135.041012 (2013) (cit. on p. 34).

[74] Acoustics. Sound scattering properties of surfaces. Part 2: Measurement of the directional diffusion coefficient in a free field. Standard. International Organization for Standardization, 2012 (cit. on pp. 37, 107).

[75] J. V. Sánchez-Pérez et al. "Acoustic barriers based on periodic arrays of scatterers". In: Applied Physics Letters 81 (2002) (cit. on pp. 38, 87).

[76] G. Campa and S. M. Camporeale. "Application of Transfer Matrix Method in Acoustics". In: Proceedings of the COMSOL Conference 2010 (cit. on pp. 46, 87).

[77] B. H. Song and J. S. Bolton.

"A transfer-matrix approach for estimating the characteristic impedance and wave numbers of limp and rigid porous materials". In: J. Acoust. Soc. Am. 107.3 (2000), pp. 1131-1152 (cit. on pp. 46, 69).

[78] I. Herrero-Durá et al. "Matrix formulation in Acoustics: the transfer matrix method". In: Modelling in Science Education and Learning 12.2 (2019) (cit. on pp. 47, 55, 60). 
[79] A. Merkel et al. "Control of acoustic absorption in one-dimensioal scattering by resonant scatterers".

In: Applied Physics Letters 107.244102 (2015) (cit. on pp. 50, 60, 63).

[80] A. Duclos, D. Lafarge, and V. Pagneux.

"Transmission of acoustic waves through 2D phononic crystals: visco-thermal and multiple scattering effects".

In: Eur. Phys. J. Appl. Phys. 45.11302 (2009) (cit. on p. 52).

[81] G. Theocharis et al. "Limits of slow sound propagation and transparency in lossy, locally resonant periodic structures". In: New Journal of Physics 16.093017 (2014) (cit. on p. 52).

[82] K. F. Graff. Wave motion in elastic solids. Oxford University Press, 1975 (cit. on p. 53).

[83] S. Timoshenko and S. Woinowsky-Krieger. Theory of plates and shells. 2nd. McGraw-Hill Book Company, 1987 (cit. on p. 53).

[84] D. Takahashi and M. Tanaka. "Flexural vibration of perforated plates and porous elastic materials under acoustic loading".

In: J. Acoust. Soc. Am. 112.4 (2002), pp. 1456-1464 (cit. on p. 53).

[85] E. Ventsel and T. Krauthammer.

Thin plates and shells. Theory, analysis and applications.

Marcel Dekker, Inc., 2001 (cit. on p. 53).

[86] V. V. Krylov and F. J. B. S. Tilman. "Acoustic 'black holes' for flexural waves as effective vibration dampers".

In: Journal of Sound and Vibration 204.3 (2004), pp. 605-619 (cit. on p. 53).

[87] M. D. Guild, V. M. García-Chocano, and J. Sánchez-Dehesa. "Aerogel as a soft acoustic metamaterial for airborne sound". In: Physical Review Applied 5.034012 (2016) (cit. on p. 53).

[88] D. H. Lee and Y. P. Kwon. "Estimation of the absorption of multiple layer perforated panel systems by transfer matrix method". 
In: Journal of Sound and Vibration 278 (2004), pp. 847-860 (cit. on p. 54).

[89] K. Sakagami, K. Matsutani, and M. Morimoto. "Sound absorption of a double-leaf micro-perforated panel with an air-back cavity and a rigid-back wall: detailed analysis with a Helmholtz-Kirchhoff integral formulation". In: Applied Acoustics 71 (2010), pp. 411-417 (cit. on p. 54).

[90] D. Li, D. Chang, and B. Liu. "Enhanced low- to mid- frequency sound absorption using parallel-arranged perforated plates with extended tubes and porous material".

In: Applied Acoustics 127 (2017), pp. 316-323 (cit. on p. 54).

[91] G. Wu et al. "Experimental and frequency-domain study of acoustic damping of single-layer perforated plates".

In: Aerospace Science and Technology 69 (2017), pp. 432-438 (cit. on p. 54).

[92] M. Farooqui, T. Elnady, and W. Akl. "Sound attenuation in ducts using locally resonant periodic aluminum patches".

In: J. Acoust. Soc. Am. 139.3277 (2017) (cit. on p. 54).

[93] Y. Y. Lee, E. W. M. Lee, and C. F. Ng. "Sound absorption of a finite flexible micro-perforated panel backed by an air cavity".

In: Journal of Sound and Vibration 287 (2004), pp. 227-243 (cit. on p. 56).

[94] A. W. Leissa. Vibration of plates. 2nd. 1969 (cit. on pp. 56, 57).

[95] L. Cremer, M. Heckl, and B. A. T. Petersson. Structural-borne sound. Structural vibrations and sound radiation at audio frequencies. 3rd. Springer, 2005 (cit. on pp. 57, 60).

[96] K. Sakagami et al. "Sound absorption of a cavity-backed membrane: A step towards design method for membrane-type absorbers".

In: Applied Acoustics 49.3 (1996), pp. 237-247 (cit. on p. 60). 
[97] E. E. Ungar, D. Ross, and E. M. Kerwin. Damping of flexural vibrations by alternate visco-elastic and elastic layers. Tech. rep. Wright Air Development Center, 1959 (cit. on p. 60).

[98] M. Cai, O. Painter, and K. J. Vahala. "Observation of critical coupling in a fiber taper to a silica-microsphere whispering-gallery mode system". In: Physics Review Letters 85 (2000) (cit. on p. 63).

[99] M. Molerón, M. Serra-Garcia, and C. Daraio. "Visco-thermal effects in acoustic metamaterials: from total transmission to total reflection and high absorption". In: New Journal of Physics 18 (2016) (cit. on p. 63).

[100] C. Meng et al. "Acoustic coherent perfect absorbers as sensitive null detectors". In: Scientific Reports 7.43574 (2017) (cit. on p. 64).

[101] R. C. Chanaud. "Effects of geometry on the resonance frequency of Helmholtz resonators".

In: Journal of Sound and Vibration 178.3 (1994), pp. 337-348 (cit. on p. 66).

[102] R. L. Panton and J. M. Miller. "Resonance frequencies of cylindrical Helmholtz resonators". In: J. Acoust. Soc. Am. 57.6 (1975), pp. 1533-1535 (cit. on p. 67).

[103] J. Kergomard and A. García. "Simple discontinuities in acoustic waveguides at low frequencies: critical analysis and formulae". In: Journal of Sound and Vibration 114.3 (1987), pp. 465-479 (cit. on p. 71).

[104] V. Dubos et al. "Theory of sound propagation in a duct with a branched tube using modal decompositon". In: Acustica 85 (1999), pp. 153-169 (cit. on p. 71).

[105] F. P. Mechel. Formulas of acoustics. 2nd ed. H. Springer-Verlag, Berlin (Springer Science \& Business Media), 2008 (cit. on p. 71). 
[106] M. R. Stinson. "The propagation of plane sound waves in narrow and wide circular tubes, and generalization to uniform tubes of arbitrary cross-sectional shape".

In: J. Acoust. Soc. Am. 89.2 (1991), pp. 550-558 (cit. on p. 71).

[107] P. E. Gill and E. Wong. Mixed Integer Nonlinear Programming. Sequential Quadratic Programming methods. Springer, 2011 (cit. on p. 76).

[108] Anycubic i3 Mega User Manual. Tech. rep. Anycubic (cit. on p. 78).

[109] Acoustics. Determination of sound absorption coefficient and impedance in impedances tubes. Part 2: Transfer-function method. Standard. International Organization for Standardization, 2002 (cit. on pp. 78, 79, 93, 103).

[110] J. Leng. "Controlling flexural waves using subwavelength perfect absorbers: application to acoustic black holes".

PhD thesis. Le Mans Université, 2019 (cit. on p. 88).

[111] VEGA C User's Manual. Tech. rep. Ariane Space, 2018 (cit. on pp. 88-90, 118).

[112] I. Herrero-Durà et al. "Modeling of continuous elastic systems by using the Finite Element Method". In: Modeling in Science Education and Learning 10.2 (2017), pp. 193-201 (cit. on p. 92).

[113] F. Ihlenburg. Finite Element Analysis of acoustics scattering. Springer, 1998 (cit. on p. 92).

[114] S. S. Rao. The Finite Element Method in Engineering. $5^{\text {th }}$ ed. (cit. on p. 92).

[115] K. A. Oladejo, R. Abu, and M. D. Adewale. "Effective modeling and simulation of engineering problems with COMSOL Multiphysics". In: International Journal of Science and Technology 10.2 (2012), pp. $742-748$ (cit. on p. 92). 
[116] J. Smardzewsi et al. "Sound absorption of wood-based materials". In: Holzforschung 69.4 (2015), pp. 431-439 (cit. on p. 108).

[117] S. Ausina-Sebastià. Caracterización de la cámara anecoica de la escuela universitaria de Gandia. Valencia, 1998 (cit. on p. 108).

[118] R. Lanoye et al. "Measuring the free field acoustic impedance and absorption coefficient of sound absorbing materials with a combined particle velocity-pressure sensor". In: J. Acoust. Soc. Am. 119.5 (2006), pp. 2826-2831 (cit. on p. 109).

[119] K. Hirosawa, K. Takashima, and H. Nakagawa.

"Comparison of three measurement techniques for the normal absorption coefficient of sound absorbing materials in the free field". In: J. Acoust. Soc. Am. 126.6 (2009), pp. 3020-3027 (cit. on p. 109).

[120] V. Achilleos, O. Richoux, and G. Theocharis. "Coherent perfect absorption induced by the nonlinearity of a Helmholtz resonator". In: J. Acoust. Soc. Am. 140.1 (2016), EL94-EL100 (cit. on p. 120).

[121] R. T. Beyer. "Nonlinear Acoustics". In: AJP. 41 (1973), pp. 1060-1067 (cit. on pp. 120, 121). 
Appendix 


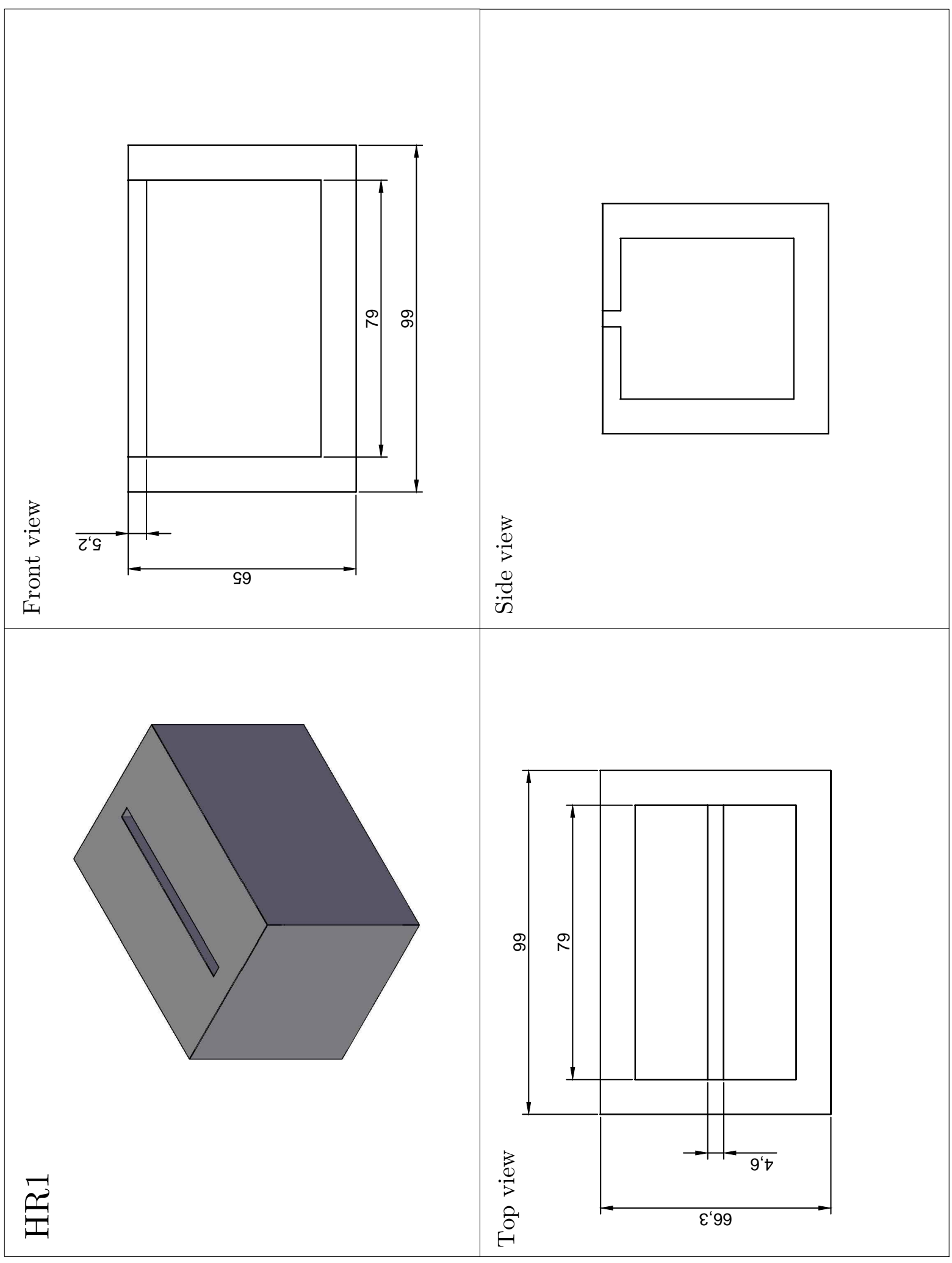




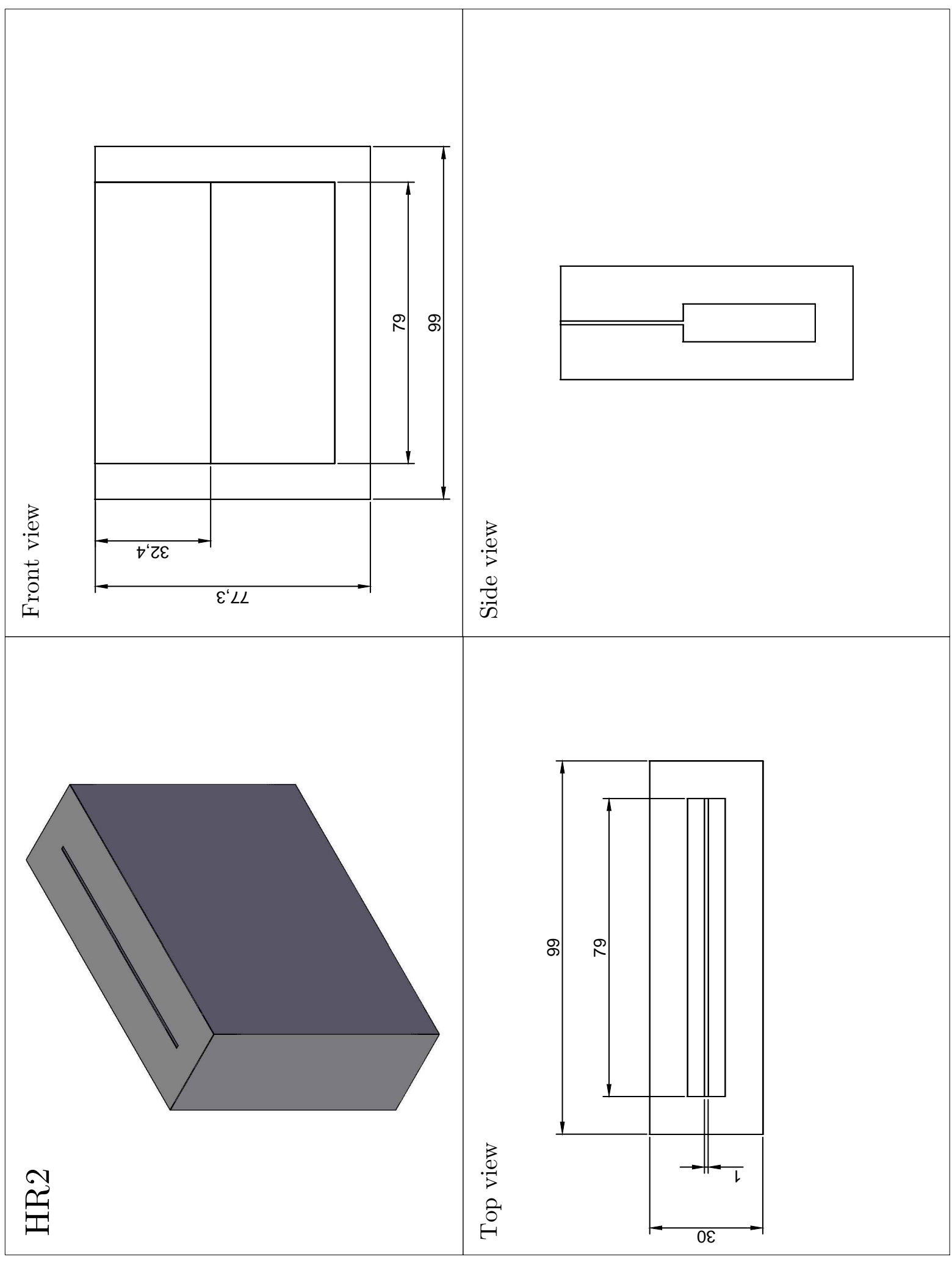



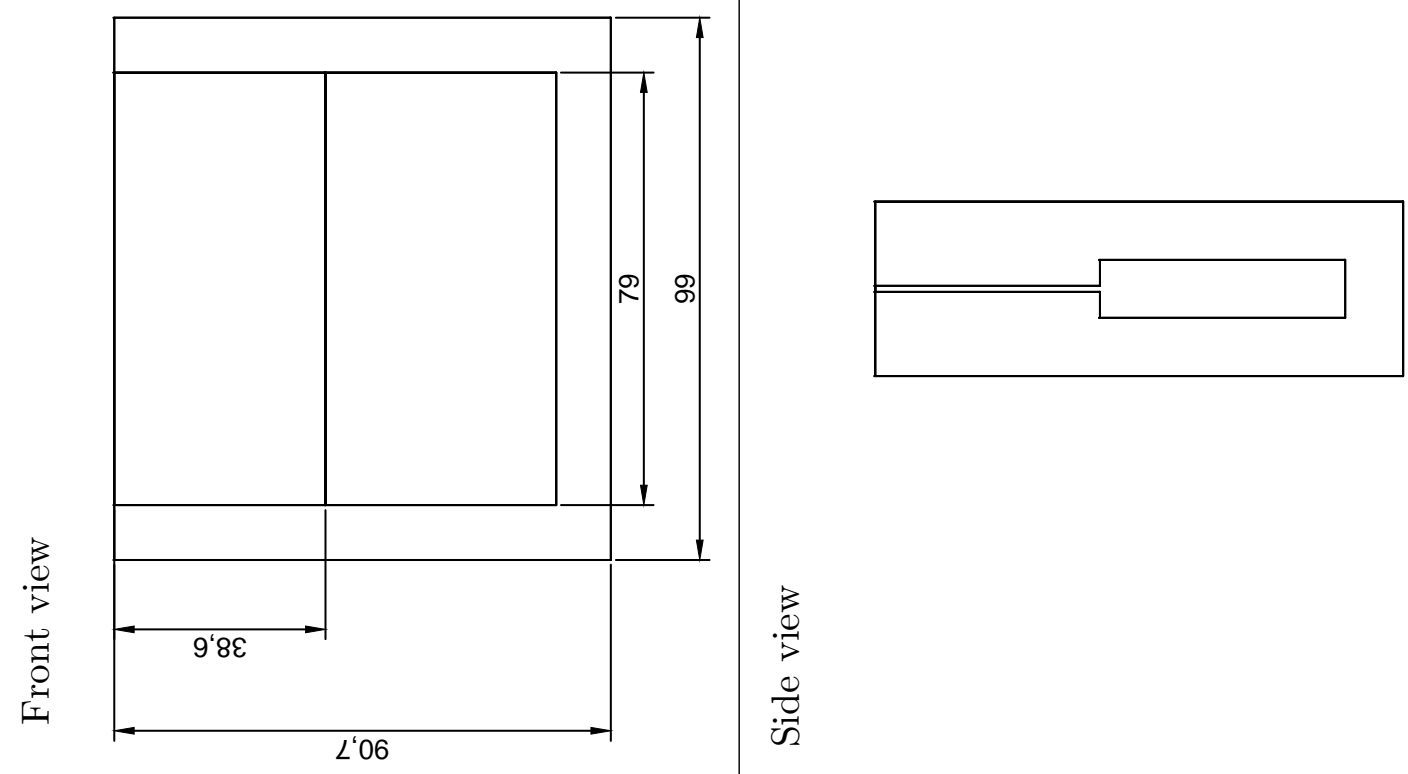

0
0
0
0
0
0
0

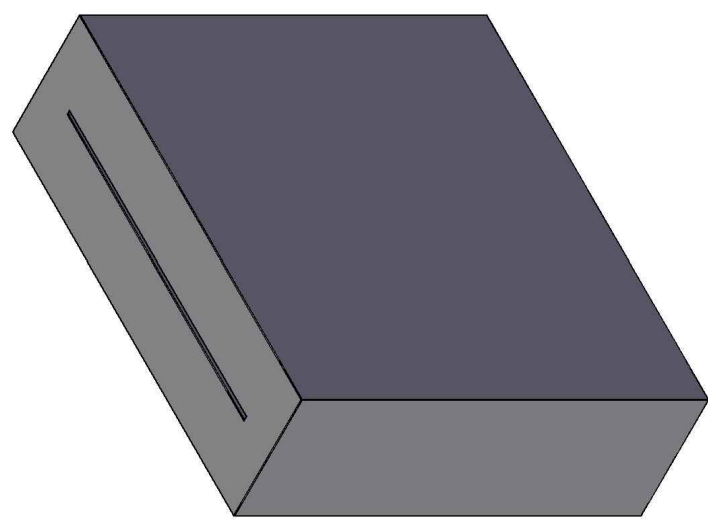

密

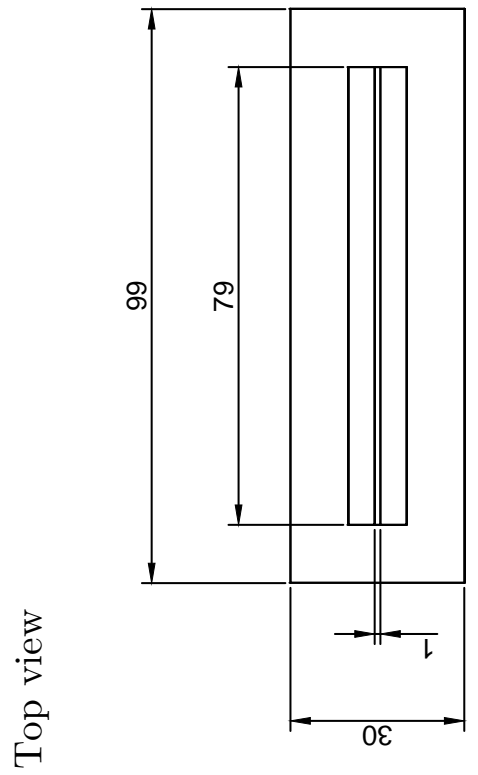




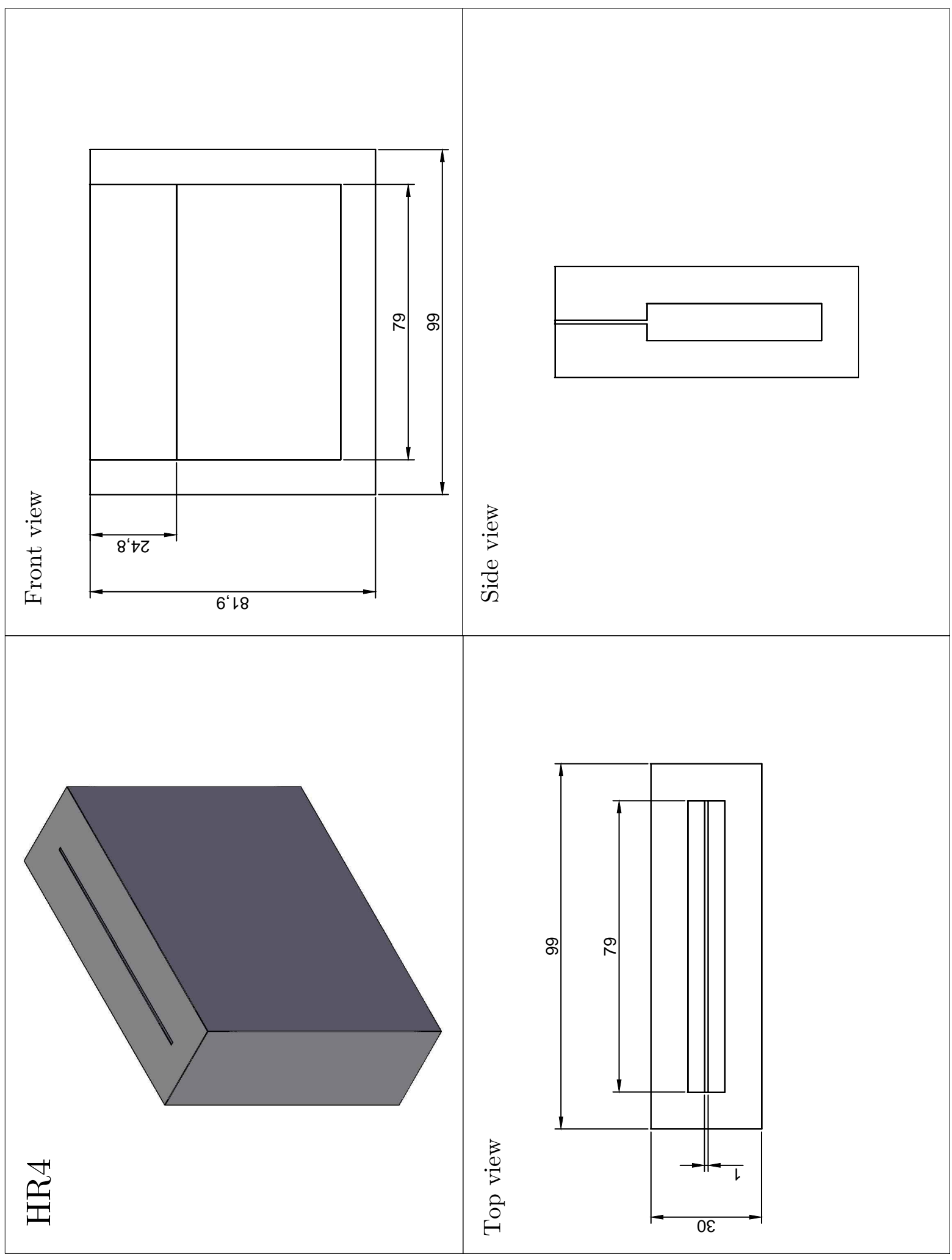




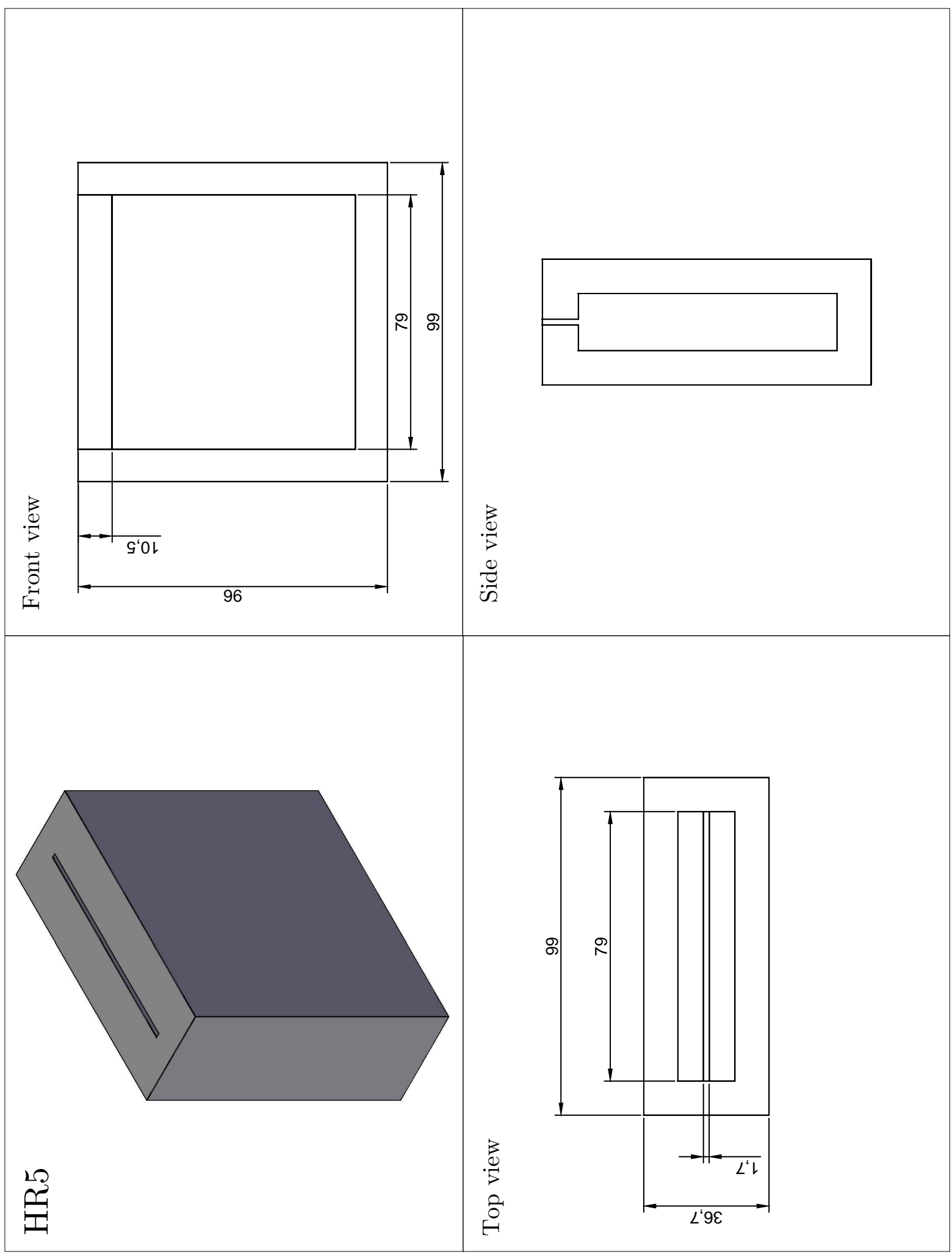

